\title{
Stereoselective $C$-Glycosylation Reactions of Ribose Derivatives: Electronic Effects of Five-Membered Ring Oxocarbenium Ions
}

\author{
Catharine H. Larsen, Brian H. Ridgway, Jared T. Shaw, Deborah M. Smith, and K. A. Woerpel* \\ Department of Chemistry, University of California \\ Irvine, CA 92697-2025
}

\section{Supporting Information}

\section{Contents:}
I. Synthesis of Tetrahydrofuran Acetates
$S-1$
II. Allyltrimethylsilane Addition to Tetrahydrofuran Acetates
$S-15$
III. Stereochemical proofs of allyl products
$S-19$
IV. Bibliography
$S-30$
V. Analytical Data
$S-32$

General. ${ }^{1} \mathrm{H}$ NMR and ${ }^{13} \mathrm{C}$ NMR spectra were recorded at ambient temperature at $500 \mathrm{MHz}$ and $125 \mathrm{MHz}$, respectively, using a Bruker DRX500 spectrometer. The data are reported as follows: chemical shift in ppm from internal tetramethylsilane on the $\square$ scale, multiplicity $(\mathrm{br}=$ broad, $\mathrm{s}=\operatorname{singlet}, \mathrm{d}=$ doublet, $\mathrm{t}=$ triplet, $\mathrm{q}=$ quartet, $\mathrm{m}=$ multiplet), coupling constants $(\mathrm{Hz})$, and integration. Infrared (IR) spectra were obtained using a MIDAC Prospect FT-IR spectrometer. High resolution mass spectra were acquired on a VG Analytical 7070E or Fisons Autospec spectrometers, and were obtained by peak matching. Microanalyses were performed by Atlantic Microlab, Atlanta, GA. Analytical gas-liquid chromatography (GC) analyses were performed on a Hewlett Packard 5890 Level 4 Chromatograph, equipped with a split-mode injection system and a flame ionization detector. A fused silica capillary column $(30 \mathrm{~m} \square 0.32 \mathrm{~mm}$ ) wall coated with DB-1 (J \& W Scientific) was used with helium as the carrier gas (16 psi column head pressure). Melting points are reported uncorrected. Liquid chromatography was performed using forced flow (flash chromatography) of the indicated solvent system on EM Reagents silica gel $\left(\mathrm{SiO}_{2}\right) 60$ (230-400 mesh). All reactions were carried out under an atmosphere of nitrogen in glassware that had been flame-dried under a stream of nitrogen. Unless otherwise noted, all reagents were commercially obtained and, where appropriate, purified prior to use. $\mathrm{THF}, \mathrm{Et}_{2} \mathrm{O}$, toluene, and $\mathrm{CH}_{2} \mathrm{Cl}_{2}$ were dried by filtration through alumina according to the procedure of Grubbs. ${ }^{1}$

The syntheses of compounds 8a, 9a, 14, 15, 18a, 19a, 20, 21, 22, 23, 24, 25, 26a-c, and 27a-c were described in the Supporting Information of a previous publication from our laboratory (J. Am. Chem. Soc. 1999, 121, 12208-12209.)

\section{Synthesis of Tetrahydrofuran Acetates}

A. 1-O-Acetyl-2-deoxy-2-fluoro-4,5-O,O-dibenzyl-D-arabinose (8b) Synthetic Scheme.

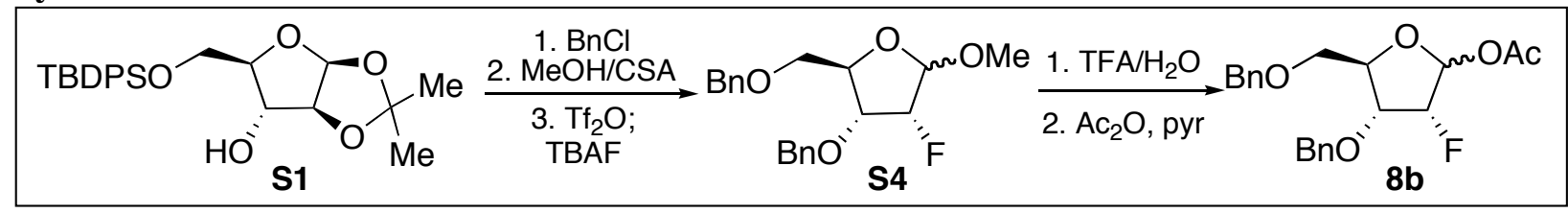




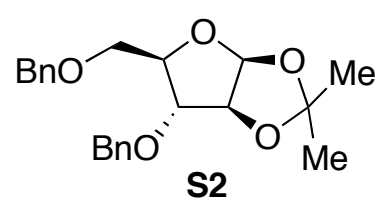

1,2- $\boldsymbol{O}, \boldsymbol{O}$-Isopropylidene-3,5-O,O-dibenzyl-D-arabinose (S2). A solution of $\mathbf{S 1}^{2}(0.981 \mathrm{~g}, 2.29 \mathrm{mmol})$ in 10 $\mathrm{mL}$ of THF was treated with benzyl chloride $(1.60 \mathrm{~mL}, 13.7 \mathrm{mmol})$ and powdered $\mathrm{KOH}(1.15 \mathrm{~g}, 20.6 \mathrm{mmol}$, freshly ground with a mortar and pestle). The reaction mixture was heated to reflux for $16 \mathrm{~h}$, during which time an insoluble gum that had formed became a free flowing slurry. After cooling the mixture to $22{ }^{\circ} \mathrm{C}$, the solution was filtered through a plug of glass wool. The resultant filtrate was washed with $3 \square 10 \mathrm{~mL}$ of THF and the washings were concentrated in vacuo. The excess benzyl chloride was removed by distillation (30 Torr) and the resultant oil was purified by flash chromatography (6:94 - 8:92 EtOAc/hexanes) to yield the product as a colorless oil $(0.602 \mathrm{~g}, 71 \%):{ }^{1} \mathrm{H}$ NMR $\left(500 \mathrm{MHz}, \mathrm{CDCl}_{3}\right) \square 7.31(\mathrm{~m}, 10 \mathrm{H}), 5.90(\mathrm{~d}, J=4.4,1 \mathrm{H}), 4.64(\mathrm{~d}, J=$ $4.0,1 \mathrm{H}), 4.57(\mathrm{~m}, 4 \mathrm{H}), 4.27(\mathrm{~m}, 1 \mathrm{H}), 4.03(\mathrm{~d}, J=3.1,1 \mathrm{H}), 3.63(\mathrm{~d}, J=6.0,2 \mathrm{H}), 1.44(\mathrm{~s}, 3 \mathrm{H}), 1.32(\mathrm{~s}, 3 \mathrm{H}) ;{ }^{13} \mathrm{C}$ NMR $\left(125 \mathrm{MHz}, \mathrm{CDCl}_{3}\right) \square 138.0,137.3,128.5,128.3,127.9,127.8,127.7,127.6,112.7,105.7,85.1,83.5$, 83.0, 73.3, 71.6, 70.0, 27.0, 26.3; IR (thin film) 2937, 1453, 1374, 1098, $1027 \mathrm{~cm}^{-1}$; HRMS (CI/isobutane) $\mathrm{m} / z$ calcd for $\mathrm{C}_{22} \mathrm{H}_{26} \mathrm{O}_{5}(\mathrm{M})^{+}$370.1780, found 370.1780. Anal. Calcd for $\mathrm{C}_{22} \mathrm{H}_{26} \mathrm{O}_{5}: \mathrm{C}, 71.33 ; \mathrm{H}, 7.07$. Found: C, 71.07; H, 7.13.

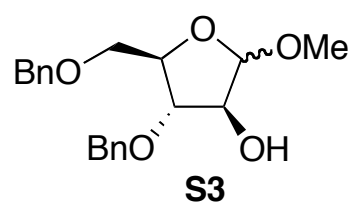

1-O-Methyl-3,5-O,O-dibenzyl-D-arabinose (S3). A solution of $\mathbf{S 2}(0.548 \mathrm{~g}, 1.48 \mathrm{mmol})$ in $20 \mathrm{~mL}$ of $\mathrm{MeOH}$ was treated with a small amount $(\leq 10 \mathrm{mg})$ of $d$,l-camphorsulfonic acid, then heated to reflux for $12 \mathrm{~h}$. The reaction mixture was cooled to $22{ }^{\circ} \mathrm{C}$, treated with 3 drops of $\mathrm{Et}_{3} \mathrm{~N}$, and concentrated in vacuo to produce a yellow oil. ${ }^{1} \mathrm{H}$ NMR spectroscopic analysis of the unpurified product showed a pair of diastereomers in a ratio of 60:40. Purification by flash chromatography (20:80 - 30:70 EtOAc/hexanes) provided the product as a colorless oil $(0.491 \mathrm{~g}, 96 \%)$. The purified product was characterized as a mixture of diastereomers: ${ }^{1} \mathrm{H}$ NMR $\left(500 \mathrm{MHz}, \mathrm{CDCl}_{3}\right) \square 7.30(\mathrm{~m}, 10 \mathrm{H}), 4.90(\mathrm{~s}, 0.6 \mathrm{H}), 4.86(\mathrm{~d}, J=4.8,0.4 \mathrm{H}), 4.59(\mathrm{~m}, 4 \mathrm{H}), 4.25(\mathrm{~m}, 1 \mathrm{H}), 4.13(\mathrm{~m}$, $1 \mathrm{H}), 3.84(\mathrm{~m}, 1 \mathrm{H}), 3.64(\mathrm{~m}, 0.6 \mathrm{H}), 3.53(\mathrm{~d}, J=5.6,0.8 \mathrm{H}), 3.41(\mathrm{~s}, 3 \mathrm{H} ; \mathrm{m}, 0.6 \mathrm{H}), 3.32(\mathrm{~d}, J=10.7,0.6 \mathrm{H}), 2.58$ $(\mathrm{d}, J=9.6,0.4 \mathrm{H}) ;{ }^{13} \mathrm{C}$ NMR $\left(125 \mathrm{MHz}, \mathrm{CDCl}_{3}\right) \square 138.0,137.9,137.7,136.9,128.5,128.4,128.3,128.0,127.9$, $127.8,127.7,110.5,102.6,84.8,84.6,83.6,80.7,78.0,77.8,73.7,73.3,72.1,72.0,71.8,69.7,55.4,55.3$; IR (thin film) 3445, 2912, 1454, 1103, 1047, 1028, 738, $699 \mathrm{~cm}^{-1}$; HRMS (CI/isobutane) $\mathrm{m} / \mathrm{z}$ calcd for $\mathrm{C}_{20} \mathrm{H}_{25} \mathrm{O}_{5}$ $(\mathrm{M}+\mathrm{H})^{+}$345.1702, found 345.1705. Anal. Calcd for $\mathrm{C}_{20} \mathrm{H}_{24} \mathrm{O}_{5}: \mathrm{C}, 69.75 ; \mathrm{H}, 6.95$. Found: C, 69.47; H, 6.95.

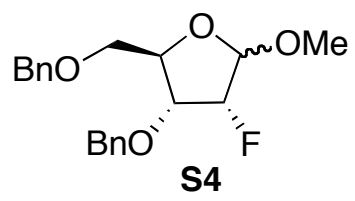

1-O-Methyl-2-deoxy-2-fluoro-4,5-O,O-dibenzyl-D-arabinose (S4). A solution of $\mathbf{S 3}$ (2.29 g, $6.65 \mathrm{mmol})$ in $100 \mathrm{~mL}$ of $\mathrm{CH}_{2} \mathrm{Cl}_{2}$ was cooled to $0{ }^{\circ} \mathrm{C}$, then treated with $5 \mathrm{~mL}$ of pyridine followed by trifluoromethanesulfonic anhydride $^{3}(1.45 \mathrm{~mL}, 8.64 \mathrm{mmol})$. After $20 \mathrm{~min}$, starting material was not visible by TLC and the reaction mixture was treated with $10 \mathrm{~mL}$ of $\mathrm{H}_{2} \mathrm{O}$. The layers were separated and the organic layer was concentrated in vacuo. The recovered material was dried on high vacuum line $(\leq 0.1$ Torr) for $10 \mathrm{~min}$, then dissolved in $50 \mathrm{~mL}$ 
of THF and cooled to $0{ }^{\circ} \mathrm{C}$. A solution of TBAF $(33 \mathrm{~mL}, 33 \mathrm{mmol}, 1.0 \mathrm{M}$ solution in THF) was added and the reaction mixture was warmed to $22{ }^{\circ} \mathrm{C}$ overnight, then concentrated in vacuo to afford a dark red oil. ${ }^{1} \mathrm{H}$ NMR spectroscopic analysis of the unpurified product showed a single epimer at the acetal center. Purification by flash chromatography (10:90 EtOAc/toluene) provided the product as a colorless oil $(0.682 \mathrm{~g}, 30 \%)$ : $[\square]_{25}^{\mathrm{D}} 1.4$, $[\square]^{405}{ }_{25} 0.3\left(c 1.15, \mathrm{CHCl}_{3}\right) ;{ }^{1} \mathrm{H}$ NMR $\left(500 \mathrm{MHz}, \mathrm{CDCl}_{3}\right) \square 7.30(\mathrm{~m}, 10 \mathrm{H}), 5.01(\mathrm{~d}, J=10.5,1 \mathrm{H}), 4.81-4.61(\mathrm{~m}$, $5 \mathrm{H}), 4.30(\mathrm{~m}, 1 \mathrm{H}), 4.07(\mathrm{~m}, 1 \mathrm{H}), 3.64(\mathrm{~m}, 1 \mathrm{H}), 3.54(\mathrm{~m}, 1 \mathrm{H}), 3.32(\mathrm{~s}, 3 \mathrm{H}) ;{ }^{13} \mathrm{C} \mathrm{NMR}\left(125 \mathrm{MHz}, \mathrm{CDCl}_{3}\right) \square$ 138.2, 137.3, 128.5, 128.3, 128.0, 127.9, 127.6, 105.6 (d, $J=29), 91.0$ (d, $J=185), 80.0,77.7$ (d, $J=16), 73.2$, 72.7, 70.9, 55.0; ${ }^{19} \mathrm{~F}$ NMR (376 MHz, $\mathrm{CDCl}_{3}$, fluorobenzene external standard) $\square-209.4$ (ddd, $J=53.2,24.6$, 10.6); IR (thin film) 2926, 1454, 1363, 1110, $737 \mathrm{~cm}^{-1}$. Anal. Calcd for $\mathrm{C}_{20} \mathrm{H}_{23} \mathrm{FO}_{4}$ : C, 69.35; H, 6.69. Found: C, $69.28 ; \mathrm{H}, 6.63$.

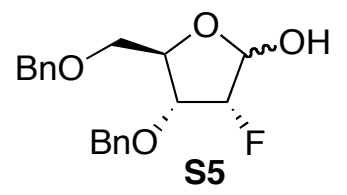

2-Deoxy-2-fluoro-4,5-O,O-dibenzyl-D-arabinose (S5). A sample of $\mathbf{S 4}(0.651 \mathrm{~g}, 1.88 \mathrm{mmol})$ was treated with $10 \mathrm{~mL}$ of $90: 10$ trifluoroacetic acid/ $\mathrm{H}_{2} \mathrm{O}$ and was stirred for $5 \mathrm{~h}$. The reaction mixture was partitioned between $\mathrm{H}_{2} \mathrm{O}$ and $\mathrm{CH}_{2} \mathrm{Cl}_{2}$, then treated with $2 \mathrm{~mL}$ portions of aqueous $10 \mathrm{M} \mathrm{NaOH}$ until the aqueous layer was determined by $\mathrm{pH}$ paper to be neutral. The organic layer was recovered and the aqueous layer was extracted with $2 \square 20 \mathrm{~mL}$ of $\mathrm{CH}_{2} \mathrm{Cl}_{2}$. The combined organic layers were dried $\left(\mathrm{Na}_{2} \mathrm{SO}_{4}\right)$ and concentrated in vacuo to provide a brown oil. The acetal product was determined to be an $84: 16$ mixture of diastereomers by ${ }^{13} \mathrm{C}$ NMR spectroscopic analysis. Purification by flash chromatography (10:90 - 14:86 - 18:82 EtOAc/hexanes) provided the product as a colorless oil $(0.325 \mathrm{~g}, 54 \%)$. The purified product was characterized as a mixture of diastereomers: ${ }^{1} \mathrm{H}$ NMR $\left(500 \mathrm{MHz}, \mathrm{CDCl}_{3}\right) \square 7.29(\mathrm{~m}, 10 \mathrm{H}), 5.33(\mathrm{~m}, 1 \mathrm{H}), 4.93(\mathrm{dt}, J=51.2,4.3,0.2 \mathrm{H}), 4.82$ $(\mathrm{d}, J=3.6,0.4 \mathrm{H}), 4.76-4.25(\mathrm{~m}, 6 \mathrm{H}), 4.07(\mathrm{~m}, 0.2 \mathrm{H}), 3.94(\mathrm{~m}, 0.2 \mathrm{H}), 3.67(\mathrm{~m}, 1.5 \mathrm{H}), 3.50(\mathrm{~m}, 1.5 \mathrm{H}) ;{ }^{13} \mathrm{C} \mathrm{NMR}$ $\left(125 \mathrm{MHz}, \mathrm{CDCl}_{3}\right) \square 137.7,137.2,136.99,136.95,128.53,128.49,128.4,128.12,128.05,128.0,127.9,127.84$, 127.77, 127.6, 99.7 (d, $J=29), 95.7$ (d, $J=19), 92.0(\mathrm{~d}, J=188), 88.4$ (d, $J=198), 80.7$ (d, $J=3.5), 80.5,76.7$, $76.3(\mathrm{~d}, J=16), 73.5,73.10,73.09,72.7,69.4,68.9 ;{ }^{19} \mathrm{~F} \mathrm{NMR}\left(376 \mathrm{MHz}, \mathrm{CDCl}_{3}\right.$, fluorobenzene external standard) प-206.74 (m), -206.89 (m); IR (thin film) 3415 (br, s) 2926, 2867, 1454, 1070, $744 \mathrm{~cm}^{-1}$; HRMS (CI/isobutane) $\mathrm{m} / \mathrm{z}$ calcd for $\mathrm{C}_{19} \mathrm{H}_{20} \mathrm{O}_{4} \mathrm{~F}(\mathrm{M}-\mathrm{H})^{+} 331.1345$, found 331.1342. Anal. Calcd for $\mathrm{C}_{19} \mathrm{H}_{21} \mathrm{O}_{4} \mathrm{~F}: \mathrm{C}$, 68.66; H, 6.37. Found: C, 68.67; H, 6.39.

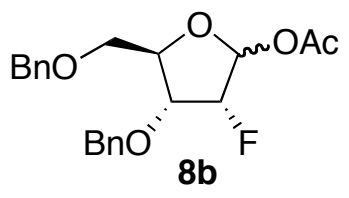

1-O-Acetyl-2-deoxy-2-fluoro-4,5-O,O-dibenzyl-D-arabinose (8b). A solution of $\mathbf{S 5}(0.297 \mathrm{~g}, 0.894 \mathrm{mmol})$ in $5 \mathrm{~mL}$ of pyridine was treated with $2 \mathrm{~mL}$ of $\mathrm{Ac}_{2} \mathrm{O}$ and DMAP $(\leq 5 \mathrm{mg})$, then stirred at $22{ }^{\circ} \mathrm{C}$ for $16 \mathrm{~h}$. The reaction mixture was concentrated in vacuo and partitioned between $20 \mathrm{~mL}$ each of EtOAc and $\mathrm{H}_{2} \mathrm{O}$. The organic layer was washed with $2 \square 10 \mathrm{~mL}$ of saturated aqueous $\mathrm{Na}_{2} \mathrm{HPO}_{4}$, dried $\left(\mathrm{Na}_{2} \mathrm{SO}_{4}\right)$, and concentrated in vacuo to provide a brown oil. ${ }^{1} \mathrm{H}$ NMR spectroscopic analysis of the unpurified product showed a single epimer at the acetal center. Purification by flash chromatography (10:90 EtOAc/hexanes) provided the product as a

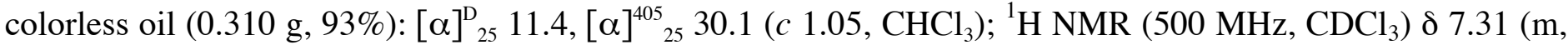


$10 \mathrm{H}), 6.23(\mathrm{~d}, J=10.2,1 \mathrm{H}), 4.85(\mathrm{dd}, J=52.6,3.6,1 \mathrm{H}), 4.70(\mathrm{~d}, J=11.7,1 \mathrm{H}), 4.55(\mathrm{~m}, 3 \mathrm{H}), 4.31(\mathrm{~m}, 1 \mathrm{H})$, $4.25(\mathrm{ddd}, J=24.1,7.9,3.6,1 \mathrm{H}), 3.71(\mathrm{dd}, J=11.1,2.9,1 \mathrm{H}), 3.59(\mathrm{dd}, J=11.1,3.9,1 \mathrm{H}), 1.91(\mathrm{~s}, 3 \mathrm{H}) ;{ }^{13} \mathrm{C}$ NMR $\left(125 \mathrm{MHz}, \mathrm{CDCl}_{3}\right) \square$ 169.0, 138.1, 137.2, 128.5, 128.3, 128.1, 127.8, 127.6, 127.4, $97.7(\mathrm{~d}, J=32), 90.8$ (d, $J=188), 81.1,76.2(\mathrm{~d}, J=16), 73.2,72.8,69.0,20.9 ;{ }^{19} \mathrm{~F} \mathrm{NMR}\left(376 \mathrm{MHz}, \mathrm{CDCl}_{3}\right.$, fluorobenzene external standard) -209.1 (ddd, $J=52.6,24.0,10.3$ ); IR (thin film) 2866, 1748, 1223, 1099, $961 \mathrm{~cm}^{-1}$; HRMS (CI/isobutane) $m / z$ calcd for $\mathrm{C}_{21} \mathrm{H}_{22} \mathrm{FO}_{5}(\mathrm{M}-\mathrm{H})^{+}, 373.1452$, found 373.1453. Anal. Calcd for $\mathrm{C}_{21} \mathrm{H}_{23} \mathrm{FO}_{5}: \mathrm{C}$, 67.37; H, 6.19. Found: C, 67.27; H, 6.16.

\section{B. $\left(2 R^{*}\right)$-1-Acetoxy-2-benzyloxytetrahydrofuran (10) Synthetic Scheme.}
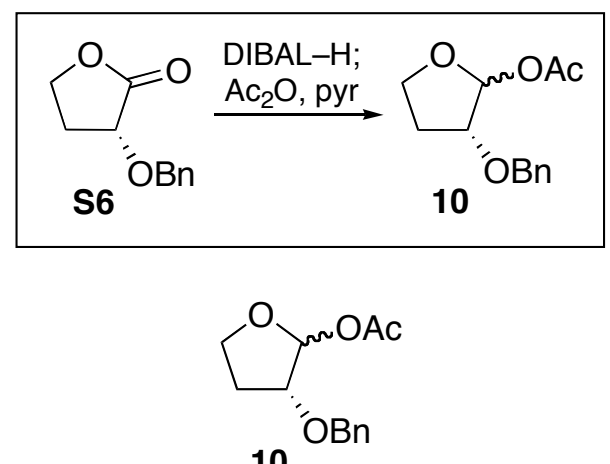

General Procedure for Reduction and Acylation of $\square$-Lactones: ${ }^{4}$ To a cooled $\left(-78{ }^{\circ} \mathrm{C}\right)$ solution of $\square$ lactone in $\mathrm{CH}_{2} \mathrm{Cl}_{2}(0.10-0.20 \mathrm{M})$ was added DIBAL-H (1.2 equiv, $1.5 \mathrm{M}$ in toluene). After the mixture was stirred at $-78{ }^{\circ} \mathrm{C}$ for $2 \mathrm{~h}$, pyridine (4.0 equiv) and DMAP (1.2 equiv) were added, followed by the dropwise addition of $\mathrm{Ac}_{2} \mathrm{O}$ (5.0 equiv). After the reaction mixture was allowed to warm to $22{ }^{\circ} \mathrm{C}$ over $12 \mathrm{~h}, 5 \mathrm{~mL}$ of saturated aqueous $\mathrm{NH}_{4} \mathrm{Cl}$ was added and the reaction mixture was concentrated in vacuo. The remaining oil was dissolved in MTBE. The organic layer was washed with $4 \square 10 \mathrm{~mL}$ of saturated aqueous $\mathrm{Na}_{2} \mathrm{HPO}_{4}, 4 \square 10 \mathrm{~mL}$ of saturated aqueous $\mathrm{NaH}_{2} \mathrm{PO}_{4}$, and $5 \square 10 \mathrm{~mL}$ of saturated aqueous $\mathrm{CuSO}_{4}$. The organic phase was dried $\left(\mathrm{Na}_{2} \mathrm{SO}_{4}\right)$ and concentrated in vacuo to provide a dark orange residue.

$\left(2 R^{*}\right)$-1-Acetoxy-2-benzyloxytetrahydrofuran (10). The standard reductive acylation procedure was followed with lactone $\mathbf{S 6}^{5}(0.302 \mathrm{~g}, 1.57 \mathrm{mmol})$ in $16 \mathrm{~mL}$ of $\mathrm{CH}_{2} \mathrm{Cl}_{2}$, with DIBAL-H $(1.25 \mathrm{~mL}, 1.88 \mathrm{mmol})$, pyridine $(0.508 \mathrm{~mL}, 6.28 \mathrm{mmol})$, DMAP $(0.231 \mathrm{~g}, 1.88 \mathrm{mmol})$, and $\mathrm{Ac}_{2} \mathrm{O}(0.742 \mathrm{~mL}, 7.85 \mathrm{mmol})$. After $24 \mathrm{~h}$ at $22{ }^{\circ} \mathrm{C}$, additional pyridine $(0.508 \mathrm{~mL}, 6.28 \mathrm{mmol})$ and $\mathrm{Ac}_{2} \mathrm{O}(0.742 \mathrm{~mL}, 7.85 \mathrm{mmol})$ were added and the solution was stirred for $24 \mathrm{~h} .{ }^{1} \mathrm{H}$ NMR spectroscopic analysis of the unpurified product showed a pair of diastereomers in a ratio of 75:25. Purification by flash chromatography (10:90 - 30:70 EtOAc/hexanes) yielded acetate $\mathbf{1 0}$ as a colorless oil $(0.244 \mathrm{~g}, 66 \%)$. The purified product was characterized as a mixture of diastereomers: ${ }^{1} \mathrm{H}$ NMR $\left(500 \mathrm{MHz}, \mathrm{CDCl}_{3}\right) \square 7.36-7.26(\mathrm{~m}, 5 \mathrm{H}), 6.26(\mathrm{~d}, J=3.9,1 \mathrm{H}), 4.59(\mathrm{~m}, 2 \mathrm{H}), 4.12(\mathrm{~m}, 2.25 \mathrm{H}), 3.88(\mathrm{dd}, J=$ $16.3,8.7,0.75 \mathrm{H}), 2.35(\mathrm{~m}, 1 \mathrm{H}), 2.09(\mathrm{~s}, 2.25 \mathrm{H}), 2.06(\mathrm{~m}, 1 \mathrm{H}), 2.03(\mathrm{~s}, 0.75 \mathrm{H}) ;{ }^{13} \mathrm{C} \mathrm{NMR}\left(125 \mathrm{MHz}, \mathrm{CDCl}_{3}\right) \square$ 170.3, 169.9, 137.5, 128.4, 128.3, 127.8, 127.7, 127.64, 127.58, 100.2, 94.1, 82.0, 78.3, 72.7, 71.4, 68.4, 66.2, 29.6, 27.9, 21.2, 21.1; IR (thin film) $3088,2989,1744 \mathrm{~cm}^{-1}$; HRMS (Cl/isobutane) $\mathrm{m} / \mathrm{z}$ calcd for $\mathrm{C}_{11} \mathrm{H}_{13} \mathrm{O}_{2}$ (M $\left.-\mathrm{C}_{2} \mathrm{H}_{3} \mathrm{O}_{2}\right)^{+}$177.0916, found 177.0911. Anal. Calcd for $\mathrm{C}_{13} \mathrm{H}_{16} \mathrm{O}_{4}:$ C, 66.09; H, 6.83. Found: C, 66.10; H, 6.83.

C. $\left(3 S^{*}, 4 R^{*}\right)$-1-Acetoxy-3-benzyloxy-4-(3-phenyl)propyltetrahydrofuran (12) Synthetic Scheme. 

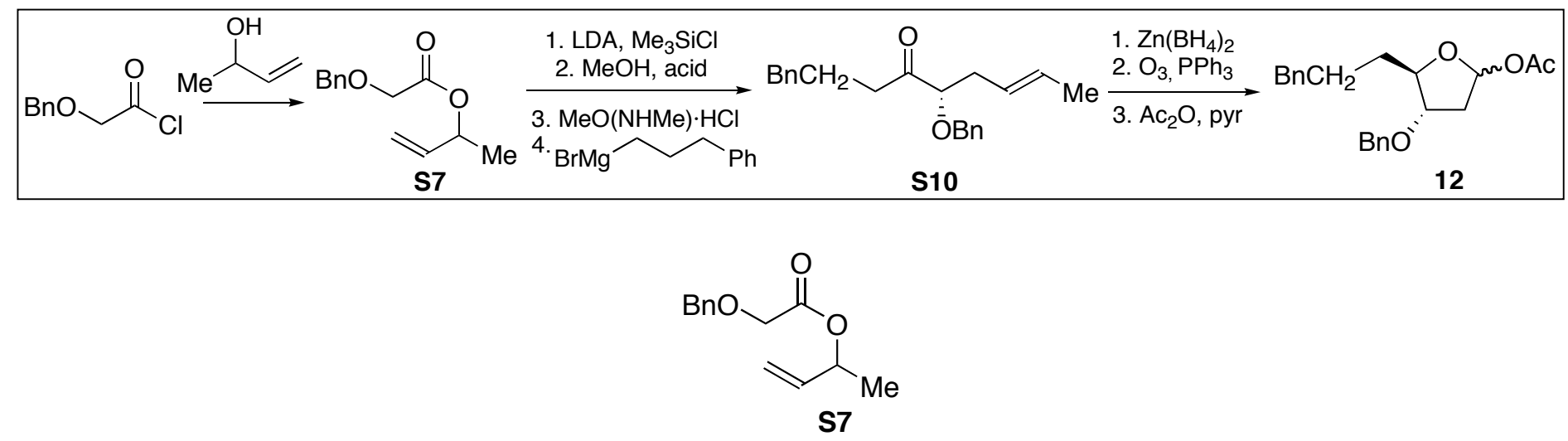

3-Buten-1-yl-(2'-benzyloxy)acetate (S7). To a cooled $\left(0{ }^{\circ} \mathrm{C}\right)$ solution of 3-buten-2-ol $(0.762 \mathrm{~g}, 10.6 \mathrm{mmol})$ in $20 \mathrm{~mL}$ of $\mathrm{CH}_{2} \mathrm{Cl}_{2}$ was added pyridine $(1.71 \mathrm{~mL}, 21.1 \mathrm{mmol})$ followed by benzyloxyacetyl chloride $(2.00 \mathrm{~mL}$, $12.7 \mathrm{mmol}){ }^{6}$ After $24 \mathrm{~h}$ at $22{ }^{\circ} \mathrm{C}$, the reaction mixture was treated with $0.5 \mathrm{M} \mathrm{HCl}$ and the organic layer was recovered. The aqueous layer was extracted with $3 \square 10 \mathrm{~mL}$ of $\mathrm{CH}_{2} \mathrm{Cl}_{2}$. The combined organic layers were dried $\left(\mathrm{Na}_{2} \mathrm{SO}_{4}\right)$ and concentrated in vacuo to provide a pale yellow oil. Purification by flash chromatography (4:96 - 5:95 EtOAc/hexanes) provided the product as a colorless oil $(2.09 \mathrm{~g}, 90 \%):{ }^{1} \mathrm{H} \mathrm{NMR}\left(500 \mathrm{MHz} \mathrm{CDCl}_{3}\right)$ $\square 7.38(\mathrm{~m}, 5 \mathrm{H}), 5.85(\mathrm{~m}, 1 \mathrm{H}), 5.46(\mathrm{~m}, 1 \mathrm{H}), 5.27(\mathrm{~d}, J=17.3,1 \mathrm{H}), 5.16(\mathrm{~d}, J=10.9,1 \mathrm{H}), 4.64(\mathrm{~s}, 2 \mathrm{H}), 4.09(\mathrm{~s}$, 2H), $1.35(\mathrm{~d}, J=6.5,3 \mathrm{H}) ;{ }^{13} \mathrm{C}$ NMR $\left(125 \mathrm{MHz}, \mathrm{CDCl}_{3}\right) \square$ 169.6, 137.2, 137.1, 128.4, 128.03, 127.96, 116.3, 73.3, 71.7, 67.3, 19.9; IR (thin film) 2983, 2934, 1752, $1131 \mathrm{~cm}^{-1} ; \mathrm{HRMS}\left(\mathrm{CI} / \mathrm{NH}_{3}\right) \mathrm{m} / z$ calcd for $\mathrm{C}_{13} \mathrm{H}_{20} \mathrm{NO}_{3}$ $\left(\mathrm{M}+\mathrm{NH}_{4}\right)^{+}$238.1443, found 238.1445. Anal. Calcd for $\mathrm{C}_{13} \mathrm{H}_{16} \mathrm{O}_{3}: \mathrm{C}, 70.89 ; \mathrm{H}, 7.32$. Found: $\mathrm{C}, 70.72 ; \mathrm{H}$, 7.25 .

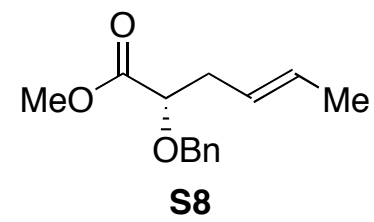

Methyl 2-benzyloxy-4-hexenoate (S8). To a cooled $\left(0^{\circ} \mathrm{C}\right)$ solution of $i$ - $\mathrm{Pr}_{2} \mathrm{NH}(1.32 \mathrm{~mL}, 9.42 \mathrm{mmol})$ in $\mathrm{THF}$ $(35 \mathrm{~mL})$ was added $n$-BuLi $\left(2.9 \mathrm{~mL}, 7.1 \mathrm{mmol} ; 2.4 \mathrm{M}\right.$ in hexanes). The reaction mixture was cooled to $-78^{\circ} \mathrm{C}$ and a solution of $\mathbf{S 7}(1.04 \mathrm{~g}, 4.71 \mathrm{mmol})$ in $10 \mathrm{~mL}$ of THF was added by cannula. ${ }^{7}$ After $40 \mathrm{~min}$ at $-78^{\circ} \mathrm{C}$, the reaction mixture was treated with $1.5 \mathrm{~mL}$ of a $1: 1$ mixture of $\mathrm{Et}_{3} \mathrm{~N} / \mathrm{Me}_{3} \mathrm{SiCl}$ (prepared by admixture and centrifugation for $10 \mathrm{~min}$ to produce a clear supernatant solution and white precipitate). After $12 \mathrm{~h}$ at $22^{\circ} \mathrm{C}$, the solution was treated with $100 \mathrm{~mL}$ of $5 \mathrm{M} \mathrm{NaOH}$ and was stirred for an additional $10 \mathrm{~min}$. The mixture was extracted with $2 \square 20 \mathrm{~mL}$ of $\mathrm{Et}_{2} \mathrm{O}$. The aqueous layer was cooled to $0{ }^{\circ} \mathrm{C}$ and treated with $2 \mathrm{~mL}$ portions of 12

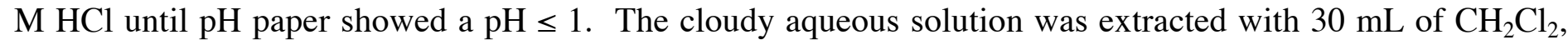
followed by $8 \square 10 \mathrm{~mL}$ of $\mathrm{CH}_{2} \mathrm{Cl}_{2}$. The combined organic layers were dried $\left(\mathrm{Na}_{2} \mathrm{SO}_{4}\right)$ and concentrated in vacuo to provide the product as a colorless oil $(0.826 \mathrm{~g}, 80 \%):{ }^{1} \mathrm{H} \mathrm{NMR}\left(500 \mathrm{MHz}, \mathrm{CDCl}_{3}\right) \square 9.48(\mathrm{br} \mathrm{s}, 1 \mathrm{H})$, $7.36(\mathrm{~m}, 5 \mathrm{H}), 5.55(\mathrm{~m}, 1 \mathrm{H}), 5.47(\mathrm{~m}, 1 \mathrm{H}), 4.73(\mathrm{~d}, J=11.7,1 \mathrm{H}), 4.50(\mathrm{~d}, J=11.7,1 \mathrm{H}), 4.00(\mathrm{dd}, J=6.8,5.1$, $1 \mathrm{H}), 2.52(\mathrm{~m}, 2 \mathrm{H}), 1.67(\mathrm{dd}, J=6.3,1.1,3 \mathrm{H}) ;{ }^{13} \mathrm{C} \mathrm{NMR}\left(125 \mathrm{MHz}, \mathrm{CDCl}_{3}\right) \square 176.6,137.1,129.1,128.4,128.0$, 124.9, 77.7, 72.3, 35.7, 18.0. This material was converted to the methyl ester for purification and full characterization. A sample of the carboxylic acid $(0.455 \mathrm{~g}, 2.07 \mathrm{mmol})$ was dissolved in $50 \mathrm{~mL}$ of $\mathrm{MeOH}$ and treated with $1 \mathrm{~mL}$ of $\mathrm{H}_{2} \mathrm{SO}_{4}$. After $12 \mathrm{~h}$ at $22{ }^{\circ} \mathrm{C}$, the reaction mixture was neutralized with $50 \mathrm{~mL}$ of saturated aqueous $\mathrm{NaH}_{2} \mathrm{PO}_{4}$. The mixture was concentrated in vacuo and the resulting solution was extracted with $3 \square 30$ 
$\mathrm{mL}$ of $\mathrm{CH}_{2} \mathrm{Cl}_{2}$. The combined organic layers were dried $\left(\mathrm{Na}_{2} \mathrm{SO}_{4}\right)$ and concentrated in vacuo to afford $\mathbf{S 8}$ as a colorless oil. Purification by flash chromatography (4:96 EtOAc/hexanes) provided the product as a colorless oil $(0.484 \mathrm{~g}, 100 \%):{ }^{1} \mathrm{H}$ NMR $\left(500 \mathrm{MHz}, \mathrm{CDCl}_{3}\right) \square 7.43(\mathrm{~m}, 5 \mathrm{H}), 5.53(\mathrm{~m}, 1 \mathrm{H}), 5.44(\mathrm{~m}, 1 \mathrm{H}), 4.70(\mathrm{~d}, J=11.8$, $1 \mathrm{H}), 4.44(\mathrm{~d}, J=11.8,1 \mathrm{H}), 3.96(\mathrm{t}, J=6.3,1 \mathrm{H}), 3.74(\mathrm{~s}, 3 \mathrm{H}), 2.46(\mathrm{~m}, 2 \mathrm{H}), 1.66(\mathrm{dt}, J=6.5,1.2,3 \mathrm{H}) ;{ }^{13} \mathrm{C}$ NMR $\left(125 \mathrm{MHz}, \mathrm{CDCl}_{3}\right) \square 172.8,137.5,128.7,128.3,127.9,127.8,125.3,78.2,72.2,51.8,36.2,18.0$; IR (thin film) $3030,2951,1752,1454,1436,1129 \mathrm{~cm}^{-1}$; HRMS (CI/isobutane) $\mathrm{m} / z$ calcd for $\mathrm{C}_{14} \mathrm{H}_{19} \mathrm{O}_{3}(\mathrm{M}+\mathrm{H})^{+}$ 235.1334, found 235.1345. Anal. Calcd for $\mathrm{C}_{14} \mathrm{H}_{18} \mathrm{O}_{3}$ : C, 71.77; H, 7.74. Found: C, 71.84; H, 7.74.

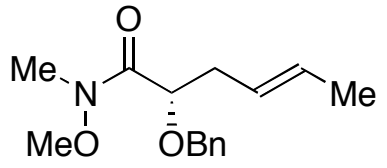

S9

$\mathrm{N}$-methyl- $\mathrm{N}$-methoxy-2-benzyloxy-4-hexenamide (S9). A slurry of $\mathrm{N}, \mathrm{O}$-methoxymethylamine hydrochloride $(1.193 \mathrm{~g}, 12.23 \mathrm{mmol})$ in $10 \mathrm{~mL}$ of $\mathrm{CH}_{2} \mathrm{Cl}_{2}$ was cooled to $0{ }^{\circ} \mathrm{C}$ and treated with a solution of $\mathrm{Me}_{3} \mathrm{Al}(6.1 \mathrm{~mL}, 12$ $\mathrm{mmol}$; $2.0 \mathrm{M}$ in toluene) resulting in mild effervescence. After $10 \mathrm{~min}$, a solution of $\mathbf{S 8}(0.573 \mathrm{~g}, 2.45 \mathrm{mmol})$ in $10 \mathrm{~mL}$ of $\mathrm{CH}_{2} \mathrm{Cl}_{2}$ was added by cannula. ${ }^{8}$ After $12 \mathrm{~h}$ at $22{ }^{\circ} \mathrm{C}$, the reaction mixture was poured into saturated aqueous sodium potassium tartrate and the aqueous layer was extracted with $5 \square 20 \mathrm{~mL}$ of $\mathrm{CH}_{2} \mathrm{Cl}_{2}$. The combined organic layers were dried $\left(\mathrm{Na}_{2} \mathrm{SO}_{4}\right)$ and concentrated in vacuo to provide a colorless oil. Purification by flash chromatography $(20: 80 \mathrm{EtOAc} /$ hexanes $)$ provided the product as a colorless oil $(0.483 \mathrm{~g}, 75 \%):{ }^{1} \mathrm{H}$ $\operatorname{NMR}\left(500 \mathrm{MHz}, \mathrm{CDCl}_{3}\right) \square 7.35(\mathrm{~m}, 5 \mathrm{H}), 5.5(\mathrm{~m}, 2 \mathrm{H}), 4.69(\mathrm{~d}, J=12.1,1 \mathrm{H}), 4.42(\mathrm{~d}, J=12.1,1 \mathrm{H}), 4.31(\mathrm{~s}$, $1 \mathrm{H}), 3.56(\mathrm{~s}, 3 \mathrm{H}), 3.20(\mathrm{~s}, 3 \mathrm{H}), 2.44(\mathrm{~m}, 2 \mathrm{H}), 1.66(\mathrm{~d}, J=5.8,3 \mathrm{H}) ;{ }^{13} \mathrm{C} \mathrm{NMR}\left(125 \mathrm{MHz}, \mathrm{CDCl}_{3}\right) \square 172.8,137.8$, 128.3, 128.2, 127.9, 127.6, 126.1, 75.5, 71.3, 61.3, 35.5, 32.3, 18.0; IR (thin film) 2936, 1673, 1454, $1109 \mathrm{~cm}^{-1}$; HRMS $\left(\mathrm{CI} / \mathrm{NH}_{3}\right) \mathrm{m} / z$ calcd for $\mathrm{C}_{15} \mathrm{H}_{22} \mathrm{NO}_{3}(\mathrm{M}+\mathrm{H})^{+} 264.1599$, found 264.1604. Anal. Calcd for $\mathrm{C}_{15} \mathrm{H}_{21} \mathrm{NO}_{3}$ : C, 68.42; H, 8.04; N, 5.32. Found: C, 68.15; H, 7.97; N, 5.23.

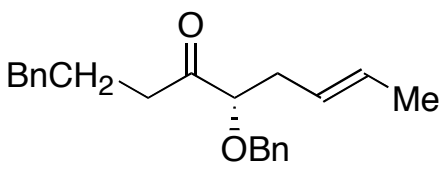

S10

1-Phenyl-5-benzyloxy-7-nonen-4-one (S10). A slurry of magnesium turnings $(0.147 \mathrm{~g}, 6.04 \mathrm{mmol})$ in $10 \mathrm{~mL}$ of THF was treated with a few crystals of iodine and several drops of 1-phenyl-3-bromopropane. The mixture was heated to reflux until the color of iodine disappeared. A solution of 1-phenyl-3-bromopropane $(0.834 \mathrm{~mL}$, $5.49 \mathrm{mmol}$ ) in $10 \mathrm{~mL}$ of THF was added dropwise over $10 \mathrm{~min}$ to the magnesium slurry while heating to maintain reflux, then cooled to $22^{\circ} \mathrm{C}$. The solution of Grignard reagent was added by cannula to a cooled $(-78$ $\left.{ }^{\circ} \mathrm{C}\right)$ solution of amide $\mathbf{S 9}(0.483 \mathrm{~g}, 1.83 \mathrm{mmol}){ }^{9}$ After the solution was stirred for $1.5 \mathrm{~h}$ at $0{ }^{\circ} \mathrm{C}$, the reaction mixture was added to a 1:1 mixture of ice/EtOH $(60 \mathrm{~mL})$ and subsequently treated with $2 \mathrm{~mL}$ of concentrated $\mathrm{H}_{2} \mathrm{SO}_{4}$. The mixture was extracted with $5 \square 20 \mathrm{~mL}$ of $\mathrm{CH}_{2} \mathrm{Cl}_{2}$. The combined organic layers were dried $\left(\mathrm{Na}_{2} \mathrm{SO}_{4}\right)$ and concentrated in vacuo to provide a colorless oil. Purification by flash chromatography (3:97 EtOAc/hexanes) provided $\mathbf{S 1 0}$ as a colorless oil $(0.589 \mathrm{~g}, 100 \%):{ }^{1} \mathrm{H}$ NMR $\left(500 \mathrm{MHz}, \mathrm{CDCl}_{3}\right) \square 7.30(\mathrm{~m}, 7 \mathrm{H})$, $7.18(\mathrm{~m}, 3 \mathrm{H}), 5.49(\mathrm{~m}, 1 \mathrm{H}), 5.38(\mathrm{~m}, 1 \mathrm{H}), 4.52(\mathrm{~d}, J=11.7,1 \mathrm{H}), 4.44(\mathrm{~d}, J=11.7,1 \mathrm{H}), 3.80(\mathrm{t}, J=6.6,1 \mathrm{H})$, $2.60(\mathrm{~m}, 2 \mathrm{H}), 2.53(\mathrm{~m}, 2 \mathrm{H}), 2.37(\mathrm{~m}, 2 \mathrm{H}), 1.89(\mathrm{~m}, 2 \mathrm{H}), 1.64(\mathrm{dd}, J=6.3,1.3,3 \mathrm{H}) ;{ }^{13} \mathrm{C}$ NMR $(125 \mathrm{MHz}$, $\left.\mathrm{CDCl}_{3}\right) \square 212.2$, 141.6, 137.5, 128.6, 128.44, 128.35, 127.9, 127.8, 125.9, 125.3, 84.7, 72.3, 37.4, 35.4, 35.1, 
24.5, 18.0; IR (thin film) 2935, 1714, 1496, 1454, $1095 \mathrm{~cm}^{-1}$; HRMS $\left(\mathrm{CI} / \mathrm{NH}_{3}\right) \mathrm{m} / z$ calcd for $\mathrm{C}_{22} \mathrm{H}_{27} \mathrm{O}_{2}(\mathrm{M}+$ H) ${ }^{+}$323.2011, found 323.2022. Anal. Calcd for $\mathrm{C}_{22} \mathrm{H}_{26} \mathrm{O}_{2}: \mathrm{C}, 81.95 ; \mathrm{H}, 8.13$. Found: C, 81.86; H, 8.11.

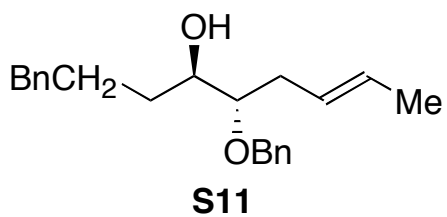

$\left(4 S^{*}, 5 R^{*}\right)$-1-Phenyl-5-benzyloxy-7-nonen-4-ol (S11). To a cooled $\left(-35^{\circ} \mathrm{C}\right)$ solution of ketone $\mathbf{S 1 0}(0.514 \mathrm{~g}$, $1.59 \mathrm{mmol})$ in $30 \mathrm{~mL}$ of $\mathrm{Et}_{2} \mathrm{O}$ was added $\mathrm{Zn}\left(\mathrm{BH}_{4}\right)_{2}\left(9.9 \mathrm{~mL}, 6.4 \mathrm{mmol}, 0.69 \mathrm{M}\right.$ solution in $\left.\mathrm{Et}_{2} \mathrm{O}^{10}\right)$. After 30 min, the mixture was treated with $50 \mathrm{~mL}$ of $0.5 \mathrm{M} \mathrm{NaOH}$ and $50 \mathrm{~mL}$ of $\mathrm{CH}_{2} \mathrm{Cl}_{2}{ }^{11}$ Saturated aqueous sodium potassium tartrate was added to disrupt the resultant emulsion, then the organic layer was recovered and the aqueous layer was extracted with $3 \square 20 \mathrm{~mL}$ of $\mathrm{CH}_{2} \mathrm{Cl}_{2}$. The combined organic layers were dried $\left(\mathrm{Na}_{2} \mathrm{SO}_{4}\right)$ and concentrated in vacuo to provide a colorless oil. GC analysis of the unpurified product showed a single diastereomer. Purification by flash chromatography (4:96 EtOAc/hexanes) provided $\mathbf{S 1 1}$ as a colorless oil (0.304 g, 59\%): ${ }^{1} \mathrm{H}$ NMR $\left(500 \mathrm{MHz}, \mathrm{CDCl}_{3}\right) \square 7.31(\mathrm{~m}, 7 \mathrm{H}), 7.18(\mathrm{~m}, 3 \mathrm{H}), 5.48(\mathrm{~m}, 2 \mathrm{H}), 4.59(\mathrm{~d}, J=11.6,1 \mathrm{H})$, $4.52(\mathrm{~d}, J=11.6,1 \mathrm{H}), 3.75(\mathrm{~m}, 1 \mathrm{H}), 3.36(\mathrm{~m}, 1 \mathrm{H}), 2.63(\mathrm{t}, J=7.7,2 \mathrm{H}), 2.32(\mathrm{~m}, 1 \mathrm{H}), 2.19(\mathrm{~m}, 1 \mathrm{H}), 1.97(\mathrm{~d}, J=$ $4.8,1 \mathrm{H}), 1.88(\mathrm{~m}, 1 \mathrm{H}), 1.66(\mathrm{~d}, J=5.9,3 \mathrm{H} ; \mathrm{m}, 1 \mathrm{H}), 1.50(\mathrm{~m}, 2 \mathrm{H}) ;{ }^{13} \mathrm{C}$ NMR $\left(125 \mathrm{MHz}, \mathrm{CDCl}_{3}\right) \square 142.3,138.4$, $128.40,128.37,128.3,127.8,127.7,127.4,125.5,82.1,72.0,71.9,35.8,32.4,31.6,27.8,18.0$; IR (thin film) 3452, 2934, 1496, 1453, $1089 \mathrm{~cm}^{-1}$; HRMS $\left(\mathrm{CI} / \mathrm{NH}_{3}\right) \mathrm{m} / \mathrm{z}$ calcd for $\mathrm{C}_{22} \mathrm{H}_{29} \mathrm{O}_{2}(\mathrm{M}+\mathrm{H})^{+} 325.2168$, found 325.2167. Anal. Calcd for $\mathrm{C}_{22} \mathrm{H}_{28} \mathrm{O}_{2}$ : C, 81.44; $\mathrm{H}, 8.70$. Found: $\mathrm{C}, 81.15 ; \mathrm{H}, 8.60$.

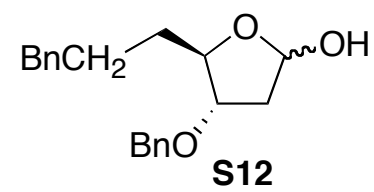

$\left(3 S^{*}, 4 R^{*}\right)$-3-Benzyloxy-1-hydroxy-4-(3-phenyl)propyltetrahydrofuran (S12). Ozone was bubbled through a cooled $\left(-78^{\circ} \mathrm{C}\right)$ solution of $\mathbf{S 1 1}(0.100 \mathrm{~g}, 0.308 \mathrm{mmol})$ in $10 \mathrm{~mL}$ of $\mathrm{CH}_{2} \mathrm{Cl}_{2}$ for $10 \mathrm{~min}$ to yield a persistent blue color. The solution was treated with $\mathrm{PPh}_{3}(0.243 \mathrm{~g}, 0.925 \mathrm{mmol})$ and was warmed to $22{ }^{\circ} \mathrm{C}$. The solvent was removed in vacuo, and the resultant white solid was triturated in $2 \mathrm{~mL}$ of MTBE, then treated with $8 \mathrm{~mL}$ of hexanes. The supernatant solution was removed by pipette and filtered through glass wool. The remaining white solid was twice treated with $10 \mathrm{~mL}$ of 20:80 MTBE/hexanes and the supernatant solutions were each filtered and combined with the previous washing. The combined solutions were concentrated in vacuo to yield a colorless oil and a small amount of white solid. ${ }^{1} \mathrm{H}$ NMR spectroscopic analysis of the unpurified product showed a pair of diastereomers in a ratio of 65:35. Purification by flash chromatography (12:88 - 16:84 EtOAc/hexanes) provided $\mathbf{S 1 2}$ as a colorless oil $(0.079 \mathrm{~g}, 82 \%)$. The purified product was characterized as a mixture of diastereomers: ${ }^{1} \mathrm{H}$ NMR $\left(500 \mathrm{MHz}, \mathrm{CDCl}_{3}\right) \square 7.35-7.16(\mathrm{~m}, 15 \mathrm{H}), 5.57(\mathrm{dd}, J=3.2,1.8,0.5 \mathrm{H}), 5.41$ $(\mathrm{dd}, J=10.3,4.7,1 \mathrm{H}), 4.54(\mathrm{~d}, J=11.9,1 \mathrm{H}), 4.51(\mathrm{~d}, J=11.9,1 \mathrm{H}), 4.50(\mathrm{~d}, J=11.8,0.5 \mathrm{H}), 4.45(\mathrm{~d}, J=11.8$, $0.5 \mathrm{H}), 4.34(\mathrm{~m}, 1 \mathrm{H}), 3.99(\mathrm{~m}, 1 \mathrm{H}), 3.81(\mathrm{~m}, 1 \mathrm{H}), 3.60(\mathrm{~m}, 1 \mathrm{H}), 2.92(\mathrm{~m}, 0.5 \mathrm{H}), 2.64(\mathrm{~m}, 3 \mathrm{H}), 2.14(\mathrm{~m}, 1.5 \mathrm{H})$, $2.03(\mathrm{~m}, 1.5 \mathrm{H}), 1.77-1.61(\mathrm{~m}, 4 \mathrm{H}), 1.42(\mathrm{~m}, 2 \mathrm{H}) ;{ }^{13} \mathrm{C} \mathrm{NMR}\left(125 \mathrm{MHz}, \mathrm{CDCl}_{3}\right) \square$ 142.2, 142.0, 136.9, 137.3, $128.5,128.4,128.29,128.25,127.9,127.7,125.8,125.7,98.8,98.5,83.7,83.3,82.2,82.0,71.6,71.3,39.9$, 38.7, 35.6, 35.0, 33.5, 27.7, 27.4; IR (thin film) 3412, 2938, 1496, 1454, $1086 \mathrm{~cm}^{-1}$; HRMS (EI) $\mathrm{m} / \mathrm{z}$ calcd for $\mathrm{C}_{20} \mathrm{H}_{22} \mathrm{O}_{2}\left(\mathrm{M}-\mathrm{H}_{2} \mathrm{O}\right)^{+}$294.1620, found 294.1629. Anal. Calcd for $\mathrm{C}_{20} \mathrm{H}_{24} \mathrm{O}_{3}: \mathrm{C}, 76.89 ; \mathrm{H}, 7.74$. Found: C, 76.59; H, 7.71 . 


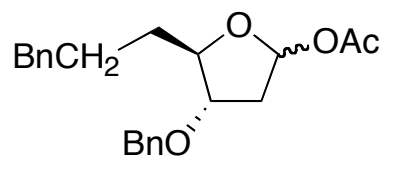

12

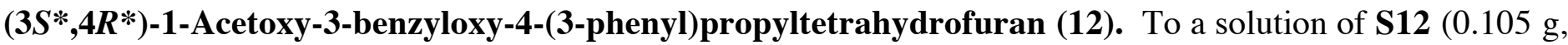
$0.336 \mathrm{mmol})$ in $8 \mathrm{~mL}$ of pyridine was added $2 \mathrm{~mL}$ of $\mathrm{Ac}_{2} \mathrm{O}$ and DMAP $(\leq 5 \mathrm{mg})$. The reaction mixture was stirred at $22{ }^{\circ} \mathrm{C}$ for $12 \mathrm{~h}$, then concentrated in vacuo and dried on a high vacuum line $(\leq 0.1 \mathrm{Torr})$ for $1 \mathrm{~h}$. ${ }^{1} \mathrm{H}$ NMR spectroscopic analysis of the unpurified product showed a pair of diastereomers in a ratio of 56:44. Purification by flash chromatography (6:94 - 8:92 EtOAc/hexanes) provided 12 as a colorless oil (0.108 g, $91 \%)$. The purified product was characterized as a mixture of diastereomers: ${ }^{1} \mathrm{H}$ NMR $\left(500 \mathrm{MHz}, \mathrm{CDCl}_{3}\right) \square$ (major isomer, distinctive peaks) $6.32(\mathrm{dd}, J=5.1,2.1,1 \mathrm{H}), 1.96(\mathrm{~s}, 3 \mathrm{H})$; (minor isomer, distinctive peaks) 6.26 $(\mathrm{m}, 1 \mathrm{H}), 2.06(\mathrm{~s}, 3 \mathrm{H})$; (both isomers) $7.32(\mathrm{~m}, 7 \mathrm{H}), 7.16(\mathrm{~m}, 3 \mathrm{H}), 4.48(\mathrm{~m}, 2 \mathrm{H}), 4.22(\mathrm{~m}, 0.5 \mathrm{H}), 4.11(\mathrm{~m}, 0.5 \mathrm{H})$, $3.98(\mathrm{~m}, 0.5 \mathrm{H}), 3.78(\mathrm{~m}, 0.5 \mathrm{H}), 2.64(\mathrm{~m}, 2 \mathrm{H}), 2.28(\mathrm{~m}, 1.5 \mathrm{H}), 2.13(\mathrm{~m}, 0.5 \mathrm{H}), 1.68(\mathrm{~m}, 4 \mathrm{H}) ;{ }^{13} \mathrm{C}$ NMR $(125$ $\left.\mathrm{MHz}, \mathrm{CDCl}_{3}\right) \square 170.6,170.1,142.03,141.97,137.8,137.7,128.41,128.37,128.2,127.8,127.7,127.6,125.7$, $98.5,98.2,84.8,84.5,81.4,81.1,71.7,71.5,38.6,37.9,35.6,35.5,34.3,33.1,27.3,27.2,21.4,21.3$; IR (thin film) 2939, 1742, 1237, $1110 \mathrm{~cm}^{-1}$; HRMS (FAB) $m / z$ calcd for $\mathrm{C}_{22} \mathrm{H}_{26} \mathrm{O}_{4} \mathrm{Na}(\mathrm{M}+\mathrm{Na})^{+} 377.1729$, found 377.1724. Anal. Calcd for $\mathrm{C}_{22} \mathrm{H}_{26} \mathrm{O}_{4}$ : C, 74.55; H, 7.35. Found: C, 74.43; H, 7.39.

\section{1-Acetoxy-3-benzyloxy-4,4-bis-benzyloxymethyltetrahydrofuran (16) Synthetic Scheme.}
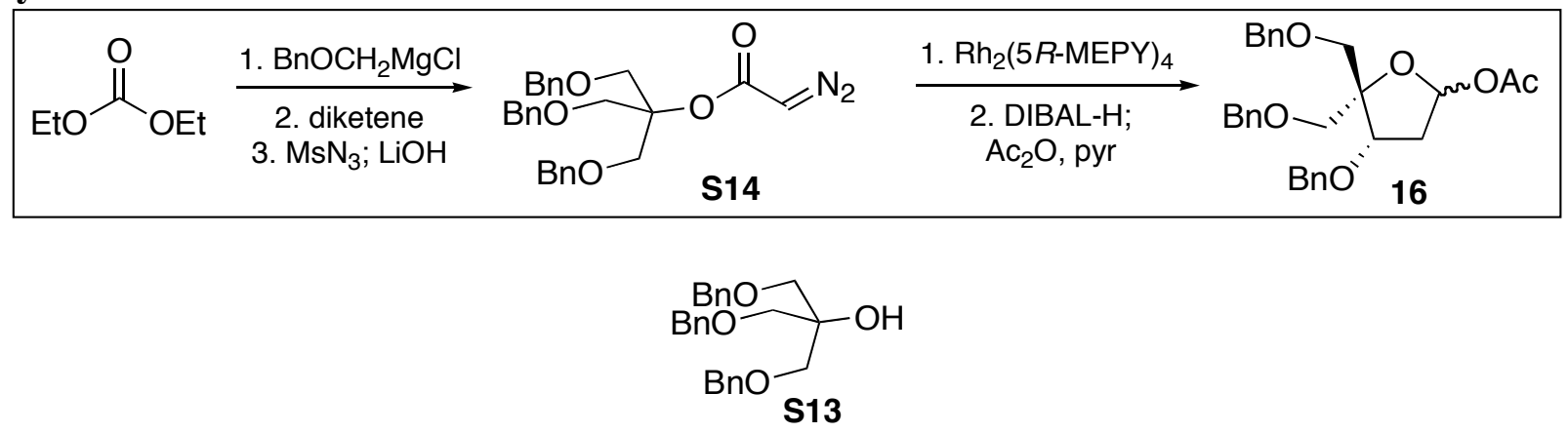

tris(Benzyloxymethyl)carbinol (S13). ${ }^{12}$ A slurry of magnesium turnings $(0.700 \mathrm{~g}, 28.8 \mathrm{mmol})$ in $3 \mathrm{~mL}$ of THF was treated with $\mathrm{HgCl}_{2}(\leq 5 \mathrm{mg})$, several crystals of iodine, and 3 drops of $\mathrm{BOMCl}$. The mixture was heated to reflux until the color of the iodine disappeared, then cooled to $0{ }^{\circ} \mathrm{C}$. A solution of BOMCl $(3.40 \mathrm{~mL}, 24.7$ $\mathrm{mmol}$ ) in $10 \mathrm{~mL}$ of THF was added dropwise over $30 \mathrm{~min}$, and the mixture was stirred for an additional $30 \mathrm{~min}$. The reaction mixture was cooled to $-30^{\circ} \mathrm{C}$, and a solution of diethyl carbonate $(0.750 \mathrm{~mL}, 6.17 \mathrm{mmol}$; distilled from $\left.\mathrm{CaH}_{2}\right)$ in $5 \mathrm{~mL}$ of THF was added dropwise. After $24 \mathrm{~h}$ at $22^{\circ} \mathrm{C}$, saturated aqueous $\mathrm{NH}_{4} \mathrm{Cl}(20 \mathrm{~mL})$ was added dropwise to the reaction mixture, which was stirred for an additional $30 \mathrm{~min}$. The mixture was treated with $10 \mathrm{~mL}$ of $\mathrm{CH}_{2} \mathrm{Cl}_{2}$, then the organic layer was recovered and the aqueous layer was extracted with $5 \square 10$ $\mathrm{mL}$ of $\mathrm{CH}_{2} \mathrm{Cl}_{2}$. The combined organic layers were dried $\left(\mathrm{Na}_{2} \mathrm{SO}_{4}\right)$ and concentrated in vacuo to provide a colorless oil. Purification by flash chromatography (6:94 - 8:92 - 10:90 EtOAc/hexanes) provided S13 as a colorless oil (1.10 g, 37\%): ${ }^{1} \mathrm{H}$ NMR (500 MHz, $\left.\mathrm{CDCl}_{3}\right) \square 7.30$ (m, 15H), $4.54(\mathrm{~s}, 6 \mathrm{H}), 3.55$ (s, $\left.6 \mathrm{H}\right), 2.72$ (s, $1 \mathrm{H}) ;{ }^{13} \mathrm{C}$ NMR $\left(125 \mathrm{MHz}, \mathrm{CDCl}_{3}\right) \square 138.2,128.3,127.6,74.1,73.5,71.1$; IR (thin film) 3456, 2862, 1454,1097 $\mathrm{cm}^{-1}$; HRMS (CI/isobutane) $m / z$ calcd for $\mathrm{C}_{25} \mathrm{H}_{29} \mathrm{O}_{4}(\mathrm{M}+\mathrm{H})^{+} 393.2065$, found 393.2069. Anal. Calcd for $\mathrm{C}_{25} \mathrm{H}_{28} \mathrm{O}_{4}$ : C, 75.76; H, 7.42. Found: C, 75.67; H, 7.36. 


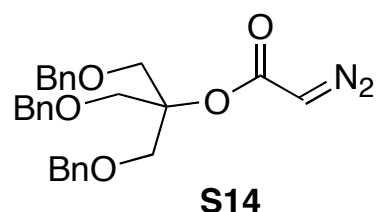

tris(Benzyloxymethyl)methyl diazoacetate (S14). To a cooled $\left(-20^{\circ} \mathrm{C}\right)$ solution of $\mathbf{S 1 3}(0.799 \mathrm{~g}, 2.04 \mathrm{mmol})$ in $50 \mathrm{~mL}$ of $\mathrm{Et}_{2} \mathrm{O}$ and DMAP $(\leq 10 \mathrm{mg})$ was added diketene $(0.204 \mathrm{~mL}, 2.65 \mathrm{mmol}){ }^{13}$ After $2.5 \mathrm{~h}$ at $22{ }^{\circ} \mathrm{C}$, the reaction mixture was treated with $2 \mathrm{~mL}$ of $\mathrm{MeOH}$, then concentrated in vacuo and dried on a high vacuum line. The acetoacetate residue was converted to $\mathbf{S 1 4}$ without further purification. A solution of the unpurified acetoacetate was dissolved in $20 \mathrm{~mL}$ of $\mathrm{MeCN}$ and treated with $\mathrm{Et}_{3} \mathrm{~N}$. A solution of methanesulfonyl azide ${ }^{14}$ $(0.493 \mathrm{~g}, 4.07 \mathrm{mmol})$ in $10 \mathrm{~mL}$ of $\mathrm{MeCN}$ was added to the reaction mixture and the resultant yellow solution was stirred for $24 \mathrm{~h} .{ }^{15}$ A solution of $\mathrm{LiOH} \cdot \mathrm{H}_{2} \mathrm{O}(0.400 \mathrm{~g})$ in $10 \mathrm{~mL}$ of $\mathrm{H}_{2} \mathrm{O}$ was added and the solution was stirred for $24 \mathrm{~h}$. The reaction mixture was partitioned between $20 \mathrm{~mL}$ each of $\mathrm{CH}_{2} \mathrm{Cl}_{2}$ and $\mathrm{H}_{2} \mathrm{O}$. The aqueous layer was extracted with $2 \square 10 \mathrm{~mL}$ of $\mathrm{CH}_{2} \mathrm{Cl}_{2}$. The combined organic layers were dried $\left(\mathrm{Na}_{2} \mathrm{SO}_{4}\right)$ and concentrated in vacuo to provide a yellow oil. Purification by flash chromatography (8:92 - 90:10 EtOAc/hexanes) provided $\mathbf{S 1 4}$ as a yellow oil $(0.559 \mathrm{~g}, 67 \%):{ }^{1} \mathrm{H}$ NMR $\left(500 \mathrm{MHz}, \mathrm{CDCl}_{3}\right) \square 7.29(\mathrm{~m}, 15 \mathrm{H})$, 4.71 (br s, $1 \mathrm{H}), 4.52(\mathrm{~s}, 6 \mathrm{H}), 3.87$ (s, 6H); ${ }^{13} \mathrm{C} \mathrm{NMR}\left(125 \mathrm{MHz}, \mathrm{CDCl}_{3}\right) \square 165.9,138.2,128.3,127.51,127.48$, 84.6, 73.4, 68.0, 47.0; IR (thin film) 2864, 2111, 1693, 1373, $1099 \mathrm{~cm}^{-1}$. Anal. Calcd for $\mathrm{C}_{27} \mathrm{H}_{28} \mathrm{~N}_{2} \mathrm{O}_{5}$ : C, 70.42; H, 6.13; N, 6.08. Found: C, 70.19; H, 5.92; N, 5.96.

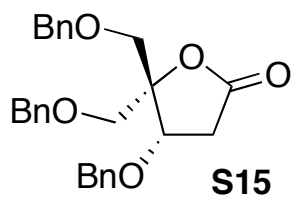

3-Benzyloxy-4,4-bis(benzyloxymethyl)dihydrofuran-1-one (S15). A $100 \mathrm{~mL}$, three-necked flask was fitted with two septa and a short Vigreux column filled with $3 \AA$ molecular sieves and topped with a reflux condenser before flame-drying the entire assembly under a positive flow of nitrogen. The dirhodium catalyst ${ }^{16}\left(\mathrm{Rh}_{2}(5 R\right.$ MEPY), $0.010 \mathrm{~g}, 0.013 \mathrm{mmol}$ ) was added to the three-necked flask, followed by $50 \mathrm{~mL}$ of $\mathrm{CH}_{2} \mathrm{Cl}_{2}$. The reaction mixture was heated to reflux under positive $\mathrm{N}_{2}$ pressure, and a solution of $\mathbf{S 1 4}(0.344 \mathrm{~g}, 0.747 \mathrm{mmol})$ in $10 \mathrm{~mL}$ of $\mathrm{CH}_{2} \mathrm{Cl}_{2}$ was added over $10 \mathrm{~h}$ with the aid of a syringe pump. The mixture was cooled to $22{ }^{\circ} \mathrm{C}$, concentrated in vacuo, and purified by flash chromatography (6:94 - 8:92 - 10:90 EtOAc/hexanes) to yield the product as a colorless oil $(0.225 \mathrm{~g}, 70 \%)$ : [ []$_{25}^{\mathrm{D}}{ }_{25} 50.0,[\mathrm{\square}]^{405}{ }_{25} 98.3\left(c 0.100, \mathrm{CHCl}_{3}\right) ;{ }^{1} \mathrm{H}$ NMR $(500 \mathrm{MHz}$, $\left.\mathrm{CDCl}_{3}\right) \square 7.31(\mathrm{~m}, 15 \mathrm{H}), 4.53(\mathrm{~m}, 6 \mathrm{H}), 4.30(\mathrm{dd}, J=7.0,3.5,1 \mathrm{H}), 3.82(\mathrm{~d}, J=10.6,1 \mathrm{H}), 3.80(\mathrm{~d}, J=10.6,1 \mathrm{H})$, $3.72(\mathrm{~d}, J=10.6,1 \mathrm{H}), 3.67(\mathrm{~d}, J=10.6,1 \mathrm{H}), 2.89(\mathrm{dd}, J=17.7,7.1,1 \mathrm{H}), 2.64(\mathrm{dd}, J=17.7,3.5,1 \mathrm{H}) ;{ }^{13} \mathrm{C}$ NMR $\left(125 \mathrm{MHz}, \mathrm{CDCl}_{3}\right) \square 174.9,137.8,137.5,137.3,128.5,128.3,127.9,127.8,127.64,127.59,127.54,127.51$, 88.9, 76.8, 73.8, 73.7, 72.0, 71.8, 69.3, 36.5; IR (thin film) 2925, 2867, 1782, $1097 \mathrm{~cm}^{-1}$; HRMS (CI/NH $\mathrm{NH}_{3} \mathrm{~m} / \mathrm{z}$ calcd for $\mathrm{C}_{27} \mathrm{H}_{29} \mathrm{O}_{5}(\mathrm{M}+\mathrm{H})^{+}$433.2015, found 433.2015. Anal. Calcd for $\mathrm{C}_{27} \mathrm{H}_{28} \mathrm{O}_{5}$ : C, 74.98; $\mathrm{H}, 6.53$. Found: C, 74.80; H, 6.47. The product was found to be $64 \%$ ee by chiral HPLC analysis (Chiracel $\square$ OD-H, $1 \mathrm{~mL} / \mathrm{min}$, 80:20 hexanes $/ i-\mathrm{PrOH}$ ), $t_{\mathrm{R}}=14.4 \mathrm{~min}$ (major) and $17.9 \mathrm{~min}$ (minor). The peaks were assigned by comparison to a chromatogram of racemic product formed with an achiral catalyst $\mathrm{Rh}_{2}(\text { caprolactam })_{4}$. The absolute configuration of the major product was not determined. 


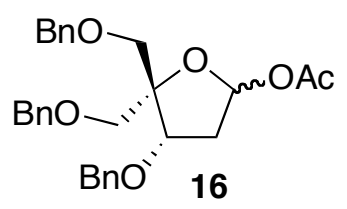

1-Acetoxy-3-benzyloxy-4,4-bis-benzyloxymethyltetrahydrofuran (16). The standard reductive acylation procedure was followed with lactone $\mathbf{S 1 5}(0.116 \mathrm{~g}, 0.268 \mathrm{mmol})$ in $5 \mathrm{~mL}$ of $\mathrm{CH}_{2} \mathrm{Cl}_{2}$, with DIBAL-H $(0.36 \mathrm{~mL}$, $0.54 \mathrm{mmol})$, pyridine $(0.130 \mathrm{~mL}, 1.61 \mathrm{mmol})$, DMAP $(0.144 \mathrm{~g}, 1.17 \mathrm{mmol})$, and $\mathrm{Ac}_{2} \mathrm{O}(0.530 \mathrm{~mL}, 4.29 \mathrm{mmol})$. ${ }^{1} \mathrm{H}$ NMR spectroscopic analysis of the unpurified product showed a pair of diastereomers in a ratio of 63:37. Purification by flash chromatography (70:30 - 100:0 $\mathrm{CH}_{2} \mathrm{Cl}_{2}$ /hexanes) yielded acetate $\mathbf{1 6}$ as a colorless oil $(0.087 \mathrm{~g}, 68 \%)$. The purified product was characterized as a mixture of diastereomers: ${ }^{1} \mathrm{H}$ NMR $(500 \mathrm{MHz}$, $\left.\mathrm{CDCl}_{3}\right) \square 7.28(\mathrm{~m}, 15 \mathrm{H}), 6.34(\mathrm{~m}, 1 \mathrm{H}), 4.54(\mathrm{~m}, 6 \mathrm{H}), 4.31(\mathrm{t}, J=7.4,0.6 \mathrm{H}), 4.20(\mathrm{~m}, 0.4 \mathrm{H}), 3.87(\mathrm{~d}, J=10.3$, $0.4 \mathrm{H}), 3.77(\mathrm{~d}, J=10.3,0.4 \mathrm{H}), 3.72(\mathrm{~m}, 3.2 \mathrm{H}), 2.40(\mathrm{~m}, 1 \mathrm{H}), 2.25(\mathrm{~m}, 1 \mathrm{H}), 2.0(\mathrm{~s}, 1.2 \mathrm{H}), 1.93(\mathrm{~s}, 1.8 \mathrm{H}) ;{ }^{13} \mathrm{C}$ NMR (125 MHz, $\left.\mathrm{CDCl}_{3}\right) \square$ 170.5, 170.0, 138.5, 138.3, 138.21, 138.17, 138.1, 128.3, 128.24, 128.18, 127.7, 127.6, 127.54, 127.51, 127.48, 127.44, 127.38, 127.3, 127.2, 98.8, 97.2, 89.8, 79.8, 79.6, 73.6, 73.4, 72.3, 72.0, 71.7, 70.3, 69.7, 38.8, 38.2, 21.4, 21.3; IR (thin film) 2922, 2863, 1743, 1239, $1101 \mathrm{~cm}^{-1}$; HRMS (EI) $\mathrm{m} / z$ calcd for $\mathrm{C}_{29} \mathrm{H}_{33} \mathrm{O}_{6}(\mathrm{M}+\mathrm{H})^{+}$477.2277, found 477.2267. Anal. Calcd for $\mathrm{C}_{29} \mathrm{H}_{32} \mathrm{O}_{6}: \mathrm{C}, 73.09 ; \mathrm{H}, 6.77$. Found: C, 73.37; H, 6.83 .

\section{E. (4S)-1-Acetoxy-4-t-butyldiphenylsiloxymethyl)tetrahydrofuran (18b) Synthetic Scheme.}
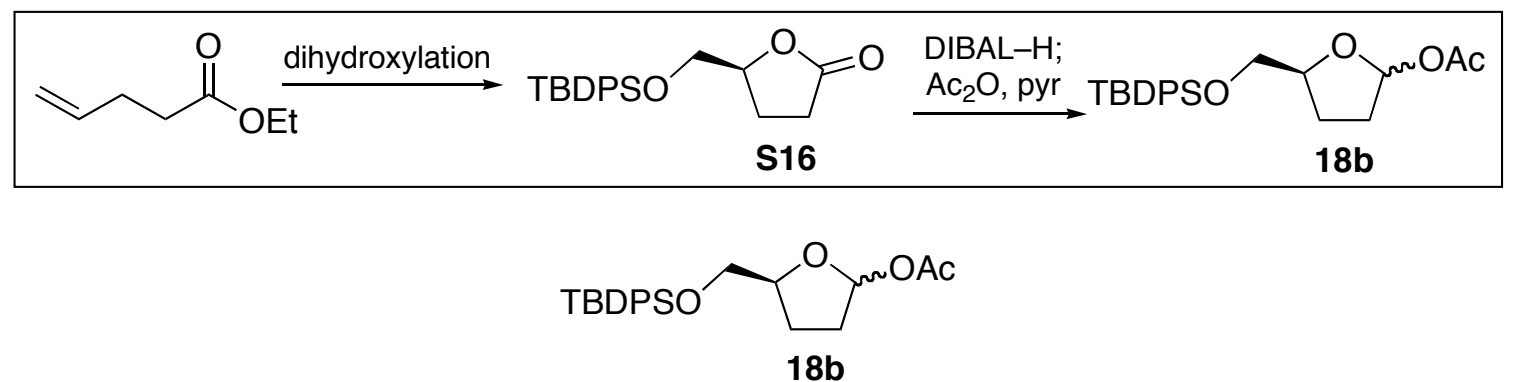

(4S)-1-Acetoxy-4-t-butyldiphenylsiloxymethyl)tetrahydrofuran (18b). The standard reductive acylation procedure was followed with lactone $\mathbf{S 1 6}{ }^{17}(0.35 \mathrm{~g}, 0.98 \mathrm{mmol})$ in $5 \mathrm{~mL}$ of $\mathrm{CH}_{2} \mathrm{Cl}_{2}$, with DIBAL-H $(0.8 \mathrm{~mL}$, $1.2 \mathrm{mmol})$, pyridine $(0.317 \mathrm{~mL}, 3.92 \mathrm{mmol})$, DMAP $(0.144 \mathrm{~g}, 1.18 \mathrm{mmol})$, and $\mathrm{Ac}_{2} \mathrm{O}(0.46 \mathrm{~mL}, 4.9 \mathrm{mmol})$. After $24 \mathrm{~h}$ at $22{ }^{\circ} \mathrm{C}$, additional pyridine $(0.317 \mathrm{~mL}, 3.92 \mathrm{mmol})$ and $\mathrm{Ac}_{2} \mathrm{O}(0.46 \mathrm{~mL}, 4.9 \mathrm{mmol})$ were added and the solution was stirred for $24 \mathrm{~h} .{ }^{1} \mathrm{H}$ NMR spectroscopic analysis of the unpurified product showed a pair of diastereomers in a ratio of 80:20. Purification by flash chromatography (10:90 EtOAc/hexanes) yielded acetate $\mathbf{1 8 b}$ as a colorless oil $(0.341 \mathrm{~g}, 87 \%)$. The purified product was characterized as a mixture of diastereomers: ${ }^{1} \mathrm{H}$ NMR (500 MHz, $\left.\mathrm{CDCl}_{3}\right) \square 7.70-7.65(\mathrm{~m}, 4 \mathrm{H}), 7.43-7.36(\mathrm{~m}, 6 \mathrm{H}), 6.34(\mathrm{~d}, J=4.5,0.2 \mathrm{H}), 6.28(\mathrm{~d}, J=2.7$, $0.8 \mathrm{H}), 4.37(\mathrm{~m}, 0.2 \mathrm{H}), 4.24(\mathrm{~m}, 0.8 \mathrm{H}), 3.58(\mathrm{~m}, 2 \mathrm{H}), 2.04(\mathrm{~m}, 4.6 \mathrm{H}), 1.90(\mathrm{~s}, 2.4 \mathrm{H}), 1.07(\mathrm{~s}, 7.2 \mathrm{H}), 1.05(\mathrm{~s}$, $1.8 \mathrm{H}) ;{ }^{13} \mathrm{C}$ NMR $\left(125 \mathrm{MHz}, \mathrm{CDCl}_{3}\right) \square 170.4,135.54,135.51,133.5,133.4,129.60,129.58,127.6,99.5,98.8$, 82.1, 80.5, 66.4, 65.6, 32.5, 31.8, 26.7, 25.1, 24.6, 21.3, 19.2; HRMS (CI/isobutane) $m / z$ calcd for $\mathrm{C}_{21} \mathrm{H}_{27} \mathrm{O}_{2} \mathrm{Si}$ $\left(\mathrm{M}-\mathrm{C}_{2} \mathrm{H}_{3} \mathrm{O}_{2}\right)^{+} 339.1780$, found 339.1771 .

\section{F. $\left(4 R^{*}\right)-1$-Acetoxy-4-(3-phenylpropyl)tetrahydrofuran (18c)} Synthetic Scheme. 

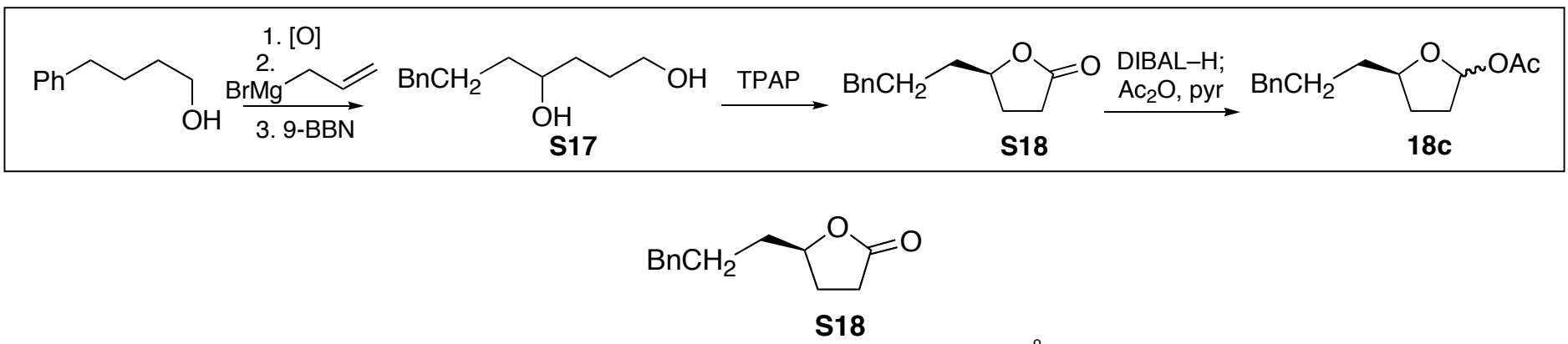

4-(3-Phenylpropyl)dihydrofuran-1-one (S18). Pulverized $4 \AA$ molecular sieves $(0.3 \mathrm{~g})$ and $N$ methylmorpholine- $N$-oxide $(0.18 \mathrm{~g}, 1.5 \mathrm{mmol})$ were weighed into a flask. A solution of diol S17 $\mathbf{S}^{18}(0.146 \mathrm{~g}$, $0.701 \mathrm{mmol})$ in $\mathrm{MeCN}(7 \mathrm{~mL})$ was added followed by tetrapropylammonium perruthenate $(0.024 \mathrm{~g}, 0.071$ mmol). ${ }^{19}$ The mixture was stirred for $14 \mathrm{~h}$. Silica gel $(3-4 \mathrm{~g})$ was added and the mixture was concentrated in vacuo and purified by flash chromatography (30:70 EtOAc/hexanes) to afford the product as a colorless oil $(0.126 \mathrm{~g}, 88 \%):{ }^{1} \mathrm{H}$ NMR $\left(500 \mathrm{MHz}, \mathrm{CDCl}_{3}\right) \square 7.28(\mathrm{t}, J=7.5,2 \mathrm{H}), 7.21-7.16(\mathrm{~m}, 3 \mathrm{H}), 4.49(\mathrm{~m}, 1 \mathrm{H}), 2.66(\mathrm{t}, J$ $=7.4,2 \mathrm{H}), 2.51(\mathrm{dd}, J=9.6,6.9,2 \mathrm{H}), 2.30($ sextet, $J=6.6,1 \mathrm{H}), 1.83(\mathrm{~m}, 2 \mathrm{H}), 1.72(\mathrm{~m}, 2 \mathrm{H}), 1.63(\mathrm{~m}, 1 \mathrm{H}) ;{ }^{13} \mathrm{C}$ NMR (125 MHz, $\left.\mathrm{CDCl}_{3}\right) \square 177.1,141.5,128.3,125.8,80.7,35.3,34.9,28.7,27.8,26.9$; IR (thin film) 3026, 2862, 1775, $1179 \mathrm{~cm}^{-1}$; HRMS (EI) $m / z$ calcd for $\mathrm{C}_{13} \mathrm{H}_{16} \mathrm{O}_{2}(\mathrm{M})^{+}$204.1150, found 204.1153. Anal. Calcd for $\mathrm{C}_{13} \mathrm{H}_{16} \mathrm{O}_{2}$ : C, 76.44; H, 7.90. Found: C, 76.20; H, 8.02.

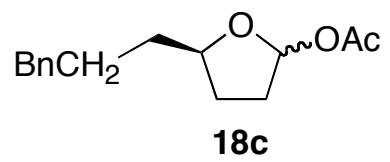

(4R*)-1-Acetoxy-4-(3-phenylpropyl)tetrahydrofuran (18c). The standard reductive acylation procedure was followed with lactone $\mathbf{S 1 8}(0.065 \mathrm{~g}, 0.32 \mathrm{mmol})$ in $5 \mathrm{~mL}$ of $\mathrm{CH}_{2} \mathrm{Cl}_{2}$, with DIBAL-H $(0.26 \mathrm{~mL}, 0.39 \mathrm{mmol})$, pyridine $(0.105 \mathrm{~mL}, 1.30 \mathrm{mmol})$, DMAP $(0.047 \mathrm{~g}, 0.38 \mathrm{mmol})$, and $\mathrm{Ac}_{2} \mathrm{O}(0.151 \mathrm{~mL}, 1.60 \mathrm{mmol})$. After $24 \mathrm{~h}$ at $22{ }^{\circ} \mathrm{C}$, additional pyridine $(0.105 \mathrm{~mL}, 1.30 \mathrm{mmol})$ and $\mathrm{Ac}_{2} \mathrm{O}(0.151 \mathrm{~mL}, 1.60 \mathrm{mmol})$ were added and the solution was stirred for $24 \mathrm{~h}$. ${ }^{1} \mathrm{H}$ NMR spectroscopic analysis of the unpurified product showed a pair of diastereomers in a ratio of 50:50. Purification by bulb-to-bulb distillation $\left(0.05\right.$ Torr, $\left.130{ }^{\circ} \mathrm{C}\right)$ afforded acetate $18 \mathrm{c}$ as a colorless oil $(0.067 \mathrm{~g}, 85 \%)$. The purified product was characterized as a mixture of diastereomers: ${ }^{1} \mathrm{H}$ $\operatorname{NMR}\left(500 \mathrm{MHz}, \mathrm{CDCl}_{3}\right) \square 7.29-7.26(\mathrm{~m}, 2 \mathrm{H}), 7.19-7.17(\mathrm{~m}, 3 \mathrm{H}), 6.28(\mathrm{dd}, J=5.0,1.4,0.5 \mathrm{H}), 6.22(\mathrm{t}, J=2.4$, $0.5 \mathrm{H}), 4.21$ (quintet, $J=6.4,0.5 \mathrm{H}), 4.10(\mathrm{~m}, 0.5 \mathrm{H}), 2.64(\mathrm{~m}, 2 \mathrm{H}), 2.13(\mathrm{~m}, 1 \mathrm{H}), 2.03(\mathrm{~m}, 2.5 \mathrm{H}), 1.99(\mathrm{~s}, 1.5 \mathrm{H})$, $1.97(\mathrm{~m}, 1 \mathrm{H}), 1.78-1.46(\mathrm{~m}, 5 \mathrm{H}) ;{ }^{13} \mathrm{C} \mathrm{NMR}\left(125 \mathrm{MHz}, \mathrm{CDCl}_{3}\right) \square 170.5,170.4,142.2,142.1,128.3,128.2$, 125.7, 125.6, 99.0, 98.7, 81.9, 80.0, 36.2, 35.7, 35.6, 34.8, 32.8, 31.7, 28.5, 28.4, 27.6, 28.5, 21.4, 21.3; IR (thin film) 3085, 2862, 1742, $1006 \mathrm{~cm}^{-1}$; HRMS (CI/isobutane) $m / z$ calcd for $\mathrm{C}_{13} \mathrm{H}_{16} \mathrm{O}\left(\mathrm{M}-\mathrm{C}_{2} \mathrm{H}_{4} \mathrm{O}_{2}\right)^{+} 188.1201$, found 188.1197. Anal. Calcd for $\mathrm{C}_{15} \mathrm{H}_{20} \mathrm{O}_{3}$ : C, 72.55; H, 8.12. Found: C, 72.26; H, 8.05.

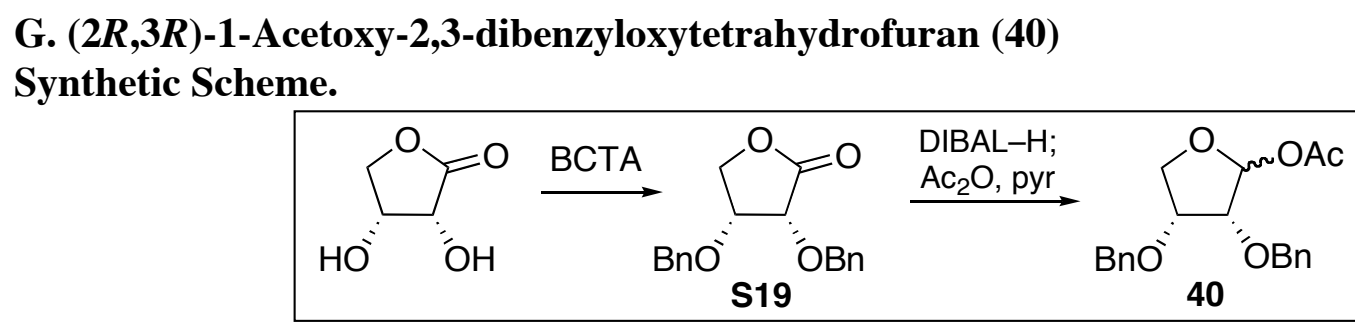




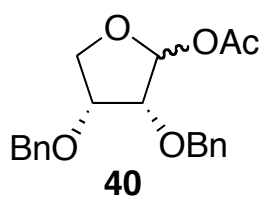

$(2 R, 3 R)$-1-Acetoxy-2,3-dibenzyloxytetrahydrofuran (40). The standard reductive acylation procedure was followed with lactone $\mathbf{S 1 9}^{20}(0.160 \mathrm{~g}, 0.536 \mathrm{mmol})$ in $5 \mathrm{~mL}$ of $\mathrm{CH}_{2} \mathrm{Cl}_{2}$ with DIBAL-H $(0.43 \mathrm{~mL}, 0.65 \mathrm{mmol})$, pyridine $(0.170 \mathrm{~mL}, 2.16 \mathrm{mmol})$, DMAP $(0.080 \mathrm{~g}, 0.65 \mathrm{mmol})$, and $\mathrm{Ac}_{2} \mathrm{O}(0.200 \mathrm{~mL}, 2.16 \mathrm{mmol})$. GC and ${ }^{1} \mathrm{H}$ NMR spectroscopic analysis of the unpurified product showed a pair of diastereomers in a ratio of 85:15. Purification of the resultant residue by flash chromatography (0:100 - 5:95 EtOAc/hexanes) yielded 40 as a colorless oil $(0.185 \mathrm{~g}, 81 \%)$. The two diastereomers were separable in small amounts with careful chromatography. Elemental analysis, HRMS, and IR data were obtained for $\mathbf{4 0}$ as a mixture of diastereomers: IR (thin film) 3031, 2934, 2898, 1745, 1454, $1231 \mathrm{~cm}^{-1}$; HRMS (EI) $m / z$ calcd for $\mathrm{C}_{20} \mathrm{H}_{22} \mathrm{O}_{5} \mathrm{Na}(\mathrm{M}+\mathrm{Na})^{+}$ 365.1365, found 365.1352. Anal. Calcd for $\mathrm{C}_{20} \mathrm{H}_{22} \mathrm{O}_{5}: \mathrm{C}, 70.16 ; \mathrm{H}, 6.48$. Found: C, 70.34; H, 6.42.

Major isomer of 40: ${ }^{1} \mathrm{H} \mathrm{NMR}\left(500 \mathrm{MHz}, \mathrm{CDCl}_{3}\right) \square 7.40-7.30(\mathrm{~m}, 10 \mathrm{H}), 6.27(\mathrm{~d}, J=4.5,1 \mathrm{H}), 4.76(\mathrm{~d}, J=$ $12.3,1 \mathrm{H}), 4.68(\mathrm{~d}, J=12.2,1 \mathrm{H}), 4.64(\mathrm{~d}, J=12.2,1 \mathrm{H}), 4.63(\mathrm{~d}, J=12.3,1 \mathrm{H}), 4.08(\mathrm{dd}, J=9.8,2.5,1 \mathrm{H}), 4.05$ $(\mathrm{ddd}, J=5.6,5.2,2.5,1 \mathrm{H}), 3.99(\mathrm{dd}, J=9.7,5.1,1 \mathrm{H}), 3.92(\mathrm{dd}, J=5.8,4.6,1 \mathrm{H}), 2.16(\mathrm{~s}, 3 \mathrm{H}) ;{ }^{13} \mathrm{C}$ NMR $(125$ $\left.\mathrm{MHz}, \mathrm{CDCl}_{3}\right) \square 171.2,138.5,137.9,129.0,128.8,128.5,128.30,128.28,128.2,94.3,78.7,74.2,73.5,72.8$, 22.0 .

Minor isomer of 40: ${ }^{1} \mathrm{H} \mathrm{NMR}\left(500 \mathrm{MHz}, \mathrm{CDCl}_{3}\right) \square 7.38-7.28(\mathrm{~m}, 10 \mathrm{H}), 6.22(\mathrm{~d}, J=1.4,1 \mathrm{H}), 4.73(\mathrm{~d}, J=$ $12.1,1 \mathrm{H}), 4.66(\mathrm{~d}, J=12.1,1 \mathrm{H}), 4.58(\mathrm{~d}, J=11.9,1 \mathrm{H}), 4.50(\mathrm{~d}, J=11.9,1 \mathrm{H}), 4.22(\mathrm{td}, J=6.2,4.8,1 \mathrm{H}), 4.13$ $(\mathrm{dd}, J=8.9,6.4,1 \mathrm{H}), 4.02(\mathrm{dd}, J=8.9,6.2,1 \mathrm{H}), 3.96(\mathrm{dd}, J=4.7,1.4,1 \mathrm{H}), 2.03(\mathrm{~s}, 3 \mathrm{H}) ;{ }^{13} \mathrm{C} \mathrm{NMR}(125 \mathrm{MHz}$, $\left.\mathrm{CDCl}_{3}\right) \square 170.3,138.1,137.9,128.91,128.90,128.5,128.41,128.35,128.2,100.4,80.3,76.8,72.77,72.76$, 71.1, 21.6.

\section{H. (2S,3R)-1-Acetoxy-2,3-dibenzyloxytetrahydrofuran (42) Synthetic Scheme.}
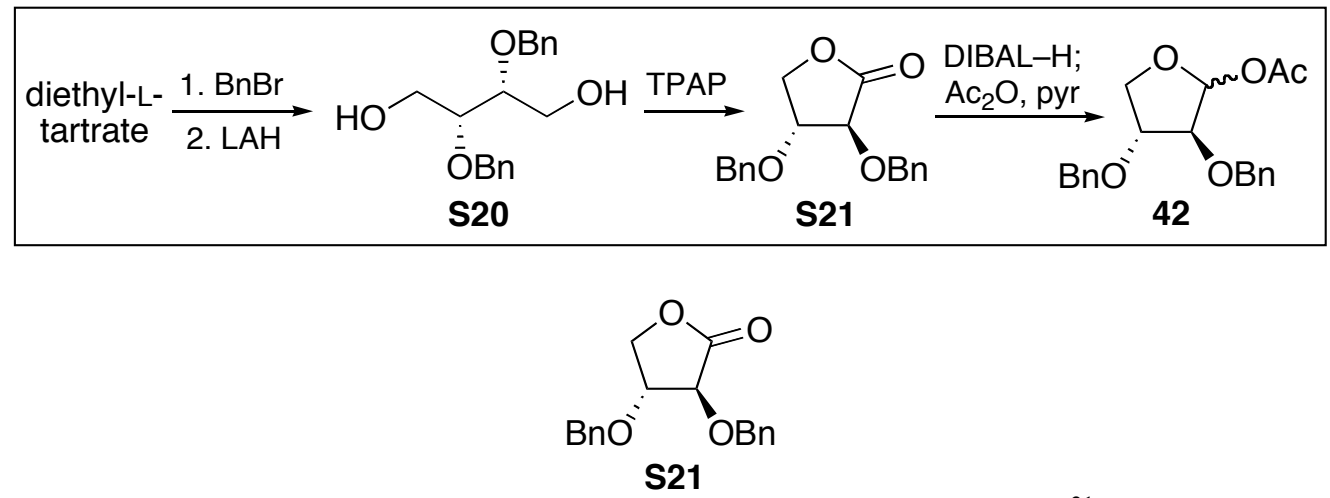

(2S,3R)-2,3-Dibenzyloxydihydrofuran-1-one (S21). To a solution of diol $\mathbf{S 2 0}^{21}(4.11 \mathrm{~g}, 13.6 \mathrm{mmol})$ in $\mathrm{MeCN}$ $(140 \mathrm{~mL})$ was added 4-methylmorpholine- $N$-oxide $(4.78 \mathrm{~g}, 40.8 \mathrm{mmol})$. This mixture was transferred by cannula into a flask containing crushed, flame-dried $4 \AA$ molecular sieves. Tetrapropylammonium perruthenate $(0.717 \mathrm{~g}, 2.04 \mathrm{mmol})$ was added to the reaction mixture. After $20 \mathrm{~h}$ at $22{ }^{\circ} \mathrm{C}$, silica gel $(3.5 \mathrm{~g})$ was added and the mixture was concentrated in vacuo. Purification by flash chromatography (20:80 EtOAc/hexanes) provided the product as a colorless oil (3.98 g, 98\%): ${ }^{1} \mathrm{H}$ NMR $\left(500 \mathrm{MHz}, \mathrm{CDCl}_{3}\right) \square 7.40-7.24(\mathrm{~m}, 10 \mathrm{H}), 5.01(\mathrm{~d}, J=$ $11.6,1 \mathrm{H}), 4.73(\mathrm{~d}, J=11.6,1 \mathrm{H}), 4.61(\mathrm{~d}, J=11.7,1 \mathrm{H}), 4.51(\mathrm{~d}, J=11.7,1 \mathrm{H}), 4.38(\mathrm{dd}, J=9.3,6.7,1 \mathrm{H}), 4.30$ $(\mathrm{q}, J=6.1,1 \mathrm{H}), 4.21(\mathrm{~d}, J=5.9,1 \mathrm{H}), 4.04(\mathrm{dd}, J=9.4,6.2,1 \mathrm{H}) ;{ }^{13} \mathrm{C} \mathrm{NMR}\left(125 \mathrm{MHz}, \mathrm{CDCl}_{3}\right) \square 173.6,137.4$, 137.1, 129.1, 129.0, 128.9, 128.72, 128.70, 128.3, 78.9, 77.9, 72.9, 72.7, 69.5; IR (thin film) 3032, 2872, 1794, 1455, $747 \mathrm{~cm}^{-1}$. Anal. Calcd for $\mathrm{C}_{18} \mathrm{H}_{18} \mathrm{O}_{4}: \mathrm{C}, 72.47 ; \mathrm{H}, 6.08$. Found: C, 72.60; H, 6.32. 


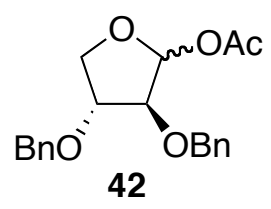

(2S,3R)-1-Acetoxy-2,3-dibenzyloxytetrahydrofuran (42). The standard reductive acylation procedure was followed with lactone $\mathbf{S 2 1}{ }^{22}(2.20 \mathrm{~g}, 7.37 \mathrm{mmol})$ in $75 \mathrm{~mL}$ of $\mathrm{CH}_{2} \mathrm{Cl}_{2}$, with DIBAL-H (5.9 mL, $\left.8.9 \mathrm{mmol}\right)$, pyridine $(2.38 \mathrm{~mL}, 29.5 \mathrm{mmol})$, DMAP $(1.09 \mathrm{~g}, 8.85 \mathrm{mmol})$, and $\mathrm{Ac}_{2} \mathrm{O}(3.48 \mathrm{~mL}, 36.9 \mathrm{mmol})$. ${ }^{1} \mathrm{H}$ NMR spectroscopic analysis of the unpurified product showed a pair of diastereomers in a ratio of 56:44. Purification by flash chromatography (2:98 EtOAc/hexanes) yielded acetate $\mathbf{4 2}$ as a colorless oil $(2.41 \mathrm{~g}, 96 \%)$. The purified product was characterized as a mixture of diastereomers: ${ }^{1} \mathrm{H}$ NMR $\left(500 \mathrm{MHz}, \mathrm{CDCl}_{3}\right) \square 7.43-7.33$ (m, $18 \mathrm{H}), 6.36(\mathrm{~d}, J=4.3,1 \mathrm{H}), 6.24(\mathrm{~s}, 0.8 \mathrm{H}), 4.73(\mathrm{~d}, J=11.9,0.8 \mathrm{H}), 4.67(\mathrm{~d}, J=11.6,2 \mathrm{H}), 4.60(\mathrm{~m}, 4.4 \mathrm{H}), 4.34$ $(\mathrm{m}, 1.8 \mathrm{H}), 4.26(\mathrm{dd}, J=9.5,7.0,1 \mathrm{H}), 4.22(\mathrm{dd}, J=5.4,4.3,1 \mathrm{H}), 4.20(\mathrm{~m}, 0.8 \mathrm{H}), 4.15(\mathrm{~m}, 0.8 \mathrm{H}), 4.07(\mathrm{dd}, J=$ 9.4, 5.0, 0.8H), $3.90(\mathrm{dd}, J=9.5,4.6,1 \mathrm{H}), 2.14(\mathrm{~s}, 5.4 \mathrm{H}) ;{ }^{13} \mathrm{C} \mathrm{NMR}\left(125 \mathrm{MHz}, \mathrm{CDCl}_{3}\right) \square$ 170.4, 170.3, 137.9, $137.7,137.5,137.4,128.71,128.68,128.3,128.24,128.20,128.15,128.12,128.10,128.0,127.9,100.9,95.4$, 86.4, 84.0, 82.3, 80.8, 73.4, 72.9, 72.4, 72.30, 72.25, 71.0, 21.5, 21.4; IR (thin film) 2898, 1737, 1586, 1454, $1239 \mathrm{~cm}^{-1}$; HRMS (EI) $\mathrm{m} / \mathrm{z}$ calcd for $\mathrm{C}_{20} \mathrm{H}_{22} \mathrm{O}_{5} \mathrm{Na}(\mathrm{M}+\mathrm{Na})^{+} 365.1365$, found 365.1367 .

I. $\left(2 S^{*}, 3 S^{*}\right)$-1-Acetoxy-3-benzyloxy-2-methyltetrahydrofuran (50). Synthetic Scheme.
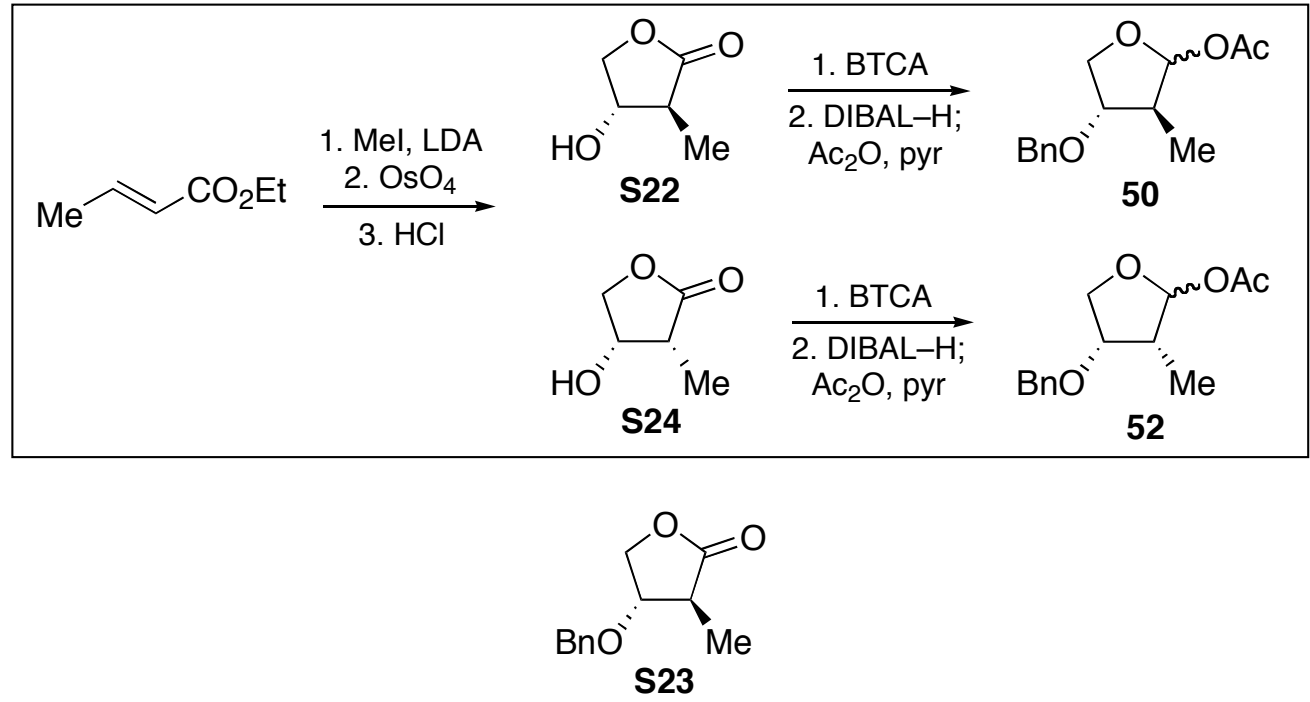

$\left(2 S^{*}, 3 S^{*}\right)$-3-Benzyloxy-2-methyldihydrofuran-1-one $(\mathbf{S 2 3})$. To a cooled $\left(0{ }^{\circ} \mathrm{C}\right)$ solution of lactone $\mathbf{S 2 2}^{23}$ $(1.31 \mathrm{~g}, 11.3 \mathrm{mmol})$ in $\mathrm{CH}_{2} \mathrm{Cl}_{2}(56 \mathrm{~mL})$ and cyclohexane $(56 \mathrm{~mL})$ was added benzyl trichloroacetimidate (4.19 $\mathrm{mL}, 22.6 \mathrm{mmol})$ followed by dropwise addition of $\mathrm{BF}_{3} \cdot \mathrm{OEt}_{2}(0.29 \mathrm{~mL}, 2.3 \mathrm{mmol}){ }^{24}$ The mixture was stirred for $12 \mathrm{~h}$ as a white precipitate formed (trichloroacetamide). Hexanes was added $(100 \mathrm{~mL})$, the mixture was filtered, and the filtrate was washed with saturated aqueous $\mathrm{NaHCO}_{3}(50 \mathrm{~mL})$, dried $\left(\mathrm{Na}_{2} \mathrm{SO}_{4}\right)$, and concentrated in vacuo. The resulting oil was purified by recrystallization (hexane $\left./ \mathrm{CH}_{2} \mathrm{Cl}_{2}\right)$ and flash chromatography $(10: 90-$ 30:70 EtOAc/hexanes, then 5:95 - 20:80 EtOAc/PhH) to afford $\mathbf{S 2 3}$ as a colorless oil $(1.23 \mathrm{~g}, 53 \%):{ }^{1} \mathrm{H} \mathrm{NMR}$ $\left(500 \mathrm{MHz}, \mathrm{CDCl}_{3}\right) \square 7.38-7.33(\mathrm{~m}, 2 \mathrm{H}), 7.32-7.30(\mathrm{~m}, 3 \mathrm{H}), 4.59(\mathrm{~d}, J=11.8,1 \mathrm{H}), 4.54(\mathrm{~d}, J=11.8,1 \mathrm{H}), 4.36$ $(\mathrm{dd}, J=9.7,6.0,1 \mathrm{H}), 4.13(\mathrm{dd}, J=9.7,4.9,1 \mathrm{H}), 3.99(\mathrm{~m}, 1 \mathrm{H}), 2.68(\mathrm{dq}, J=7.5,5.4,1 \mathrm{H}), 1.30(\mathrm{~d}, J=7.5,3 \mathrm{H})$; ${ }^{13} \mathrm{C}$ NMR $\left(125 \mathrm{MHz}, \mathrm{CDCl}_{3}\right) \square 177.8,137.1,128.6,128.1,127.7,80.5,71.8,70.6,40.9$, 13.6; IR (thin film) 
2976, 2879, $1775 \mathrm{~cm}^{-1}$; HRMS (FAB) $m / z$ calcd for $\mathrm{C}_{12} \mathrm{H}_{15} \mathrm{O}_{3}(\mathrm{M}+\mathrm{H})^{+}$207.1021, found 207.1018. Anal. Calcd for $\mathrm{C}_{12} \mathrm{H}_{14} \mathrm{O}_{3}$ : C, 69.89; H, 6.84. Found: C, 69.92; H, 6.81.

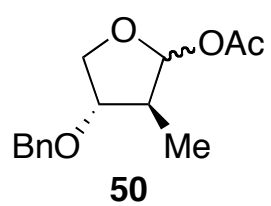

$\left(2 S^{*}, 3 S^{*}\right)$-1-Acetoxy-3-benzyloxy-2-methyltetrahydrofuran $(50)$. The standard reductive acylation procedure was followed with lactone $\mathbf{S 2 3}(0.447 \mathrm{~g}, 2.17 \mathrm{mmol})$ in $22 \mathrm{~mL}$ of $\mathrm{CH}_{2} \mathrm{Cl}_{2}$, with DIBAL-H $(1.7 \mathrm{~mL}$, $2.6 \mathrm{mmol})$, pyridine $(0.702 \mathrm{~mL}, 8.68 \mathrm{mmol})$, DMAP $(0.319 \mathrm{~g}, 2.60 \mathrm{mmol})$, and $\mathrm{Ac}_{2} \mathrm{O}(1.03 \mathrm{~mL}, 10.9 \mathrm{mmol})$. After $24 \mathrm{~h}$ at $22{ }^{\circ} \mathrm{C}$, additional pyridine $(0.702 \mathrm{~mL}, 8.68 \mathrm{mmol})$ and $\mathrm{Ac}_{2} \mathrm{O}(1.03 \mathrm{~mL}, 10.9 \mathrm{mmol})$ were added and the solution was stirred for $24 \mathrm{~h} .{ }^{1} \mathrm{H}$ NMR spectroscopic analysis of the unpurified product showed a pair of diastereomers in a ratio of 80:20. Purification by flash chromatography (5:95 - 25:75 EtOAc/hexanes) yielded acetate $\mathbf{5 0}$ as a colorless oil $(0.362 \mathrm{~g}, 67 \%)$.

Major isomer of 50: ${ }^{1} \mathrm{H}$ NMR (500 MHz, $\left.\mathrm{CDCl}_{3}\right) \square 7.35-7.27(\mathrm{~m}, 5 \mathrm{H}), 5.89(\mathrm{~s}, 1 \mathrm{H}), 4.55(\mathrm{~d}, J=11.9,1 \mathrm{H})$, 4.49 (d, $J=11.9,1 \mathrm{H}), 4.19(\mathrm{dd}, J=9.7,5.9,1 \mathrm{H}), 4.05(\mathrm{dd}, J=9.7,4.1,1 \mathrm{H}), 3.80(\mathrm{~m}, 1 \mathrm{H}), 2.49(\mathrm{~m}, 1 \mathrm{H}), 2.06$ (s, 3H), $1.09(\mathrm{~d}, J=7.5,3 \mathrm{H}) ;{ }^{13} \mathrm{C}$ NMR $\left(125 \mathrm{MHz}, \mathrm{CDCl}_{3}\right) \square 170.5,137.9,128.4,127.7,127.5,103.8,83.6$, 73.3, 71.5, 44.8, 21.3, 15.7; IR (thin film) 2971, 2881, 1738, $1008 \mathrm{~cm}^{-1}$; HRMS (CI/isobutane) $\mathrm{m} / z$ calcd for $\mathrm{C}_{14} \mathrm{H}_{18} \mathrm{O}_{4} \mathrm{Na}(\mathrm{M}+\mathrm{Na})^{+} 273.1103$, found 273.1099. Anal. Calcd for $\mathrm{C}_{14} \mathrm{H}_{18} \mathrm{O}_{4}: \mathrm{C}, 67.18 ; \mathrm{H}, 7.25$. Found: $\mathrm{C}$, $67.16 ; \mathrm{H}, 7.18$.

\section{J. $\left(2 R^{*}, 3 S^{*}\right)$-1-Acetoxy-3-benzyloxy-2-methyltetrahydrofuran (52)}

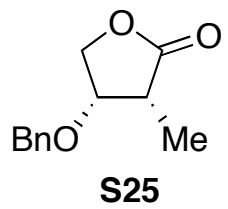

$\left(2 R^{*}, 3 S^{*}\right)$-3-Benzyloxy-2-methyldihydrofuran-1-one (S25). To a cooled $\left(0{ }^{\circ} \mathrm{C}\right)$ solution of lactone $\mathbf{S 2 4} \mathbf{4}^{25}$ $(2.10 \mathrm{~g}, 18.1 \mathrm{mmol})$ in $\mathrm{CH}_{2} \mathrm{Cl}_{2}(90 \mathrm{~mL})$ and cyclohexane $(90 \mathrm{~mL})$ was added benzyl trichloroacetimidate $(6.72$ $\mathrm{mL}, 36.2 \mathrm{mmol})$ followed by dropwise addition of $\mathrm{BF}_{3} \cdot \mathrm{OEt}_{2}(0.50 \mathrm{~mL}, 3.9 \mathrm{mmol}){ }^{24}$ The mixture was stirred for $12 \mathrm{~h}$ as a white precipitate formed (trichloroacetamide). Hexanes was added $(150 \mathrm{~mL}$ ), the mixture was filtered, and the filtrate was washed with saturated aqueous $\mathrm{NaHCO}_{3}(50 \mathrm{~mL})$, dried $\left(\mathrm{Na}_{2} \mathrm{SO}_{4}\right)$, and concentrated in vacuo. The resulting oil was purified by flash chromatography $(2 \square$ 10:90 - 40:60 EtOAc/hexane, then 5:95 20:80 EtOAc/ $\mathrm{PhH})$ to afford the product as a colorless oil $(1.40 \mathrm{~g}, 38 \%):{ }^{1} \mathrm{H}$ NMR $\left(500 \mathrm{MHz}, \mathrm{CDCl}_{3}\right) \square$ $7.38-7.34(\mathrm{~m}, 2 \mathrm{H}), 7.32-7.29(\mathrm{~m}, 3 \mathrm{H}), 4.65(\mathrm{~d}, J=12.1,1 \mathrm{H}), 4.51(\mathrm{~d}, J=12.1,1 \mathrm{H}), 4.38(\mathrm{~d}, J=10.1,1 \mathrm{H})$, $4.22(\mathrm{~m}, 1 \mathrm{H}), 4.19(\mathrm{dd}, J=10.1,3.6,1 \mathrm{H}), 2.65(\mathrm{qd}, J=7.3,5.5,1 \mathrm{H}), 1.31(\mathrm{~d}, J=7.3,3 \mathrm{H}) ;{ }^{13} \mathrm{C}$ NMR $(125$ $\left.\mathrm{MHz}, \mathrm{CDCl}_{3}\right) \mathrm{Q} 178.3,137.2,128.5,127.9,127.4,76.2,71.4,70.5,39.3,8.5$; IR (thin film) 3032, 2942, 1776 $\mathrm{cm}^{-1}$; HRMS (FAB) $m / z$ calcd for $\mathrm{C}_{12} \mathrm{H}_{15} \mathrm{O}_{3}(\mathrm{M}+\mathrm{H})^{+} 207.1021$, found 207.1017.

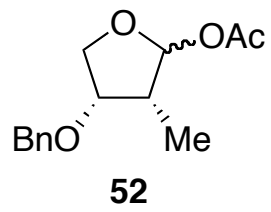

$\left(2 R^{*}, 3 S^{*}\right)$-1-Acetoxy-3-benzyloxy-2-methyltetrahydrofuran (52). The standard reductive acylation procedure was followed with lactone $\mathbf{S 2 5}(0.472 \mathrm{~g}, 2.29 \mathrm{mmol})$ in $23 \mathrm{~mL}$ of $\mathrm{CH}_{2} \mathrm{Cl}_{2}$, with DIBAL-H $(1.8 \mathrm{~mL}$, 
$2.7 \mathrm{mmol})$, pyridine $(0.337 \mathrm{~mL}, 9.16 \mathrm{mmol})$, DMAP $(0.337 \mathrm{~g}, 2.75 \mathrm{mmol})$, and $\mathrm{Ac}_{2} \mathrm{O}(1.08 \mathrm{~mL}, 11.5 \mathrm{mmol})$. After $24 \mathrm{~h}$ at $22{ }^{\circ} \mathrm{C}$, additional pyridine $(0.337 \mathrm{~mL}, 9.16 \mathrm{mmol})$ and $\mathrm{Ac}_{2} \mathrm{O}(1.08 \mathrm{~mL}, 11.5 \mathrm{mmol})$ were added and the solution was stirred for $24 \mathrm{~h}$. ${ }^{1} \mathrm{H}$ NMR spectroscopic analysis of the unpurified product showed a single diastereomer. Purification by flash chromatography (5:95-20:80 EtOAc/hexanes) yielded acetate $\mathbf{5 2}$ as a colorless oil (0.361 g, 63\%): ${ }^{1} \mathrm{H}$ NMR $\left(500 \mathrm{MHz}, \mathrm{CDCl}_{3}\right) \square 7.37-7.31(\mathrm{~m}, 4 \mathrm{H}), 7.29-7.25(\mathrm{~m}, 1 \mathrm{H}), 5.98(\mathrm{~d}, J=$ $2.9,1 \mathrm{H}), 4.52(\mathrm{~d}, J=11.9,1 \mathrm{H}), 4.47(\mathrm{~d}, J=11.9,1 \mathrm{H}), 4.21(\mathrm{~m}, 1 \mathrm{H}), 4.06(\mathrm{dd}, J=9.4,5.2,1 \mathrm{H}), 3.96(\mathrm{dd}, J=$ 9.4, 3.8, 1H), $2.41(\mathrm{~m}, 1 \mathrm{H}), 2.03(\mathrm{~s}, 3 \mathrm{H}), 1.14(\mathrm{~d}, J=7.3,3 \mathrm{H}) ;{ }^{13} \mathrm{C}$ NMR $\left(125 \mathrm{MHz}, \mathrm{CDCl}_{3}\right) \mathrm{1}$ 170.2, 137.7, 128.2, 127.5, 127.2, 103.8, 78.5, 71.6, 71.2, 42.4, 21.0, 10.0; IR (thin film) 2936, 2880, 1738, $1009 \mathrm{~cm}^{-1}$; HRMS (FAB) $m / z$ calcd for $\mathrm{C}_{14} \mathrm{H}_{18} \mathrm{O}_{4} \mathrm{Na}(\mathrm{M}+\mathrm{Na})^{+} 273.1103$, found 273.1102. Anal. Calcd for $\mathrm{C}_{14} \mathrm{H}_{18} \mathrm{O}_{4}: \mathrm{C}$, 67.18; H, 7.25. Found: C, 67.28; H, 7.18.

\section{Allyltrimethylsilane addition to tetrahydrofuran acetates}

General Procedure for Allylation of G-Lactol Acetates: A solution of acetate in $\mathrm{CH}_{2} \mathrm{Cl}_{2}(0.10 \mathrm{M})$ was treated with allyltrimethylsilane (4 equiv) and then cooled to $-78{ }^{\circ} \mathrm{C}$. After the addition of Lewis acid (1.1 equiv), the reaction mixture was warmed to $22{ }^{\circ} \mathrm{C}$ over $2 \mathrm{~h}$. The reaction mixture was treated with saturated aqueous $\mathrm{Na}_{2} \mathrm{HPO}_{4}\left(1 \mathrm{~mL}\right.$ per mmol of acetate). The aqueous layer was then extracted three times with $\mathrm{CH}_{2} \mathrm{Cl}_{2}$ $\left(1 \mathrm{~mL}\right.$ per mmol of acetate), and the organic phases were dried $\left(\mathrm{Na}_{2} \mathrm{SO}_{4}\right)$ and concentrated in vacuo.
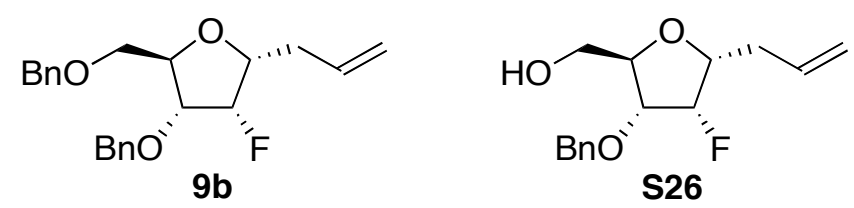

1-Allyl-3,5-di- $\boldsymbol{O}$-benzyl-2-fluoro-2-deoxyribose $(\mathbf{9 b})$. The standard allylation procedure was followed with acetate $8 \mathbf{b}(0.102 \mathrm{~g}, 0.272 \mathrm{mmol})$ and $\mathrm{TiCl}_{4}(0.36 \mathrm{~mL}, 0.33 \mathrm{mmol})$. GC and ${ }^{1} \mathrm{H}$ NMR spectroscopic analysis of the unpurified product showed $\mathbf{9 b}$ as a pair of diastereomers in an 87:13 ratio of 1,3-cis:trans diastereomers and the monobenzylated alkene S26. Purification by flash chromatography (10:90 - 20:80 - 30:70 EtOAc/hexanes) provided benzylated alkene $\mathbf{9 b}$ as a colorless oil $(0.030 \mathrm{~g}, 31 \%)$ and monobenzylated alkene $\mathbf{S 2 6}$ as a colorless oil $(0.036 \mathrm{~g}, 50 \%)$. ${ }^{1} \mathrm{H}$ and ${ }^{13} \mathrm{C}$ NMR spectroscopic analysis of $\mathbf{S 2 6}$ showed a single diastereomer. Benzylation of $\mathbf{S 2 6}$ under standard conditions afforded material that was identical to the major isomer of $\mathbf{9 b}$ by ${ }^{1} \mathrm{H}$ NMR spectroscopic analysis. The overall selectivity of the allylation was calculated to be 93:7.

Major 1,3-cis isomer (9b): ${ }^{1} \mathrm{H}$ NMR $\left(500 \mathrm{MHz}, \mathrm{CDCl}_{3}\right) \square 7.37-7.24(\mathrm{~m}, 10 \mathrm{H}), 5.81(\mathrm{~m}, 1 \mathrm{H}), 5.11(\mathrm{~m}, 2 \mathrm{H})$, $4.89(\mathrm{dt}, J=55.0,2.8,1 \mathrm{H}), 4.69(\mathrm{~d}, J=11.7,1 \mathrm{H}), 4.59(\mathrm{~d}, J=12.1,1 \mathrm{H}), 4.52(\mathrm{~d}, J=11.8,1 \mathrm{H}), 4.49(\mathrm{~d}, J=$ $12.1,1 \mathrm{H}), 4.10(\mathrm{~m}, 3 \mathrm{H}), 3.71(\mathrm{dd}, J=10.8,2.3,1 \mathrm{H}), 3.55(\mathrm{dd}, J=10.8,3.3,1 \mathrm{H}), 2.48(\mathrm{~m}, 2 \mathrm{H}) ;{ }^{13} \mathrm{C}$ NMR $(125$ $\left.\mathrm{MHz}, \mathrm{CDCl}_{3}\right) \square 138.1,137.4,133.5,128.5,128.3,128.0,127.9,127.64,127.58,117.8,89.9$ (d, $\left.J=191\right), 79.9$ (d, $J=18.3$ ), 79.1, 78.5 (d, $J=16.5$ ), 73.4, 72.4, 69.5, 33.6; HRMS (GC-MS, EI) $m / z$ calcd for $\mathrm{C}_{15} \mathrm{H}_{18} \mathrm{FO}_{3}(\mathrm{M}$ - Bn) ${ }^{+}$265.1240, found 265.1236. Anal. Calcd for $\mathrm{C}_{22} \mathrm{H}_{25} \mathrm{FO}_{3}$ : C, 74.13; H, 7.07. Found: C, 74.21; H, 7.08.

Monobenzylated alkene (S26): ${ }^{1} \mathrm{H}$ NMR $\left(500 \mathrm{MHz}, \mathrm{CDCl}_{3}\right) \square 7.38-7.28(\mathrm{~m}, 5 \mathrm{H}), 5.80(\mathrm{~m}, 1 \mathrm{H}), 5.28(\mathrm{dd}, J=$ $17.2,1.5,1 \mathrm{H}), 5.11(\mathrm{dd}, J=10.2,1.2,1 \mathrm{H}), 4.92(\mathrm{dt}, J=55.0,2.6,1 \mathrm{H}), 4.74(\mathrm{~d}, J=11.6,1 \mathrm{H}), 4.59(\mathrm{~d}, J=11.6$, $1 \mathrm{H}), 4.06(\mathrm{~m}, 3 \mathrm{H}), 3.89(\mathrm{~d}, J=12.9,1 \mathrm{H}), 3.62(\mathrm{~m}, 1 \mathrm{H}), 2.48(\mathrm{~m}, 2 \mathrm{H}), 1.80(\mathrm{~s}, \mathrm{br}, 1 \mathrm{H}) ;{ }^{13} \mathrm{C} \mathrm{NMR}(125 \mathrm{MHz}$, $\left.\mathrm{CDCl}_{3}\right) \square$ 137.3, 133.4, 128.5, 128.1, 127.9, 118.0, $90.0(\mathrm{~d}, J=191), 80.2(\mathrm{~d}, J=18), 79.6,78.8$ (d, $\left.J=17\right)$, 72.5, 61.6, 33.8; HRMS (GC-MS, EI) $m / z$ calcd for $\mathrm{C}_{15} \mathrm{H}_{18} \mathrm{FO}_{3}(\mathrm{M}-\mathrm{H})^{+} 265.1240$, found 265.1237. 


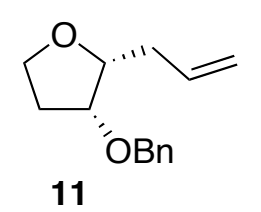

$\left(1 R^{*}, 2 R^{*}\right)$-1-Allyl-2-benzyloxytetrahydrofuran $(11)$. The standard allylation procedure was followed with acetate 10 (2.776 g, $11.75 \mathrm{mmol})$ and $\mathrm{SnBr}_{4}\left(14 \mathrm{~mL}, 14 \mathrm{mmol}, 1.0 \mathrm{M}\right.$ in $\left.\mathrm{CH}_{2} \mathrm{Cl}_{2}\right)$. GC analysis of the unpurified product showed a pair of diastereomers in an 85:15 ratio of 1,2-cis:trans diastereomers. Purification by flash chromatography (5:95 - 30:70 EtOAc/hexanes) provided pure samples of the 1,2-cis and 1,2-trans diastereomers of $\mathbf{1 1}$ as colorless oils $(1.27 \mathrm{~g}, 74 \%)$.

Major 1,2-cis isomer: ${ }^{1} \mathrm{H}$ NMR (500 MHz, $\left.\mathrm{CDCl}_{3}\right) \square 7.34-7.33(\mathrm{~m}, 4 \mathrm{H}), 7.28-7.23(\mathrm{~m}, 1 \mathrm{H}), 5.86(\mathrm{~m}, 1 \mathrm{H}), 5.14$ $(\mathrm{d}, J=17.3,1 \mathrm{H}), 5.05(\mathrm{~d}, J=10.2,1 \mathrm{H}), 4.60(\mathrm{~d}, J=12.0,1 \mathrm{H}), 4.42(\mathrm{~d}, J=12.0,1 \mathrm{H}), 4.02(\mathrm{~m}, 2 \mathrm{H}), 3.76(\mathrm{~m}$, 2H), $2.49(\mathrm{t}, J=6.9,2 \mathrm{H}), 2.05(\mathrm{~m}, 2 \mathrm{H}) ;{ }^{13} \mathrm{C} \mathrm{NMR}\left(125 \mathrm{MHz}, \mathrm{CDCl}_{3}\right) \square 138.3,135.4,128.2,127.44,127.38$, 116.5, 81.7, 78.7, 71.0, 65.9, 33.7, 31.7; IR (thin film) 3069, 2976, 2867, 1641, $1070 \mathrm{~cm}^{-1}$; HRMS (GC-MS, EI) $m / z$ calcd for $\mathrm{C}_{11} \mathrm{H}_{13} \mathrm{O}_{2}\left(\mathrm{M}-\mathrm{C}_{3} \mathrm{H}_{5}\right)^{+}$177.0916, found 177.0914. Anal. Calcd for $\mathrm{C}_{14} \mathrm{H}_{18} \mathrm{O}_{2}$ : C, 77.03; $\mathrm{H}$, 8.31. Found: C, 76.96; H, 8.26.

Minor 1,2-trans isomer: ${ }^{1} \mathrm{H}$ NMR (500 MHz, $\left.\mathrm{CDCl}_{3}\right) \square 7.36-7.11(\mathrm{~m}, 5 \mathrm{H}), 5.82(\mathrm{~m}, 1 \mathrm{H}), 5.07(\mathrm{~m}, 2 \mathrm{H}), 4.52$ $(\mathrm{d}, J=11.9,1 \mathrm{H}), 4.47(\mathrm{~d}, J=11.9,1 \mathrm{H}), 3.85(\mathrm{~m}, 4 \mathrm{H}), 2.26(\mathrm{~m}, 2 \mathrm{H}), 2.00(\mathrm{~m}, 2 \mathrm{H}) ;{ }^{13} \mathrm{C}$ NMR $\left(125 \mathrm{MHz}, \mathrm{CDCl}_{3}\right)$ Q 138.1, 134.4, 128.3, 127.6, 117.1, 83.1, 82.5, 71.2, 66.9, 38.2, 32.1; HRMS (GC-MS, EI) $\mathrm{m} / \mathrm{z}$ calcd for $\mathrm{C}_{11} \mathrm{H}_{13} \mathrm{O}_{2}\left(\mathrm{M}-\mathrm{C}_{3} \mathrm{H}_{5}\right)^{+}$177.0916, found 177.0923.

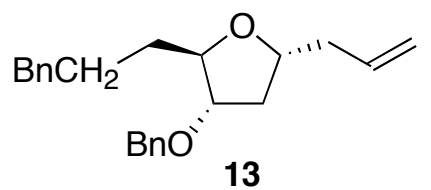

$\left(1 R^{*}, 3 S^{*}, 4 R^{*}\right)$-1-Allyl-3-benzyloxy-4-(3-phenylpropyl)tetrahydrofuran (13). The standard allylation procedure was followed with acetate $12(0.058 \mathrm{~g}, 0.16 \mathrm{mmol})$. GC analysis of the unpurified product showed a single diastereomer. Purification by flash chromatography (4:96 EtOAc/hexanes) provided $\mathbf{1 3}$ as a colorless oil (0.052 g, 94\%): ${ }^{1} \mathrm{H}$ NMR (500 MHz, $\left.\mathrm{CDCl}_{3}\right) \square 7.35-7.27(\mathrm{~m}, 8 \mathrm{H}), 7.25-7.15(\mathrm{~m}, 2 \mathrm{H}), 5.79(\mathrm{~m}, 1 \mathrm{H}), 5.07(\mathrm{~m}$, $2 \mathrm{H}), 4.51(\mathrm{~d}, J=11.8,1 \mathrm{H}), 4.43(\mathrm{~d}, J=11.9,1 \mathrm{H}), 4.04(\mathrm{~m}, 1 \mathrm{H}), 3.98(\mathrm{~m}, 1 \mathrm{H}), 3.78(\mathrm{~m}, 1 \mathrm{H}), 2.61(\mathrm{t}, J=7.7$, 2H), $2.46(\mathrm{~m}, 1 \mathrm{H}), 2.31(\mathrm{~m}, 1 \mathrm{H}), 2.23(\mathrm{~m}, 1 \mathrm{H}), 1.72(\mathrm{~m}, 3 \mathrm{H}), 1.52(\mathrm{~m}, 2 \mathrm{H}) ;{ }^{13} \mathrm{C}$ NMR $\left(125 \mathrm{MHz}, \mathrm{CDCl}_{3}\right) \square$ 142.3, 138.1, 134.9, 128.40, 128.36, 128.2, 127.61, 127.57, 125.7, 116.9, 83.4, 82.4, 76.9, 71.4, 40.4, 37.0, 35.8, 33.2, 27.6; IR (thin film) 2934, 2860, 1454, $1094 \mathrm{~cm}^{-1}$; HRMS (EI) $\mathrm{m} / z$ calcd for $\mathrm{C}_{20} \mathrm{H}_{23} \mathrm{O}_{2}\left(\mathrm{M}-\mathrm{C}_{3} \mathrm{H}_{5}\right)^{+}$ 295.1698, found 295.1693. Anal. Calcd for $\mathrm{C}_{23} \mathrm{H}_{28} \mathrm{O}_{2}: \mathrm{C}, 82.10 ; \mathrm{H}, 8.39$. Found: C, 82.00; H, 8.40.

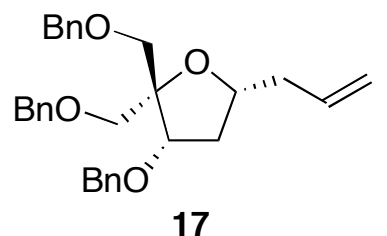

1-Allyl-3,5',5-tri-O-benzyl-2-deoxy-4-(hydroxymethyl)ribose (17). The standard allylation procedure was followed with acetate $16(0.064 \mathrm{~g}, 0.13 \mathrm{mmol})$ and $\mathrm{SnBr}_{4}$. Although the diastereomers were inseparable by GC, HPLC $\left(\mathrm{SiO}_{2}, 3: 97 \mathrm{EtOAc} /\right.$ hexanes$)$ analysis of the unpurified product showed a pair of diastereomers in a 90:10 ratio. Purification by flash chromatography (4:96 EtOAc/hexanes) provided the product as a colorless oil $(0.057 \mathrm{~g}, 93 \%)$. The two diastereomers were separable in small amounts with careful chromatography. 
Elemental analysis, HRMS, and IR data were obtained for $\mathbf{1 7}$ as a mixture of diastereomers: IR (thin film) 2919, 2860, 1453, $1098 \mathrm{~cm}^{-1}$; HRMS (FAB) $\mathrm{m} / z$ calcd for $\mathrm{C}_{30} \mathrm{H}_{35} \mathrm{O}_{4}(\mathrm{M}+\mathrm{H})^{+} 459.2535$, found 459.2538. Anal. Calcd for $\mathrm{C}_{30} \mathrm{H}_{34} \mathrm{O}_{4}$ : C, 78.57; H, 7.47. Found: C, 78.33; H, 7.41.

Major 1,3-cis isomer: ${ }^{1} \mathrm{H}$ NMR $\left(500 \mathrm{MHz}, \mathrm{CDCl}_{3}\right) \square 7.32-7.23(\mathrm{~m}, 15 \mathrm{H}), 5.79(\mathrm{~m}, 1 \mathrm{H}), 5.03(\mathrm{~m}, 2 \mathrm{H}), 4.54(\mathrm{~m}$, $6 \mathrm{H}), 4.18(\mathrm{t}, J=6.5,1 \mathrm{H}), 4.06(\mathrm{~m}, 1 \mathrm{H}), 3.75(\mathrm{~d}, J=10.1,1 \mathrm{H}), 3.65(\mathrm{~d}, J=9.8,1 \mathrm{H}), 3.62(\mathrm{~d}, J=10.2,1 \mathrm{H}), 3.47$ $(\mathrm{d}, J=9.7,1 \mathrm{H}), 2.47(\mathrm{~m}, 1 \mathrm{H}), 2.32(\mathrm{~m}, 1 \mathrm{H}), 2.24(\mathrm{~m}, 1 \mathrm{H}), 1.82(\mathrm{~m}, 1 \mathrm{H}) ;{ }^{13} \mathrm{C}$ NMR $\left(125 \mathrm{MHz}, \mathrm{CDCl}_{3}\right) \mathrm{D} 138.8$, $138.5,134.8,128.24,128.15,127.6,127.5,127.42,127.39,127.3,127.2,116.9,85.4,81.7,76.8,73.5,71.9$, 71.7, 70.8, 40.7, 37.2; ${ }^{13} \mathrm{C}$ NMR $\left(125 \mathrm{MHz}, \mathrm{CD}_{2} \mathrm{Cl}_{2}\right) \square 139.3,139.1,135.5,128.59,128.56,128.5,127.9,127.8$, 127.6, 116.9, 85.7, 82.2, 77.0, 73.83, 73.81, 72.2, 72.1, 71.3, 41.1, 37.6.

Minor 1,3-trans isomer: ${ }^{1} \mathrm{H}$ NMR $\left(500 \mathrm{MHz}, \mathrm{CDCl}_{3}\right) \square 7.33-7.24(\mathrm{~m}, 15 \mathrm{H}), 5.79(\mathrm{~m}, 1 \mathrm{H}), 5.04(\mathrm{~m}, 2 \mathrm{H}), 4.65$ $(\mathrm{d}, J=12.3,1 \mathrm{H}), 4.53(\mathrm{~m}, 4 \mathrm{H}), 4.43(\mathrm{~d}, J=12.0,1 \mathrm{H}), 4.26(\mathrm{~m}, 1 \mathrm{H}), 4.12(\mathrm{dd}, J=5.6,2.0,1 \mathrm{H}), 3.71(\mathrm{~m}, 3 \mathrm{H})$, $3.34(\mathrm{~d}, J=9.6,1 \mathrm{H}), 2.45(\mathrm{~m}, 1 \mathrm{H}), 2.26(\mathrm{~m}, 1 \mathrm{H}), 2.05(\mathrm{~m}, 1 \mathrm{H}), 1.75(\mathrm{~m}, 1 \mathrm{H}) ;{ }^{13} \mathrm{C} \mathrm{NMR}\left(125 \mathrm{MHz}, \mathrm{CDCl}_{3}\right) \square$ 138.7, 138.6, 138.5, 134.5, 128.3, 128.23, 128.17, 128.1, 127.6, 127.42, 127.36, 127.3, 117.0, 86.6, 82.1, 77.1, 73.49, 73.45, 72.1, 71.8, 69.5, 39.9, 36.9; ${ }^{13} \mathrm{C}$ NMR $\left(125 \mathrm{MHz}, \mathrm{CD}_{2} \mathrm{Cl}_{2}\right) \square 139.6,139.4,139.3,135.5,128.8$, $128.7,128.1,128.0,127.9,127.8,117.1,87.2,82.9,77.7,74.03,73.99,72.8,72.4,70.3,40.6,37.5,30.3$.

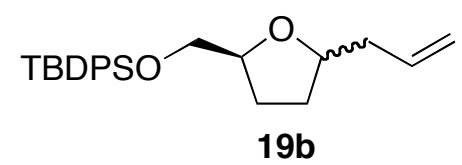

(4S)-1-Allyl-4-t-butyldiphenylsiloxymethyl)tetrahydrofuran (19b). The standard allylation procedure was followed with acetate $18 \mathrm{~b}(0.150 \mathrm{~g}, 0.375 \mathrm{mmol})$ and $\mathrm{SnBr}_{4}\left(0.45 \mathrm{~mL}, 0.45 \mathrm{mmol}, 1.0 \mathrm{M}\right.$ in $\left.\mathrm{CH}_{2} \mathrm{Cl}_{2}\right)$. ${ }^{1} \mathrm{H} \mathrm{NMR}$ spectroscopic analysis of the unpurified product showed a pair of diastereomers in a 60:40 ratio of diastereomers. Purification by flash chromatography (5:95 EtOAc/hexanes) provided the product as a colorless oil $(0.120 \mathrm{~g}, 84 \%)$. The purified product was characterized as a mixture of diastereomers: ${ }^{1} \mathrm{H}$ NMR $(500 \mathrm{MHz}$, $\left.\mathrm{CDCl}_{3}\right) \square 7.77-7.72(\mathrm{~m}, 4 \mathrm{H}), 7.47-7.39(\mathrm{~m}, 6 \mathrm{H}), 5.87(\mathrm{ddt}, J=17.2,10.2,7.0,1 \mathrm{H}), 5.17(\mathrm{~m}, 2 \mathrm{H}), 4.20(\mathrm{~m}$, $0.6 \mathrm{H}), 4.07(\mathrm{~m}, 1 \mathrm{H}), 3.98(\mathrm{~m}, 0.4 \mathrm{H}), 3.70(\mathrm{~m}, 2 \mathrm{H}), 2.39(\mathrm{~m}, 1 \mathrm{H}), 2.28(\mathrm{~m}, 1 \mathrm{H}), 2.05(\mathrm{~m}, 1 \mathrm{H}), 1.92(\mathrm{~m}, 2 \mathrm{H}), 1.59$ $(\mathrm{m}, 1 \mathrm{H}), 1.110(\mathrm{~s}, 3.6 \mathrm{H}), 1.106(\mathrm{~s}, 5.4 \mathrm{H}) ;{ }^{13} \mathrm{C}$ NMR $\left(125 \mathrm{MHz}, \mathrm{CDCl}_{3}\right) \mathrm{1}$ 135.6, 135.2, 135.1, 133.68, 133.67, 133.64, 129.53, 129.51, 127.6, 116.6, 79.4, 79.2, 79.1, 78.7, 66.5, 66.3, 40.3, 40.2, 31.3, 30.5, 27.9, 27.7, 26.8, 19.2; IR (thin film) 3071, 2930, $1642 \mathrm{~cm}^{-1}$; HRMS (CI/isobutane) $\mathrm{m} / z$ calcd for $\mathrm{C}_{20} \mathrm{H}_{23} \mathrm{O}_{2} \mathrm{Si}\left(\mathrm{M}-\mathrm{C}_{4} \mathrm{H}_{9}\right)^{+}$ 323.1467, found 323.1465. Anal. Calcd for $\mathrm{C}_{24} \mathrm{H}_{32} \mathrm{O}_{2} \mathrm{Si}: \mathrm{C}, 75.74 ; \mathrm{H}, 8.47$. Found: $\mathrm{C}, 75.47 ; \mathrm{H}, 8.58$.

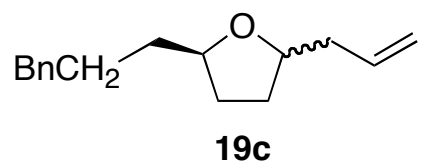

(4R*)-1-Allyl-4-(3-phenylpropyl)tetrahydrofuran (19c). The standard allylation procedure was followed with acetate $18 \mathrm{c}(0.11 \mathrm{~g}, 0.44 \mathrm{mmol})$ and $\mathrm{SnBr}_{4}\left(0.62 \mathrm{~mL}, 0.62 \mathrm{mmol}, 1.0 \mathrm{M}\right.$ in $\left.\mathrm{CH}_{2} \mathrm{Cl}_{2}\right)$. GC analysis of the unpurified product showed a pair of diastereomers in a 57:43 ratio. Purification by flash chromatography (0:100 - 10:90 EtOAc/hexanes) provided the product as a colorless oil $(0.093 \mathrm{~g}, 91 \%)$. The purified product was characterized as a mixture of diastereomers: ${ }^{1} \mathrm{H}$ NMR $\left(500 \mathrm{MHz}, \mathrm{CDCl}_{3}\right) \square 7.31-7.25(\mathrm{~m}, 2 \mathrm{H}), 7.17(\mathrm{~m}$, $3 \mathrm{H}), 5.81(\mathrm{~m}, 1 \mathrm{H}), 5.08(\mathrm{~m}, 1 \mathrm{H}), 5.04(\mathrm{~m}, 1 \mathrm{H}), 3.98(\mathrm{~m}, 1 \mathrm{H}), 3.87$ (quintet, $J=6.5,0.5 \mathrm{H}), 3.81(\mathrm{~m}, 0.5 \mathrm{H}), 2.64$ $(\mathrm{m}, 2 \mathrm{H}), 2.35(\mathrm{~m}, 1 \mathrm{H}), 2.21(\mathrm{~m}, 1 \mathrm{H}), 1.99(\mathrm{~m}, 1 \mathrm{H}), 1.91(\mathrm{~m}, 1 \mathrm{H}), 1.73(\mathrm{~m}, 1 \mathrm{H}), 1.64(\mathrm{~m}, 2 \mathrm{H}), 1.50(\mathrm{~m}, 3 \mathrm{H}) ;{ }^{13} \mathrm{C}$ $\operatorname{NMR}\left(125 \mathrm{MHz}, \mathrm{CDCl}_{3}\right) \square 142.5,135.1,128.4,128.2,125.6,116.64,116.61,79.3,78.7,78.4,77.8,40.4,40.3$, 35.9, 35.5, 31.9, 31.4, 30.9, 30.4, 27.93, 27.88; IR (thin film) 3076, 2860, 1642, $1085 \mathrm{~cm}^{-1}$; HRMS (GC-MS, EI) $m / z$ calcd for $\mathrm{C}_{13} \mathrm{H}_{17} \mathrm{O}\left(\mathrm{M}-\mathrm{C}_{3} \mathrm{H}_{5}\right)^{+}$189.1279, found 189.1279. Anal. Calcd for $\mathrm{C}_{16} \mathrm{H}_{22} \mathrm{O}: \mathrm{C}, 83.43 ; \mathrm{H}, 9.63$. Found: C, 83.14; H, 9.57. 


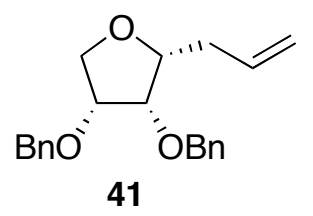

$(1 R, 2 S, 3 R)$-1-Allyl-2,3-dibenzyloxytetrahydrofuran (41). The standard allylation procedure was followed with acetate $40(0.032 \mathrm{~g}, 0.093 \mathrm{mmol})$ and $\mathrm{SnBr}_{4}\left(0.11 \mathrm{~mL}, 0.11 \mathrm{mmol}, 1.0 \mathrm{M}\right.$ in $\left.\mathrm{CH}_{2} \mathrm{Cl}_{2}\right)$. GC and ${ }^{1} \mathrm{H} \mathrm{NMR}$ spectroscopic analysis of the unpurified product showed a pair of diastereomers in a 93:7 ratio of 1,3-cis:trans diastereomers. Purification by flash chromatography (2:98 EtOAc/hexanes) provided the product as a colorless oil $(0.028 \mathrm{~g}, 93 \%):{ }^{1} \mathrm{H}$ NMR $\left(500 \mathrm{MHz}, \mathrm{CDCl}_{3}\right) \square 7.40-7.28(\mathrm{~m}, 10 \mathrm{H}), 5.83$ (ddt, $\left.J=17.2,10.2,6.9,1 \mathrm{H}\right), 5.12$ $(\mathrm{m}, 1 \mathrm{H}), 5.06(\mathrm{~m}, 1 \mathrm{H}), 4.86(\mathrm{~d}, J=11.8,1 \mathrm{H}), 4.62(\mathrm{~m}, 3 \mathrm{H}), 4.17(\mathrm{td}, J=6.4,4.3,1 \mathrm{H}), 3.96(\mathrm{~m}, 4 \mathrm{H}), 2.51(\mathrm{~m}$, $2 \mathrm{H}) ;{ }^{13} \mathrm{C}$ NMR $\left(125 \mathrm{MHz}, \mathrm{CDCl}_{3}\right) \square 138.6,138.3,135.5,128.6,128.5,128.0,127.82,127.78,116.9,80.1,79.3$, 78.2, 73.5, 72.6, 69.3, 34.6; IR (thin film) 3065, 2872, 1641, 1496, $1152 \mathrm{~cm}^{-1}$; HRMS (EI) $\mathrm{m} / \mathrm{z}$ calcd for $\mathrm{C}_{21} \mathrm{H}_{24} \mathrm{O}_{3} \mathrm{Na}(\mathrm{M}+\mathrm{Na})^{+}$347.1623, found 347.1631. Anal. Calcd for $\mathrm{C}_{21} \mathrm{H}_{24} \mathrm{O}_{3}: \mathrm{C}, 77.75 ; \mathrm{H}, 7.46$. Found: $\mathrm{C}$, $77.73 ; \mathrm{H}, 7.45$.

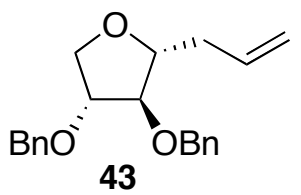

$(1 R, 2 R, 3 R)$-1-Allyl-2,3-dibenzyloxytetrahydrofuran (43). The standard allylation procedure was followed with acetate $42(0.196 \mathrm{~g}, 0.572 \mathrm{mmol})$ and $\mathrm{SnBr}_{4}\left(0.68 \mathrm{~mL}, 0.68 \mathrm{mmol}, 1.0 \mathrm{M}\right.$ in $\left.\mathrm{CH}_{2} \mathrm{Cl}_{2}\right)$. GC and ${ }^{1} \mathrm{H}$ NMR spectroscopic analysis of the unpurified product showed a pair of diastereomers in a 61:39 ratio of 1,3-cis:trans diastereomers. Purification by flash chromatography (5:95 - 10:90 EtOAc/hexanes) provided the product as a colorless oil $(0.163 \mathrm{~g}, 88 \%)$. The purified product was characterized as a mixture of diastereomers: ${ }^{1} \mathrm{H}$ NMR $\left(500 \mathrm{MHz}, \mathrm{CDCl}_{3}\right) \square 7.33-7.41(\mathrm{~m}, 17 \mathrm{H}), 5.90(\mathrm{~m}, 1.7 \mathrm{H}), 5.13(\mathrm{~m}, 3.4 \mathrm{H}), 4.63(\mathrm{~d}, J=11.9,0.7 \mathrm{H}), 4.53(\mathrm{~m}$, $6.1 \mathrm{H}), 4.22(\mathrm{~d}, J=9.8,5.1,0.7 \mathrm{H}), 4.13(\mathrm{~m}, 0.7 \mathrm{H}), 4.10(\mathrm{~m}, 1 \mathrm{H}), 4.06(\mathrm{~m}, 1.7 \mathrm{H}), 3.92(\mathrm{~m}, 2.7 \mathrm{H}), 3.85(\mathrm{~m}, 1 \mathrm{H})$, $3.81(\mathrm{dd}, J=9.8,2.0,0.7 \mathrm{H}), 2.52(\mathrm{~m}, 3.4 \mathrm{H}) ;{ }^{13} \mathrm{C} \mathrm{NMR}\left(125 \mathrm{MHz}, \mathrm{CDCl}_{3}\right) \square 138.1,138.03,137.97,135.3$, $134.7,128.7,128.64,128.62$, 128.61, 128.03, 128.02, 128.00, 127.99, 127.95, 127.9, 127.8, 117.4, 117.0, 87.0, 83.6, 83.2, 82.5, 82.4, 80.5, 72.02, 72.00, 71.6, 71.5, 38.0, 33.4; IR (thin film) 3074, 2970, 2860, 1698, 1426 $\mathrm{cm}^{-1}$; HRMS (EI) $m / z$ calcd for $\mathrm{C}_{21} \mathrm{H}_{25} \mathrm{O}_{3}(\mathrm{M}+\mathrm{H})^{+}$325.1804, found 325.1802. Anal. Calcd for $\mathrm{C}_{21} \mathrm{H}_{24} \mathrm{O}_{3}: \mathrm{C}_{\text {, }}$ 77.75; H, 7.46. Found: C, 77.32; H, 7.43.

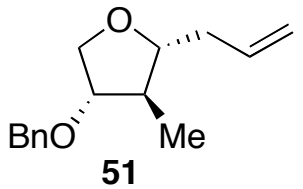

$\left(1 R^{*}, 2 R^{*}, 3 S^{*}\right)$-1-Allyl-3-benzyloxy-2-methyltetrahydrofuran (51). The standard allylation procedure was followed with acetate $\mathbf{5 0}(0.293 \mathrm{~g}, 1.17 \mathrm{mmol})$ and $\mathrm{SnBr}_{4}\left(1.4 \mathrm{~mL}, 1.4 \mathrm{mmol}, 1.0 \mathrm{M}\right.$ in $\left.\mathrm{CH}_{2} \mathrm{Cl}_{2}\right)$. GC analysis of the unpurified product showed a single diastereomer. Purification by flash chromatography (1:99 - 15:85 EtOAc/hexanes) provided the product as a colorless oil $(0.263 \mathrm{~g}, 87 \%):{ }^{1} \mathrm{H}$ NMR $\left(500 \mathrm{MHz} \mathrm{CDCl}_{3}\right) \square$ 7.35-7.30 (m, 4H), 7.30-7.27 (m, 1H), $5.86(\mathrm{ddt}, J=17.2,10.2,7.0,1 \mathrm{H}), 5.12(\mathrm{ddd}, J=17.2,3.4,1.6,1 \mathrm{H})$, $5.07(\mathrm{~m}, 1 \mathrm{H}), 4.50(\mathrm{~s}, 2 \mathrm{H}), 3.91(\mathrm{dd}, J=9.7,3.2,1 \mathrm{H}), 3.80(\mathrm{dd}, J=9.7,5.4,1 \mathrm{H}), 3.74(\mathrm{~m}, 1 \mathrm{H}), 3.43(\mathrm{~m}, 1 \mathrm{H})$, $2.39(\mathrm{~m}, 2 \mathrm{H}), 2.15$ (d of quintet, $J=7.1,4.2,1 \mathrm{H}), 1.06(\mathrm{~d}, J=7.0,3 \mathrm{H}) ;{ }^{13} \mathrm{C} \mathrm{NMR}\left(125 \mathrm{MHz}, \mathrm{CDCl}_{3}\right) \square 138.1$, $134.8,128.3,127.54,127.49,116.8,86.2,84.9,71.4,71.3,44.8,38.6,16.2$; IR (thin film) 3066, 2872, 1642 
$\mathrm{cm}^{-1}$; HRMS (CI/isobutane) $\mathrm{m} / z$ calcd for $\mathrm{C}_{15} \mathrm{H}_{21} \mathrm{O}_{2}(\mathrm{M}+\mathrm{H})^{+}$233.1541, found 233.1544. Anal. Calcd for $\mathrm{C}_{15} \mathrm{H}_{20} \mathrm{O}_{2}: \mathrm{C}, 77.55 ; \mathrm{H}, 8.68$. Found: $\mathrm{C}, 77.30 ; \mathrm{H}, 8.63$.

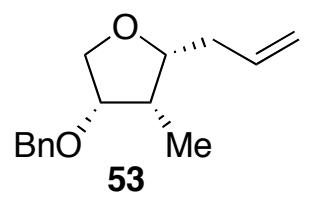

$\left(1 R^{*}, 2 S^{*}, 3 S^{*}\right)$-1-Allyl-3-benzyloxy-2-methyltetrahydrofuran (53). The standard allylation procedure was followed with acetate $\mathbf{5 2}(0.293 \mathrm{~g}, 1.17 \mathrm{mmol})$ and $\mathrm{SnBr}_{4}\left(1.4 \mathrm{~mL}, 1.4 \mathrm{mmol}, 1.0 \mathrm{M}\right.$ in $\left.\mathrm{CH}_{2} \mathrm{Cl}_{2}\right)$. GC analysis of the unpurified product showed a pair of diastereomers in a 98:2 ratio of 1,3-cis:trans diastereomers. Purification by flash chromatography $(1: 99-15: 85 \mathrm{EtOAc} /$ hexanes $)$ provided the product as an oil $(0.238 \mathrm{~g}, 88 \%):{ }^{1} \mathrm{H}$ NMR $\left(500 \mathrm{MHz}, \mathrm{CDCl}_{3}\right) \square 7.36-7.32(\mathrm{~m}, 4 \mathrm{H}), 7.30-7.24(\mathrm{~m}, 1 \mathrm{H}), 5.83(\mathrm{~m}, 1 \mathrm{H}), 5.11(\mathrm{~m}, 1 \mathrm{H}), 5.05(\mathrm{~m}, 1 \mathrm{H}), 4.48(\mathrm{~s}$, $2 \mathrm{H}), 4.11(\mathrm{dd}, J=11.3,5.9,1 \mathrm{H}), 3.93(\mathrm{dt}, J=8.8,5.5,1 \mathrm{H}), 3.87(\mathrm{dd}, J=9.1,6.2,1 \mathrm{H}), 3.82(\mathrm{~m}, 1 \mathrm{H}), 2.39(\mathrm{~m}$, $1 \mathrm{H}), 2.31(\mathrm{~m}, 1 \mathrm{H}), 2.21(\mathrm{~m}, 1 \mathrm{H}), 1.01(\mathrm{~d}, J=7.2,3 \mathrm{H}) ;{ }^{13} \mathrm{C} \mathrm{NMR}\left(125 \mathrm{MHz}, \mathrm{CDCl}_{3}\right) \square$ 138.2, 135.4, 128.2, 127.4, 127.2, 116.3, 80.51, 80.48, 71.7, 70.0, 38.7, 35.6, 7.7; IR (thin film) 3030, 2918, 2878, $1642 \mathrm{~cm}^{-1}$; HRMS (GC-MS, EI) $\mathrm{m} / z$ calcd for $\mathrm{C}_{12} \mathrm{H}_{15} \mathrm{O}_{2}\left(\mathrm{M}-\mathrm{C}_{3} \mathrm{H}_{5}\right)^{+}$191.1072, found 191.1066. Anal. Calcd for $\mathrm{C}_{15} \mathrm{H}_{20} \mathrm{O}_{2}: \mathrm{C}, 77.55 ; \mathrm{H}, 8.68$. Found: $\mathrm{C}, 77.28 ; \mathrm{H}, 8.80$.

\section{Stereochemical proofs of allyl products}

A. The stereochemistry of $\mathbf{9 b}$ was determined by conversion to $\mathbf{S 2 7}$ and the subsequent analysis of nOe data.

Synthetic Scheme.
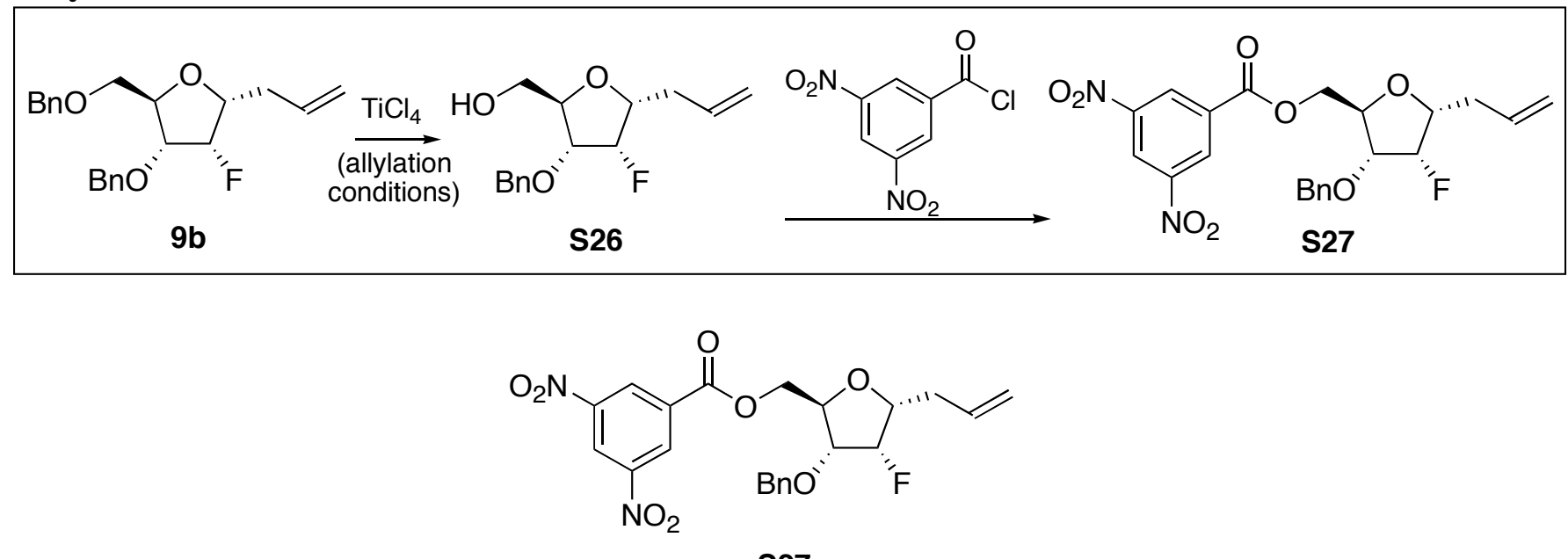

S27

1-Allyl-3-O-Benxyl-5-O-(3,5-dinitrobenzoyl)-2-fluoro-2-deoxyribose (S27). To a solution of S26 (0.030 g, $0.11 \mathrm{mmol})$ in $5 \mathrm{~mL}$ of $\mathrm{CH}_{2} \mathrm{Cl}_{2}$ was added 3,5-dinitrobenzoyl chloride $(0.52 \mathrm{~g}, 0.23 \mathrm{mmol})$ and DMAP $(<0.005$ g). After stirring for $2 \mathrm{~h}$ at $25^{\circ} \mathrm{C}$, the reaction mixture was treated with $10 \mathrm{~mL}$ of saturated aqueous $\mathrm{Na}_{2} \mathrm{HPO}_{4}$. The organic layer was saved and the aqueous layer was extracted with $2 \square 10 \mathrm{~mL}$ of $\mathrm{CH}_{2} \mathrm{Cl}_{2}$. Concentration in vacuo and flash chromatography (10:90 EtOAc/hexanes) yielded the product as a pale yellow oil (0.030 $\mathrm{g}$,

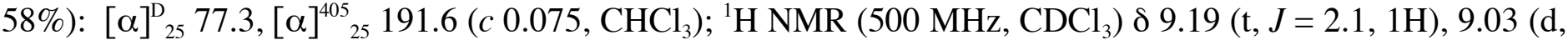
$J=2.0,2 \mathrm{H}), 7.31-7.21(\mathrm{~m}, 5 \mathrm{H}), 5.81(\mathrm{~m}, 1 \mathrm{H}), 5.19(\mathrm{~m}, 2 \mathrm{H}), 5.01(\mathrm{dt}, J=54.6,2.9,1 \mathrm{H}), 4.79(\mathrm{~d}, J=11.8,1 \mathrm{H})$, $4.66(\mathrm{dd}, J=11.7,3.5,1 \mathrm{H}), 4.18(\mathrm{~m}, 2 \mathrm{H}), 4.41(\mathrm{~m}, 1 \mathrm{H}), 4.12(\mathrm{~m}, 1 \mathrm{H}), 3.98(\mathrm{~m}, 1 \mathrm{H}), 2.51(\mathrm{~m}, 2 \mathrm{H}) ;{ }^{13} \mathrm{C}$ NMR $\left(125 \mathrm{MHz}, \mathrm{CDCl}_{3}\right) \square 162.3,148.5,136.8,133.4,132.9,129.4,128.5,128.1,127.9,122.4,118.3,89.1$ (d, $J=$ 
192), 80.1 (d, $J=19), 79.0$ (d, $J=17), 76.6,72.2,66.0,33.5 ;{ }^{19} \mathrm{~F}$ NMR $\left(376 \mathrm{MHz}, \mathrm{CDCl}_{3}\right.$, fluorobenzene external standard) $\square-214.8$ (m); IR (thin film) 3100, 2880, 1732, 1630, 1538, $1462 \mathrm{~cm}^{-1}$; HRMS (CI/isobutane) $m / z$ calcd for $\mathrm{C}_{22} \mathrm{H}_{21} \mathrm{~N}_{2} \mathrm{O}_{8} \mathrm{~F}(\mathrm{M})^{+} 460.1282$, found 460.1273 .

Relevant DPFGSE-nOe data (mixing time $0.5 \mathrm{~s}$ ): (the peaks in the ${ }^{1} \mathrm{H}$ NMR spectra were assigned using ${ }^{1} \mathrm{H} /{ }^{1} \mathrm{H}$ COSY, ${ }^{1} \mathrm{H}$ NMR chemical shifts, and ${ }^{1} \mathrm{H}$ NMR coupling constants)
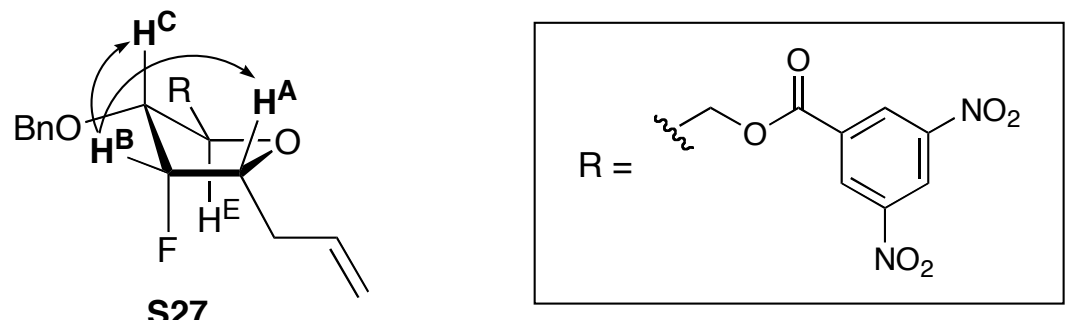

The observation of nOe between $\mathrm{H}^{\mathrm{B}}$ and $\mathrm{H}^{\mathrm{A}}$ along with $\mathrm{H}^{\mathrm{C}}$ suggests a 1,3-cis configuration.

B. The stereochemistry of the major and minor isomer of alkene $\mathbf{1 1}$ was determined by conversion to S31 and S35 and subsequent ${ }^{1} \mathrm{H}$ NMR spectroscopic analysis of each compound.

Synthetic Scheme.

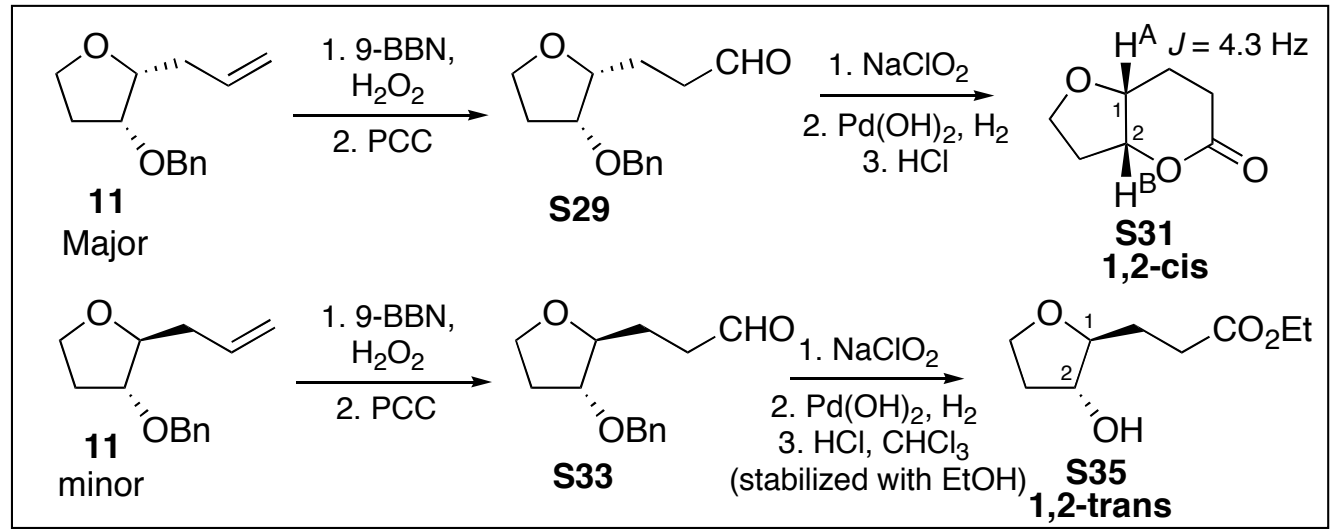

The major isomer of $\mathbf{1 1}$ was isolated and converted to aldehyde $\mathbf{S 2 9}$ over two steps. Oxidation to the corresponding carboxylic acid was followed by removal of the benzyl protecting group. In the presence of acid, cyclization occurred to afford the 6,5-fused lactone S31. The ${ }^{1} \mathrm{H}$ NMR coupling constant of $4.3 \mathrm{~Hz}$ between $\mathrm{H}^{\mathrm{A}}$ and $\mathrm{H}^{\mathrm{B}}$ (consistent with an axial-equatorial coupling constant) ${ }^{26}$ indicates a 1,2 cis-configuration. The spectral data obtained for $\mathbf{S 3 1}$ matched the reported values for this compound. ${ }^{27}$

Subjection of the minor isomer to the similar reaction sequence afforded ester S35. Failure to form the 6,5-fused lactone suggests a 1,2-trans configuration.

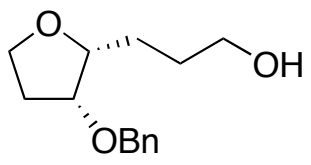


$\left(1 R^{*}, 2 R^{*}\right)-1-(3-H y d r o x y p r o p y l)-2-b e n z y l o x y t e t r a h y d r o f u r a n ~(S 28)$. To a cooled $\left(0{ }^{\circ} \mathrm{C}\right)$ solution of alkene $11(0.500 \mathrm{~g}, 2.29 \mathrm{mmol})$ in THF $(23 \mathrm{~mL})$ was added 9-BBN $(0.700 \mathrm{~g}, 5.73 \mathrm{mmol})$. After $6 \mathrm{~h}$ at $22^{\circ} \mathrm{C}$, the solution was heated to reflux for $1 \mathrm{~h}$, then cooled to $22^{\circ} \mathrm{C}$. $\mathrm{H}_{2} \mathrm{O}_{2}(1.0 \mathrm{~mL}, 30 \%$ aqueous $), \mathrm{NaOH}(3.8 \mathrm{~mL}, 3.0$ $\mathrm{M}$ aqueous), and $\mathrm{MeOH}(15 \mathrm{~mL})$ were added to the reaction mixture and the solution was heated to $40{ }^{\circ} \mathrm{C}$ for 12 h. The mixture was diluted with brine $(150 \mathrm{~mL})$ and extracted with $4 \square 20 \mathrm{~mL}$ of $\mathrm{Et}_{2} \mathrm{O}$. The combined organic phases were concentrated in vacuo, dissolved in $\mathrm{CH}_{2} \mathrm{Cl}_{2}$, filtered through $\mathrm{Na}_{2} \mathrm{SO}_{4}$, and concentrated in vacuo. The resulting oil was purified by flash chromatography (20:80 - 50:50 EtOAc/hexanes) to afford the product as a colorless oil $(0.341 \mathrm{~g}, 63 \%):{ }^{1} \mathrm{H}$ NMR $\left(500 \mathrm{MHz} \mathrm{CDCl}_{3}\right) \square 7.37-7.33(\mathrm{~m}, 4 \mathrm{H}), 7.31-7.27(\mathrm{~m}, 1 \mathrm{H}), 4.62(\mathrm{~d}, J$ $=12.1,1 \mathrm{H}), 4.42(\mathrm{~d}, J=12.1,1 \mathrm{H}), 4.04(\mathrm{dd}, J=15.7,7.7,1 \mathrm{H}), 3.99(\mathrm{~m}, 1 \mathrm{H}), 3.79(\mathrm{td}, J=8.5,5.2,1 \mathrm{H}), 3.72$ $(\mathrm{m}, 1 \mathrm{H}), 3.66(\mathrm{~m}, 2 \mathrm{H}), 2.51(\mathrm{~s}, 1 \mathrm{H}), 2.08(\mathrm{~m}, 2 \mathrm{H}), 1.79(\mathrm{~m}, 2 \mathrm{H}), 1.49(\mathrm{~m}, 2 \mathrm{H}) ;{ }^{13} \mathrm{C} \mathrm{NMR}\left(125 \mathrm{MHz}, \mathrm{CDCl}_{3}\right)$ 138.3, 128.3, 127.54, 127.46, 82.4, 79.1, 71.0, 65.9, 62.8, 31.8, 30.1, 26.1; IR (thin film) 3410, 3062, 2940, 2867, $1065 \mathrm{~cm}^{-1}$; HRMS (CI/isobutane) $m / z$ calcd for $\mathrm{C}_{14} \mathrm{H}_{21} \mathrm{O}_{3}(\mathrm{M}+\mathrm{H})^{+}$237.1490, found 237.1495.

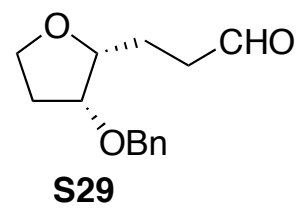

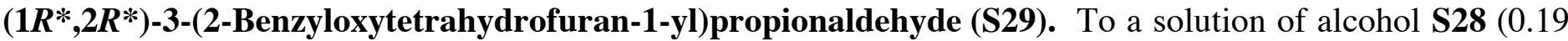
$\mathrm{g}, 0.80 \mathrm{mmol})$ in $\mathrm{CH}_{2} \mathrm{Cl}_{2}(20 \mathrm{~mL})$ was added pyridinium chlorochromate $(0.52 \mathrm{~g}, 2.4 \mathrm{mmol})$. The mixture was stirred vigorously for $4 \mathrm{~h}$. Silica gel $(1 \mathrm{~g})$ was added to the reaction mixture and the solvent was removed in vacuo. The impure material was poured onto a silica gel column and purified by flash chromatography (20:80 50:50 EtOAc/hexanes) to afford the product as a colorless oil $(0.092 \mathrm{~g}, 49 \%):{ }^{1} \mathrm{H} \mathrm{NMR}\left(500 \mathrm{MHz}, \mathrm{CDCl}_{3}\right) \square$ $9.75(\mathrm{t}, J=1.7,1 \mathrm{H}), 7.36-7.32(\mathrm{~m}, 4 \mathrm{H}), 7.30-7.27(\mathrm{~m}, 1 \mathrm{H}), 4.61(\mathrm{~d}, J=12.0,1 \mathrm{H}), 4.41(\mathrm{~d}, J=12.0,1 \mathrm{H}), 3.99$ (m, 2H), $3.74(\mathrm{~m}, 2 \mathrm{H}), 2.54(\mathrm{~m}, 2 \mathrm{H}), 2.04(\mathrm{~m}, 4 \mathrm{H}) ;{ }^{13} \mathrm{C}$ NMR $\left(125 \mathrm{MHz}, \mathrm{CDCl}_{3}\right) \square 202.4,138.1,128.3,127.6$, 127.5, 80.9, 78.8, 71.0, 65.8, 40.8, 31.8, 21.9; IR (thin film) 3063, 2937, 2871, 2724, $1722 \mathrm{~cm}^{-1}$; HRMS (FAB) $m / z$ calcd for $\mathrm{C}_{14} \mathrm{H}_{17} \mathrm{O}_{3}(\mathrm{M}-\mathrm{H})^{+} 233.1176$, found 233.1180 .

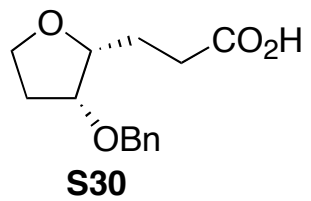

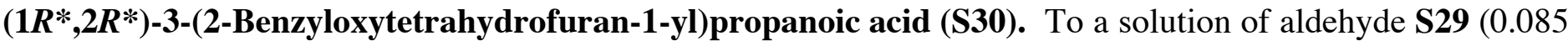
$\mathrm{g}, 0.36 \mathrm{mmol})$ in $t$ - $\mathrm{BuOH}(3.6 \mathrm{~mL})$ were added 2-methyl-2-butene (1.7 mL, $3.4 \mathrm{mmol}, 2.0 \mathrm{M}$ in THF $), \mathrm{NaH}_{2} \mathrm{PO}_{4}$ $\left(0.32 \mathrm{~g}, 2.7 \mathrm{mmol}\right.$, dissolved in $3.6 \mathrm{~mL}$ of $\left.\mathrm{H}_{2} \mathrm{O}\right)$, and $\mathrm{NaClO}_{2}(0.325 \mathrm{~g}, 3.60 \mathrm{mmol})$. The mixture was stirred for $12 \mathrm{~h}$ and concentrated in vacuo. The solution was acidified with $\mathrm{HCl}(1 \mathrm{~mL}, 12 \mathrm{M})$, dissolved in EtOAc (50 $\mathrm{mL})$, washed with $1 \square 20 \mathrm{~mL}$ of brine and concentrated in vacuo. The residue was dissolved in $\mathrm{Et}_{2} \mathrm{O}(50 \mathrm{~mL})$ and washed with $2 \square 20 \mathrm{~mL}$ of $1 \mathrm{M} \mathrm{NaOH}$. The combined aqueous layers were acidified with $\mathrm{HCl}(12 \mathrm{M})$ to $\mathrm{pH}<1$ and extracted with $2 \square 20 \mathrm{~mL}$ of $\mathrm{Et}_{2} \mathrm{O}$. The combined organic layers were dried $\left(\mathrm{Na}_{2} \mathrm{SO}_{4}\right)$ and concentrated in vacuo to provide the product as an oil $(0.070 \mathrm{~g}, 78 \%)$ : ${ }^{1} \mathrm{H} \mathrm{NMR}\left(500 \mathrm{MHz}, \mathrm{CDCl}_{3}\right) \square 10.69$ (br $\mathrm{s}, 1 \mathrm{H}), 7.35-7.15(\mathrm{~m}, 5 \mathrm{H}), 4.60(\mathrm{~d}, J=12.0,1 \mathrm{H}), 4.43(\mathrm{~d}, J=12.0,1 \mathrm{H}), 4.00(\mathrm{~m}, 2 \mathrm{H}), 3.79(\mathrm{~m}, 2 \mathrm{H}), 2.48(\mathrm{~m}$, 2H), $2.04(\mathrm{~m}, 4 \mathrm{H}) ;{ }^{13} \mathrm{C}$ NMR $\left(125 \mathrm{MHz}, \mathrm{CDCl}_{3}\right) \square$ 179.4, 138.1, 128.3, 127.6, 127.4, 80.8, 78.8, 71.1, 65.8, 31.7, 30.8, 24.2; IR (thin film) 3031 (br), 2940 (br), $1710 \mathrm{~cm}^{-1}$; HRMS (CI/isobutane) $\mathrm{m} / z$ calcd for $\mathrm{C}_{14} \mathrm{H}_{19} \mathrm{O}_{4}$ $(\mathrm{M}+\mathrm{H})^{+}$251.1283, found 251.1277. 


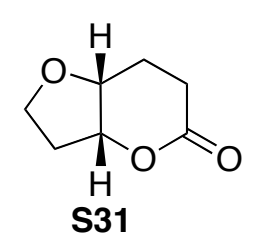

$\left(1 R^{*}, 6 R^{*}\right)$-2,7-dioxabicyclo[4.3.0]nonan-3-one (S31). To a solution of acid $\mathbf{S 3 0}(0.065 \mathrm{~g}, 0.20 \mathrm{mmol})$ in EtOAc $(10 \mathrm{~mL})$ was added $\mathrm{Pd}(\mathrm{OH})_{2}(0.065 \mathrm{~g}$, Degussa type E101, $10 \%$ on activated carbon). The mixture was stirred under an atmosphere of $\mathrm{H}_{2}(50 \mathrm{psi})$ for $12 \mathrm{~h}$. The reaction mixture was filtered through Celite and concentrated in vacuo. The residue was dissolved in $\mathrm{CHCl}_{3}$ (stabilized with $\mathrm{EtOH}$ ), and stirred with $\mathrm{HCl}(0.05$ $\mathrm{mL}, 12 \mathrm{M}$ ) for $30 \mathrm{~min}$. The solution was concentrated in vacuo, dissolved in $\mathrm{CH}_{2} \mathrm{Cl}_{2}$, filtered through $\mathrm{Na}_{2} \mathrm{SO}_{4}$, and concentrated in vacuo to afford the product as a colorless oil. Purification by flash chromatography (50:50 EtOAc/hexane) provided the lactone $\mathbf{S 3 1}(0.039 \mathrm{~g}, 96 \%)$. The spectral data obtained matched the reported values for this compound: ${ }^{27}{ }^{1} \mathrm{H}$ NMR $\left(500 \mathrm{MHz}, \mathrm{CDCl}_{3}\right) \square 4.93$ (ddd, $\left.J=6.1,4.3,2.0,1 \mathrm{H}\right), 4.14$ (dd, $J=8.6$, 4.3, 1H), $4.03(\mathrm{dd}, J=16.0,7.6,1 \mathrm{H}), 3.88(\mathrm{td}, J=8.4,5.0,1 \mathrm{H}), 2.61(\mathrm{~m}, 1 \mathrm{H}), 2.37(\mathrm{~m}, 2 \mathrm{H}), 2.23(\mathrm{~m}, 1 \mathrm{H}), 2.13$ (m, 2H); ${ }^{13} \mathrm{C}$ NMR (125 MHz, $\left.\mathrm{CDCl}_{3}\right) \square$ 171.3, 81.8, 73.8, 66.7, 34.5, 25.4, 23.4; HRMS (CI/ammonia) $\mathrm{m} / \mathrm{z}$ calcd for $\mathrm{C}_{7} \mathrm{H}_{10} \mathrm{O}_{3}(\mathrm{M})^{+}$142.0630, found 142.0634 .

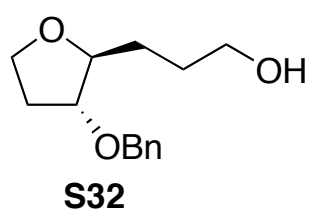

$\left(1 S^{*}, 2 R^{*}\right)$-1-(3-Hydroxypropyl)-2-benzyloxytetrahydrofuran (S32). To a cooled $\left(0{ }^{\circ} \mathrm{C}\right)$ solution of the minor isomer of alkene $11(0.125 \mathrm{~g}, 0.573 \mathrm{mmol})$ in THF $(8 \mathrm{~mL})$ was added 9-BBN $(0.242 \mathrm{~g}, 1.98 \mathrm{mmol})$. After $6 \mathrm{~h}$ at $22{ }^{\circ} \mathrm{C}$, the solution was heated to reflux for $1 \mathrm{~h}$, then cooled to $22{ }^{\circ} \mathrm{C} . \mathrm{H}_{2} \mathrm{O}_{2}(0.5 \mathrm{~mL}, 30 \%$ aqueous), $\mathrm{NaOH}(2.0 \mathrm{~mL}, 3.0 \mathrm{M}$ aqueous), and $\mathrm{MeOH}(5 \mathrm{~mL})$ were added to the mixture and the solution was heated to $40{ }^{\circ} \mathrm{C}$ for $6 \mathrm{~h}$. The mixture was diluted with brine $(100 \mathrm{~mL})$ and extracted with $4 \square 10 \mathrm{~mL}$ of $\mathrm{Et}_{2} \mathrm{O}$. The combined organic phases were concentrated in vacuo, dissolved in $\mathrm{CH}_{2} \mathrm{Cl}_{2}$, filtered through $\mathrm{Na}_{2} \mathrm{SO}_{4}$, and concentrated in vacuo. Purification by flash chromatography (25:75 - 55:45 EtOAc/hexanes) provided the product as a colorless oil $(0.80 \mathrm{~g}, 59 \%):{ }^{1} \mathrm{H}$ NMR $\left(500 \mathrm{MHz}, \mathrm{CDCl}_{3}\right) \square 7.34(\mathrm{~m}, 4 \mathrm{H}), 7.27(\mathrm{~m}, 1 \mathrm{H}), 4.54(\mathrm{~d}, J=$ $11.8,1 \mathrm{H}), 4.48(\mathrm{~d}, J=11.8,1 \mathrm{H}), 3.94(\mathrm{~m}, 1 \mathrm{H}), 3.89(\mathrm{dd}, J=8.0,2.0,1 \mathrm{H}), 3.85(\mathrm{~m}, 1 \mathrm{H}), 3.78(\mathrm{~m}, 1 \mathrm{H}), 3.61(\mathrm{~m}$, 2H), 2.75 (br s, 1H), $2.01(\mathrm{~m}, 2 \mathrm{H}), 1.68(\mathrm{~m}, 3 \mathrm{H}), 1.52(\mathrm{~m}, 1 \mathrm{H}) ;{ }^{13} \mathrm{C}$ NMR $\left(125 \mathrm{MHz}, \mathrm{CDCl}_{3}\right) \mathrm{1}$ 137.9, 128.3, 127.62, 127.56, 83.6, 83.2, 71.3, 66.6, 62.5, 32.0, 30.7, 29.4; IR (thin film) 3399, 3030, 2940, $2867 \mathrm{~cm}^{-1}$; HRMS (CI/ammonia) $m / z$ calcd for $\mathrm{C}_{14} \mathrm{H}_{21} \mathrm{O}_{3}(\mathrm{M}+\mathrm{H})^{+}$237.1491, found 237.1492.

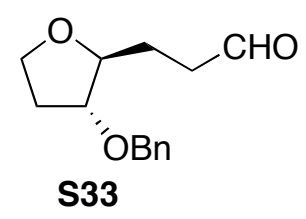

$\left(1 S^{*}, 2 R^{*}\right)$-2-(3-Benzyloxytetrahydrofuran-1-yl)propionaldehyde (S33). To a solution of alcohol S32 (0.080 $\mathrm{g}, 0.34 \mathrm{mmol})$ in $\mathrm{CH}_{2} \mathrm{Cl}_{2}(9 \mathrm{~mL})$ was added pyridinium chlorochromate $(0.293 \mathrm{~g}, 1.36 \mathrm{mmol})$. After the mixture was stirred vigorously for $4 \mathrm{~h}$, silica gel $(1 \mathrm{~g})$ was added to the reaction mixture and the solvent was removed in vacuo. The residue was poured onto a silica gel column and purified by flash chromatography (20:80 - 35:65 EtOAc/hexanes) to afford $\mathbf{S 3 3}$ as a colorless oil $(0.045 \mathrm{~g}, 57 \%):{ }^{1} \mathrm{H}$ NMR $\left(500 \mathrm{MHz}, \mathrm{CDCl}_{3}\right) \square$ $9.76(\mathrm{t}, J=1.4,1 \mathrm{H}), 7.37-7.27(\mathrm{~m}, 5 \mathrm{H}), 4.55(\mathrm{~d}, J=11.8,1 \mathrm{H}), 4.48(\mathrm{~d}, J=11.8,1 \mathrm{H}), 3.91(\mathrm{~m}, 2 \mathrm{H}), 3.84(\mathrm{~m}$, $1 \mathrm{H}), 3.78(\mathrm{~m}, 1 \mathrm{H}), 2.58(\mathrm{~m}, 1 \mathrm{H}), 2.50(\mathrm{~m}, 1 \mathrm{H}), 2.02(\mathrm{~m}, 2 \mathrm{H}), 1.91(\mathrm{~m}, 1 \mathrm{H}), 1.74(\mathrm{~m}, 1 \mathrm{H}) ;{ }^{13} \mathrm{C} \mathrm{NMR}(125 \mathrm{MHz}$, 
$\left.\mathrm{CDCl}_{3}\right) \square 201.8,137.9,128.4,127.7,127.6,83.0,82.7,71.3,66.7,40.4,32.1,26.2$; IR (thin film) 3031, 2972, 2868, 2726, $1723 \mathrm{~cm}^{-1}$; HRMS (CI/ammonia) $\mathrm{m} / \mathrm{z}$ calcd for $\mathrm{C}_{14} \mathrm{H}_{18} \mathrm{O}_{3}(\mathrm{M})^{+}$234.1256, found 234.1253.

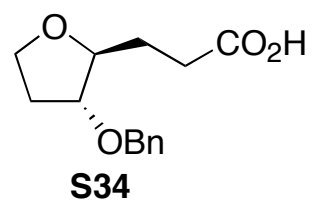

$\left(1 S^{*}, 2 R^{*}\right)$-3-(2-Benzyloxytetrahydrofuran-1-yl)propanoic acid (S34). To a solution of aldehyde $\mathbf{S 3 3}(0.045$ $\mathrm{g}, 0.19 \mathrm{mmol})$ in $t$ - $\mathrm{BuOH}(3.0 \mathrm{~mL})$ were added 2-methyl-2-butene $(0.88 \mathrm{~mL}, 1.8 \mathrm{mmol}, 2.0 \mathrm{M}$ in THF), $\mathrm{NaH}_{2} \mathrm{PO}_{4}\left(0.18 \mathrm{~g}, 2.7 \mathrm{mmol}\right.$, dissolved in $3.0 \mathrm{~mL}$ of $\left.\mathrm{H}_{2} \mathrm{O}\right)$, and $\mathrm{NaClO}_{2}(0.172 \mathrm{~g}, 1.90 \mathrm{mmol})$. The mixture was stirred for $12 \mathrm{~h}$ and concentrated in vacuo. The residue was acidified with $\mathrm{HCl}(0.5 \mathrm{~mL}, 12 \mathrm{M})$, dissolved in EtOAc $(30 \mathrm{~mL})$, washed with $1 \square 10 \mathrm{~mL}$ of brine and concentrated in vacuo. The resultant oil was dissolved in $\mathrm{Et}_{2} \mathrm{O}(30 \mathrm{~mL})$ and washed with $2 \square 10 \mathrm{~mL}$ of $1 \mathrm{M} \mathrm{NaOH}$. The combined aqueous layers were acidified with $\mathrm{HCl}(12 \mathrm{M})$ to $\mathrm{pH}<1$ and extracted with $2 \square 20 \mathrm{~mL}$ of $\mathrm{Et}_{2} \mathrm{O}$. The combined organic layers were dried $\left(\mathrm{Na}_{2} \mathrm{SO}_{4}\right)$ and concentrated in vacuo to provide the product as an oil $(0.039 \mathrm{~g}, 81 \%):{ }^{1} \mathrm{H}$ NMR $\left(500 \mathrm{MHz}, \mathrm{CDCl}_{3}\right)$ 7.36-7.27 (m, 5H), $4.55(\mathrm{~d}, J=11.8,1 \mathrm{H}), 4.49(\mathrm{~d}, J=11.8,1 \mathrm{H}), 3.92(\mathrm{~m}, 3 \mathrm{H}), 3.80(\mathrm{~m}, 1 \mathrm{H}), 2.47(\mathrm{~m}, 2 \mathrm{H}), 2.03$ $(\mathrm{m}, 2 \mathrm{H}), 1.90(\mathrm{~m}, 1 \mathrm{H}), 1.75(\mathrm{~m}, 1 \mathrm{H}) ;{ }^{13} \mathrm{C}$ NMR $\left(125 \mathrm{MHz}, \mathrm{CDCl}_{3}\right) \square 178.4,137.9,128.5,127.8,127.7,83.0$, 82.7, 71.3, 66.8, 32.1, 30.6, 28.6; IR (thin film) 3031 (br), $1709 \mathrm{~cm}^{-1}$; HRMS (FAB) $\mathrm{m} / z$ calcd for $\mathrm{C}_{14} \mathrm{H}_{19} \mathrm{O}_{4}$ (M $+\mathrm{H})^{+} 251.1283$, found 251.1283 .

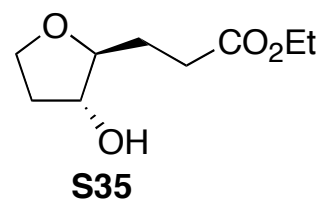

Ethyl (1S*,2R*)-3-(2-hydroxytetrahydrofuran-1-yl)propanoate (S35). To a solution of acid $\mathbf{S 3 4}(0.039 \mathrm{~g}$, $0.16 \mathrm{mmol})$ in EtOAc $(10 \mathrm{~mL})$ was added $\mathrm{Pd}(\mathrm{OH})_{2}(0.025 \mathrm{~g}$, Degussa type E101, $10 \%$ on activated carbon). The mixture was stirred under an atmosphere of $\mathrm{H}_{2}(50 \mathrm{psi})$ for $12 \mathrm{~h}$. The reaction mixture was filtered through Celite, concentrated in vacuo, dissolved in $\mathrm{CHCl}_{3}$ (stabilized with EtOH), and stirred with $\mathrm{HCl}(0.05 \mathrm{~mL}, 12 \mathrm{M})$ for $36 \mathrm{~h}$. The solution was concentrated in vacuo, dissolved in $\mathrm{CH}_{2} \mathrm{Cl}_{2}$, filtered through $\mathrm{Na}_{2} \mathrm{SO}_{4}$ and concentrated in vacuo to afford the product as a solid. Purification by flash chromatography (50:50 EtOAc/hexanes) provided the product as a white solid $(0.013 \mathrm{~g}, 43 \%):{ }^{1} \mathrm{H}$ NMR $\left(500 \mathrm{MHz}, \mathrm{CDCl}_{3}\right) \square 4.14$ (q, $J$ $=7.1,2 \mathrm{H}), 4.09(\mathrm{dt}, J=6.4,3.2,1 \mathrm{H}), 3.92(\mathrm{dd}, J=8.6,5.4,2 \mathrm{H}), 3.71(\mathrm{~m}, 1 \mathrm{H}), 2.44(\mathrm{~m}, 2 \mathrm{H}), 2.15(\mathrm{~m}, 1 \mathrm{H}), 1.88$ $(\mathrm{m}, 3 \mathrm{H}), 1.74(\mathrm{~s}, 1 \mathrm{H}), 1.26(\mathrm{t}, J=7.1,3 \mathrm{H})$; LRMS $(\mathrm{GC}-\mathrm{MS}, \mathrm{CI}) \mathrm{m} / \mathrm{z}$ calcd for $\mathrm{C}_{9} \mathrm{H}_{17} \mathrm{O}_{4}(\mathrm{M}+\mathrm{H})^{+} 189$, found 189.

C. The stereochemistry of alkene $\mathbf{1 3}$ was determined by conversion to $\mathbf{S 3 7}$ and subsequent analysis of the structure. Formation of the bicyclic compound indicates a 1,3-cis configuration, as a substrate with a 1,3 -trans configuration would not cyclize. ${ }^{28}$

Synthetic Scheme.

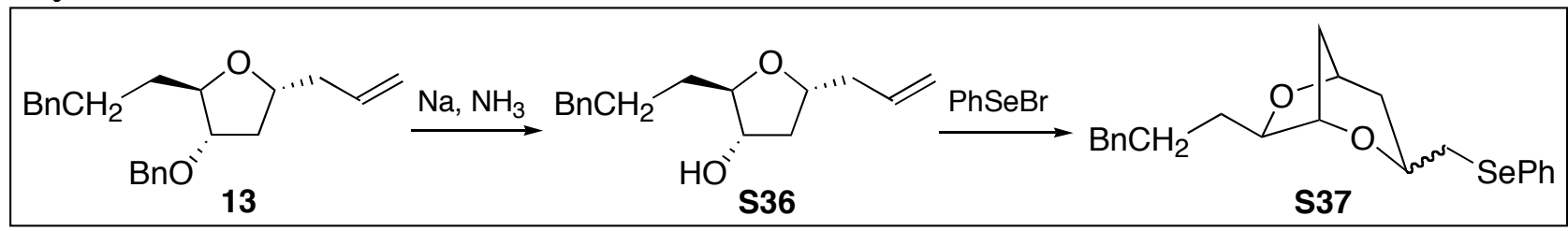


General procedure for benzyl group removal with sodium and ammonia: A cooled $\left(-78^{\circ} \mathrm{C}\right) 2$-necked, 10 $\mathrm{mL}$ flask was fitted with a cold finger $\left(-78^{\circ} \mathrm{C}\right)$ and treated with a stream of $\mathrm{NH}_{3}$ gas until approximately $5 \mathrm{~mL}$ of liquid had collected. Sodium metal (4 equiv) was added and the mixture was stirred for 10 min or until a deep blue color persisted. The substrate, dissolved in $2 \mathrm{~mL}$ of THF was added and if the blue color faded, an additional 4 equiv of sodium was added. After $10 \mathrm{~min}, \mathrm{MeOH}(2-5 \mathrm{~mL})$ was added and the mixture was maintained at $-78{ }^{\circ} \mathrm{C}$ for $30 \mathrm{~min}$ or until all of the sodium had disappeared. After $30 \mathrm{~min}$ at $22{ }^{\circ} \mathrm{C}$, the mixture was partitioned between saturated aqueous $\mathrm{NaH}_{2} \mathrm{PO}_{4}$ and $\mathrm{CH}_{2} \mathrm{Cl}_{2}$. The organic layer was saved and the aqueous layer was extracted with $2 \square 10 \mathrm{~mL}$ of $\mathrm{CH}_{2} \mathrm{Cl}_{2}$. Concentration in vacuo and flash chromatography afforded the product as a colorless oil.

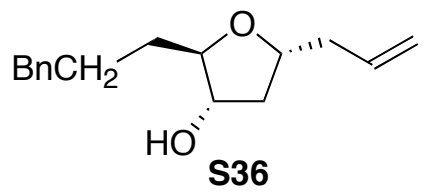

$\left(1 R^{*}, 3 S^{*}, \mathbf{4} R^{*}\right)$-1-Allyl-3-hydroxy-4-(3-phenylpropyl)tetrahydrofuran (S36). The standard metal reduction procedure was followed with alkene $13(0.311 \mathrm{~g}, 0.924 \mathrm{mmol})$ and flash chromatography (10:90 EtOAc/hexanes) to yield the product as a colorless oil $(0.184 \mathrm{~g}, 82 \%):{ }^{1} \mathrm{H} \mathrm{NMR}\left(500 \mathrm{MHz}, \mathrm{CDCl}_{3}\right) \square 7.26(\mathrm{~m}$, 2H), $7.16(\mathrm{~m}, 3 \mathrm{H}), 5.81(\mathrm{~m}, 1 \mathrm{H}), 5.09(\mathrm{~m}, 1 \mathrm{H}), 4.02(\mathrm{~m}, 2 \mathrm{H}), 3.79(\mathrm{~m}, 1 \mathrm{H}), 2.64(\mathrm{t}, J=7.7,2 \mathrm{H}), 2.42(\mathrm{~m}, 1 \mathrm{H})$, $2.31(\mathrm{~m}, 2 \mathrm{H}), 1.82(\mathrm{~m}, 2 \mathrm{H}), 1.69(\mathrm{~m}, 2 \mathrm{H}), 1.50(\mathrm{~m}, 2 \mathrm{H}) ;{ }^{13} \mathrm{C} \mathrm{NMR}\left(125 \mathrm{MHz}, \mathrm{CDCl}_{3}\right) \square 142.2,134.6,128.4$, 128.2, 125.7, 117.3, 84.9, 76.4, 76.3, 40.6, 39.8, 35.8, 32.4, 27.6; IR (thin film) 3415, 2935, 2860, 1086,916 $\mathrm{cm}^{-1}$; HRMS (CI/isobutane) $\mathrm{m} / \mathrm{z}$ calcd for $\mathrm{C}_{16} \mathrm{H}_{23} \mathrm{O}_{2}(\mathrm{M}+\mathrm{H})^{+} 247.1698$, found 247.1698.

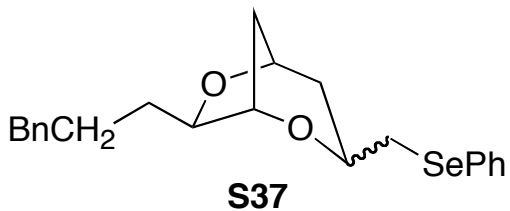

Bicyclic tetrahydropyran (S37). To a cooled $\left(-78^{\circ} \mathrm{C}\right)$ solution of $\mathbf{S 3 6}(0.072 \mathrm{~g}, 0.29 \mathrm{mmol})$ in $10 \mathrm{~mL}$ of $\mathrm{CH}_{2} \mathrm{Cl}_{2}$ and $\mathrm{K}_{2} \mathrm{CO}_{3}(0.460 \mathrm{~g})$ was added phenylselenenyl bromide $(0.655 \mathrm{~g}, 0.278 \mathrm{mmol})$. The resultant purple reaction mixture was capped with a polyethylene stopper and sealed with electrical tape. The reaction mixture was warmed to $22{ }^{\circ} \mathrm{C}$ over $12 \mathrm{~h}$, during which time the purple color had faded to bright yellow. The mixture was filtered through cotton and concentrated in vacuo. Purification by flash chromatography (5:95 - 20:80 EtOAc/hexanes) provided the product $\mathbf{S 3 7}$ as an oily white solid $(0.061 \mathrm{~g}, 52 \%)$, and the starting material $\mathbf{S 3 6}$ as a colorless oil $(0.026 \mathrm{~g}, 36 \%)$. Analysis of the product by HPLC $\left(\mathrm{SiO}_{2}, 0.5: 99.5 \mathrm{i}\right.$-PrOH/hexanes) indicated that the product was formed as a 92:8 mixture of diastereomers. The two diastereomers were separable in small amounts by preparative HPLC ( $\mathrm{SiO}_{2}, 0.5: 99.5 i$-PrOH/hexanes). HRMS and IR data were obtained for $\mathbf{S 3 7}$ as a mixture of diastereomers: IR (thin film) 2940, 1946, 1873, 1579, 1478, $1299 \mathrm{~cm}^{-1}$; HRMS (CI/isobutane) $\mathrm{m} / z$ calcd for $\mathrm{C}_{22} \mathrm{H}_{26} \mathrm{O}_{2} \mathrm{Se}(\mathrm{M})^{+} 402.1098$, found 402.1100 .

Major isomer of S37: ${ }^{1} \mathrm{H}$ NMR $\left(500 \mathrm{MHz}, \mathrm{CDCl}_{3}\right) \square 7.50(\mathrm{~m}, 2 \mathrm{H}), 7.28-7.20(\mathrm{~m}, 5 \mathrm{H}), 7.18(\mathrm{~m}, 3 \mathrm{H}), 4.50(\mathrm{~m}$, $1 \mathrm{H}), 4.17(\mathrm{~m}, 3 \mathrm{H}), 3.02(\mathrm{~m}, 2 \mathrm{H}), 2.63(\mathrm{t}, J=7.6,2 \mathrm{H}), 1.90(\mathrm{~m}, 1 \mathrm{H}), 1.77(\mathrm{~m}, 3 \mathrm{H}), 1.64(\mathrm{~m}, 1 \mathrm{H}), 1.36(\mathrm{~m}, 3 \mathrm{H})$; ${ }^{13} \mathrm{C}$ NMR $\left(125 \mathrm{MHz}, \mathrm{CDCl}_{3}\right) \square 142.0,132.6,130.5,129.0,128.4,128.3,126.9,125.8,81.4,78.9,74.1,69.7$, $38.9,35.8,35.6,34.2,33.8,27.6$.

Minor isomer of S37: ${ }^{1} \mathrm{H} \mathrm{NMR}\left(500 \mathrm{MHz}, \mathrm{CDCl}_{3}\right) \square 7.50(\mathrm{~m}, 2 \mathrm{H}), 7.30-7.21(\mathrm{~m}, 5 \mathrm{H}), 7.18(\mathrm{~m}, 3 \mathrm{H}), 4.47(\mathrm{t}, J$ $=5.5,1 \mathrm{H}), 4.16(\mathrm{~d}, J=1.8,1 \mathrm{H}), 3.93(\mathrm{~m}, 2 \mathrm{H}), 3.99(\mathrm{dd}, J=12.0,6.2,1 \mathrm{H}), 2.98(\mathrm{dd}, J=12.1,6.4,1 \mathrm{H}), 2.63(\mathrm{t}$, $J=7.6,2 \mathrm{H}), 2.09(\mathrm{~m}, 2 \mathrm{H}), 1.78(\mathrm{~m}, 2 \mathrm{H}), 1.64(\mathrm{~m}, 2 \mathrm{H}), 1.32(\mathrm{~m}, 2 \mathrm{H}) ;{ }^{13} \mathrm{C} \mathrm{NMR}\left(125 \mathrm{MHz}, \mathrm{CDCl}_{3}\right) \square 142.1$, $132.5,130.4,129.1,128.4,128.3,126.8,125.7,84.2,77.3,72.8,66.8,39.3,35.7,34.8,33.5,29.7,27.7 ;{ }^{13} \mathrm{C}$ 
NMR (125 MHz, $\left.\mathrm{CD}_{2} \mathrm{Cl}_{2}\right) \square 142.8,132.6,131.2,129.4,128.8,128.6,127.0,126.0,85.2,77.6,73.1,67.1,39.8$, $36.0,35.2,34.0,30.1,28.2$.

D. The stereochemistry of alkene $\mathbf{1 7}$ was determined by analysis of nOe data:

Relevant DPFGSE-nOe data (mixing time $0.5 \mathrm{~s}$ ): (the peaks in the ${ }^{1} \mathrm{H}$ NMR spectra were assigned using ${ }^{1} \mathrm{H} /{ }^{1} \mathrm{H}$ COSY, ${ }^{1} \mathrm{H}$ NMR chemical shifts, and ${ }^{1} \mathrm{H}$ NMR coupling constants)

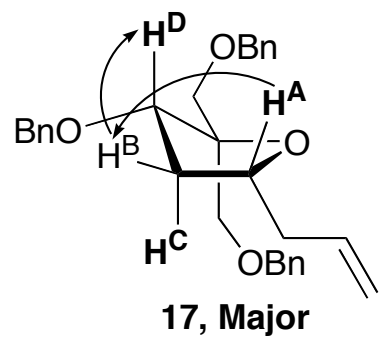

\section{1-Allyl-3,5',5-tri-O-benzyl-2-deoxy-4-(hydroxymethyl)ribose (1,3-cis, major)}

nOe experiments were performed for major $\mathbf{1 7}$ using a pure sample of the isomer $\mathbf{H}^{\mathrm{A}}$ irradiated: $\mathrm{H}^{\mathrm{B}}(3.2 \%)$

$\mathbf{H}^{\mathrm{C}}$ irradiated: $\mathrm{H}^{\mathrm{A}}(0.8 \%), \mathrm{H}^{\mathrm{D}}(0.7 \%)$

$\mathbf{H}^{\mathrm{D}}$ irradiated: $\mathrm{H}^{\mathrm{C}}(0.7 \%), \mathrm{H}^{\mathrm{B}}(2.5 \%)$

Note: The observation of large nOe enhancements between $\mathrm{H}^{\mathrm{A}}$ and $\mathrm{H}^{\mathrm{B}}$ and between $\mathrm{H}^{\mathrm{B}}$ and $\mathrm{H}^{\mathrm{D}}$ suggests a 1,3 -cis configuration. ${ }^{29}$

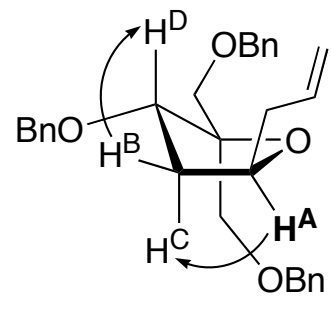

17, minor

1-Allyl-3,5',5-tri- $O$-benzyl-2-deoxy-4-(hydroxymethyl)ribose (1,3-trans, minor) nOe experiments were performed for minor $\mathbf{1 7}$ using a pure sample of the isomer $\mathbf{H}^{\mathbf{A}}$ irradiated: $\mathrm{H}^{\mathrm{C}}(2.7 \%)$

$\mathbf{H}^{\mathrm{B}}$ irradiated: $\mathrm{H}^{\mathrm{D}}(3.4 \%), \mathrm{H}^{\mathrm{A}}(0.6 \%)$

$\mathbf{H}^{\mathrm{C}}$ irradiated: $\mathrm{H}^{\mathrm{A}}(3.1 \%), \mathrm{H}^{\mathrm{D}}(1.3 \%)$

$\mathbf{H}^{\mathrm{D}}$ irradiated: $\mathrm{H}^{\mathrm{C}}(0.9 \%), \mathrm{H}^{\mathrm{B}}(2.5 \%)$

Note: The observation of large nOe enhancements between $\mathrm{H}^{\mathrm{A}}$ and $\mathrm{H}^{\mathrm{C}}$ and between $\mathrm{H}^{\mathrm{B}}$ and $\mathrm{H}^{\mathrm{D}}$ suggests a 1,3-trans configuration.

E. The stereochemistry of alkene $\mathbf{4 1}$ was assigned by conversion of $\mathbf{4 1}$ to $\mathbf{S 3 8}$, whose relative stereochemistry was determined by analysis of nOe data.

Synthetic Scheme. 


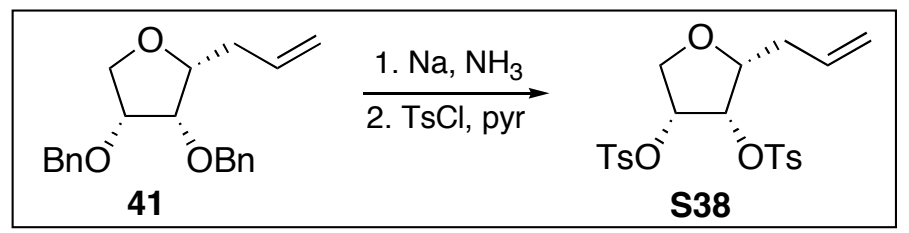

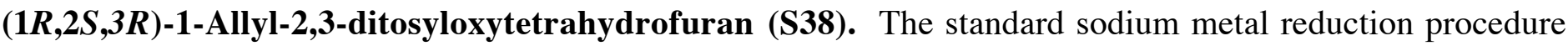
was followed with alkene $41(0.325 \mathrm{~g}, 1.00 \mathrm{mmol})$. Because the resultant diol was soluble in $\mathrm{H}_{2} \mathrm{O}$, the mixture was absorbed to silica gel following treatment with $\mathrm{MeOH}$. Purification by flash chromatography (80:20:0 90:0:10 EtOAc/hexanes/MeOH) provided the product as a colorless oil $(0.0263 \mathrm{~g}, 18 \%)$, which was converted to the tosylate for characterization. To a cooled $\left(0{ }^{\circ} \mathrm{C}\right)$ solution of diol $(0.0263 \mathrm{~g}, 0.182 \mathrm{mmol})$ in $2 \mathrm{~mL}$ of pyridine (dried over $4 \AA$ molecular sieves) was added $\mathrm{TsCl}(0.348 \mathrm{~g}, 1.82 \mathrm{mmol})$. After the reaction mixture was stirred for $24 \mathrm{~h}$ at $22{ }^{\circ} \mathrm{C}$, the solution was filtered through Celite and concentrated in vacuo. The resultant residue was dissolved in $2 \mathrm{M} \mathrm{HCl}$ and extracted with $3 \square 10 \mathrm{~mL}$ of $\mathrm{CH}_{2} \mathrm{Cl}_{2}$. The combined organic phases were washed with $10 \mathrm{~mL}$ of $2 \mathrm{M} \mathrm{HCl}$ and $10 \mathrm{~mL}$ of saturated aqueous $\mathrm{NaHCO}_{3}$, dried $\left(\mathrm{Na}_{2} \mathrm{SO}_{4}\right)$, and concentrated in vасио. Purification by flash chromatography (2:98 - 20:80 EtOAc/hexanes) provided the product as a colorless oil $(0.076 \mathrm{~g}, 92 \%)$.

Major isomer of S38: ${ }^{1} \mathrm{H}$ NMR $\left(500 \mathrm{MHz}, \mathrm{CDCl}_{3}\right) \square 7.80(\mathrm{~d}, J=8.3,2 \mathrm{H}), 7.72(\mathrm{~d}, J=8.3,2 \mathrm{H}), 7.35(\mathrm{~m}, 4 \mathrm{H})$, $5.74(\mathrm{ddt}, J=17.2,10.3,6.9,1 \mathrm{H}), 5.08(\mathrm{~m}, 2 \mathrm{H}), 5.02(\mathrm{t}, J=4.7,1 \mathrm{H}), 4.91(\mathrm{td}, J=6.6,5.1,1 \mathrm{H}), 3.96(\mathrm{~m}, 1 \mathrm{H})$, $3.89(\mathrm{dd}, J=9.8,6.7,1 \mathrm{H}), 3.82(\mathrm{dd}, J=9.8,6.3,1 \mathrm{H}), 2.47(\mathrm{~s}, 6 \mathrm{H}), 2.36(\mathrm{~m}, 2 \mathrm{H}) ;{ }^{13} \mathrm{C} \mathrm{NMR}\left(125 \mathrm{MHz} \mathrm{CDCl}_{3}\right)$ $\square$ 145.6, 145.4, 133.6, 133.4, 132.9, 130.2, 130.1, 128.28, 128.25, 118.0, 78.7, 78.0, 76.2, 68.5, 34.2, 21.9; IR (thin film) 3069, 2923, 1597, 1373, $1190 \mathrm{~cm}^{-1}$; HRMS (EI) $\mathrm{m} / \mathrm{z}$ calcd for $\mathrm{C}_{21} \mathrm{H}_{24} \mathrm{O}_{7} \mathrm{~S}_{2} \mathrm{Na}(\mathrm{M}+\mathrm{Na})^{+} 475.0861$, found 475.0864. Anal. Calcd for $\mathrm{C}_{21} \mathrm{H}_{24} \mathrm{O}_{7} \mathrm{~S}_{2}$ : C, 55.74; H, 5.35. Found: C, 55.45; H, 5.43.

Minor isomer of S38: ${ }^{1} \mathrm{H}$ NMR $\left(500 \mathrm{MHz}, \mathrm{C}_{6} \mathrm{D}_{6}\right) \square 7.78(\mathrm{~m}, 4 \mathrm{H}), 6.71(\mathrm{~m}, 4 \mathrm{H}), 5.60(\mathrm{~m}, 1 \mathrm{H}), 4.90(\mathrm{~m}, 3 \mathrm{H})$, $4.54(\mathrm{dd}, J=7.0,5.5,1 \mathrm{H}), 4.00(\mathrm{td}, J=6.7,4.6,1 \mathrm{H}), 3.85(\mathrm{dd}, J=10.7,3.9,1 \mathrm{H}), 3.60(\mathrm{dd}, J=10.7,5.0,1 \mathrm{H})$, $2.17(\mathrm{~m}, 1 \mathrm{H}), 1.95(\mathrm{~m}, 1 \mathrm{H}), 1.81(\mathrm{~s}, 6 \mathrm{H})$.

Relevant DPFGSE-nOe data (mixing time 2.0 s): (the peaks in the ${ }^{1} \mathrm{H}$ NMR spectra were assigned using ${ }^{1} \mathrm{H} /{ }^{1} \mathrm{H}$ COSY, ${ }^{1} \mathrm{H}$ NMR chemical shifts, and ${ }^{1} \mathrm{H}$ NMR coupling constants)

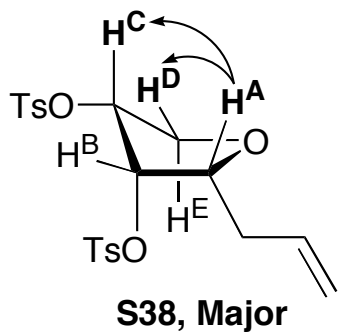

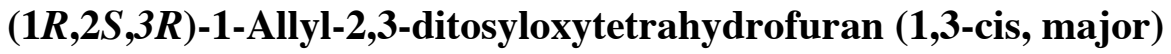

nOe experiments were performed for 1,3-cis $\mathbf{S 3 8}$ using a pure sample of the isomer in $\mathrm{C}_{6} \mathrm{D}_{6}$

$\mathbf{H}^{\mathrm{A}}$ irradiated: $\mathrm{H}^{\mathrm{D}}(1.0 \%), \mathrm{H}^{\mathrm{C}}(4.1 \%)$

H $^{\mathrm{C}}$ irradiated: $\mathrm{H}^{\mathrm{A}}(4.4 \%)$

$\mathbf{H}^{\mathrm{D}}$ irradiated: $\mathrm{H}^{\mathrm{A}}(1.9 \%)$

Note: The observation of nOe between $\mathrm{H}^{\mathrm{A}}$ and $\mathrm{H}^{\mathrm{C}}$ along with $\mathrm{H}^{\mathrm{D}}$ suggests a 1,3-cis configuration. 


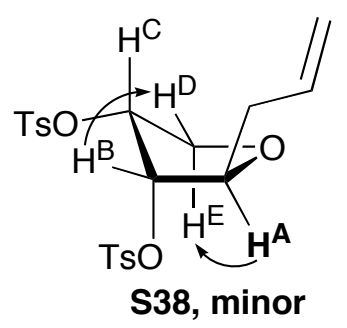

(1S,2S,3R)-1-Allyl-2,3-ditosyloxytetrahydrofuran (1,3-trans, minor)

nOe experiments were performed for 1,3-trans $\mathbf{S 3 8}$ using a pure sample of the isomer in $\mathrm{C}_{6} \mathrm{D}_{6}$

$\mathbf{H}^{\mathrm{A}}$ irradiated: $\mathrm{H}^{\mathrm{E}}(1.4 \%)$

$\mathbf{H}^{\mathbf{B}}$ irradiated: $\mathrm{H}^{\mathrm{D}}(1.3 \%)$

$\mathbf{H}^{\mathrm{D}}$ irradiated: $\mathrm{H}^{\mathrm{B}}(1.5 \%)$

$\mathbf{H}^{\mathrm{E}}$ irradiated: $\mathrm{H}^{\mathrm{A}}(1.8 \%)$

Note: The observation of nOe between $\mathrm{H}^{\mathrm{A}}$ and $\mathrm{H}^{\mathrm{E}}$ and the absence of nOe between $\mathrm{H}^{\mathrm{A}}$ and $\mathrm{H}^{\mathrm{C}}$ suggests a 1,3-trans configuration.

F. The stereochemistry of alkene $\mathbf{4 3}$ was assigned by conversion of $\mathbf{4 3}$ to $\mathbf{S 3 9}$, whose relative stereochemistry was determined by analysis of nOe data.

Synthetic Scheme.

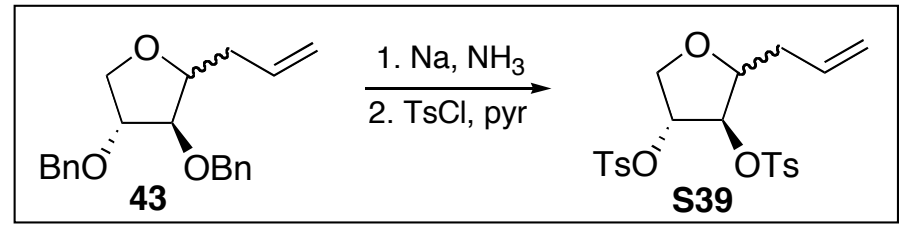

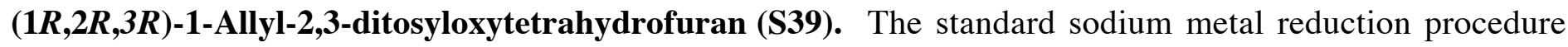
was followed with alkene $43(1.06 \mathrm{~g}, 3.27 \mathrm{mmol})$. Because the resultant diol was soluble in water, the mixture was absorbed to silica gel following treatment with $\mathrm{MeOH}$. Purification by flash chromatography (20:80 80:20 EtOAc/hexanes) provided the product as a colorless oil $(0.270 \mathrm{~g}, 57 \%)$, which was converted to the tosylate for characterization. To a cooled $\left(0^{\circ} \mathrm{C}\right)$ solution of diol $(0.100 \mathrm{~g}, 0.694 \mathrm{mmol})$ in $5 \mathrm{~mL}$ of pyridine (dried over $4 \AA$ molecular sieves) was added TsCl (1.32 g, $6.94 \mathrm{mmol})$. After the reaction mixture was stirred for $24 \mathrm{~h}$ at $22^{\circ} \mathrm{C}$, the solution was filtered through Celite and concentrated in vacuo. The resultant residue was dissolved in $2 \mathrm{M}$ aqueous $\mathrm{HCl}$ and extracted with $3 \square 10 \mathrm{~mL}$ of $\mathrm{CH}_{2} \mathrm{Cl}_{2}$. The combined organic phases were washed with $10 \mathrm{~mL}$ of $2 \mathrm{M}$ aqueous $\mathrm{HCl}$ and $10 \mathrm{~mL}$ of saturated aqueous $\mathrm{NaHCO}_{3}$, dried $\left(\mathrm{Na}_{2} \mathrm{SO}_{4}\right)$, and concentrated in vacuo. Purification by flash chromatography $(5: 95-10: 90 \mathrm{EtOAc/hexanes)}$ provided the product as a colorless oil $(0.283 \mathrm{~g}, 90 \%)$. The purified product was characterized as a mixture of diastereomers: ${ }^{1} \mathrm{H}$ NMR $\left(500 \mathrm{MHz} \mathrm{CDCl}_{3}\right) \square 7.74-7.80(\mathrm{~m}, 11.2 \mathrm{H}), 7.37(\mathrm{~m}, 11.2 \mathrm{H}), 5.55(\mathrm{~m}, 2.8 \mathrm{H}), 4.93-5.03(\mathrm{~m}, 8.4 \mathrm{H})$, $4.85(\mathrm{~d}, J=3.0,1 \mathrm{H}), 4.64(\mathrm{~d}, J=3.3,1.8 \mathrm{H}), 4.11(\mathrm{dd}, J=11.1,4.7,1 \mathrm{H}), 4.02(\mathrm{ddd}, J=8.4,5.9,3.1,1 \mathrm{H}), 3.96$ $(\mathrm{d}, J=11.3,1.8 \mathrm{H}), 3.85(\mathrm{~m}, 3.6 \mathrm{H}), 3.73(\mathrm{dd}, J=11.2,1.5,1 \mathrm{H}), 2.47(\mathrm{~s}, 10.8 \mathrm{H}), 2.46(\mathrm{~s}, 6 \mathrm{H}), 2.34(\mathrm{~m}, 1 \mathrm{H}), 2.27$ $(\mathrm{m}, 3.6 \mathrm{H}), 2.16(\mathrm{~m}, 1 \mathrm{H}) ;{ }^{13} \mathrm{C}$ NMR $\left(125 \mathrm{MHz} \mathrm{CDCl}_{3}\right) \square 145.8,145.7,133.2,133.0,132.7,132.6,130.31$, $130.29,130.27,130.25,128.42,128.40,128.35,128.3,118.5,118.0,84.9,83.2,82.8,82.3,82.1,79.2,71.2$, 71.0, 36.7, 32.8, 21.9; IR (thin film) 3070, 2980, 1643, 1597, 1373, $1190 \mathrm{~cm}^{-1}$; HRMS (EI) $\mathrm{m} / \mathrm{z}$ calcd for 
$\mathrm{C}_{21} \mathrm{H}_{24} \mathrm{O}_{7} \mathrm{~S}_{2} \mathrm{Na}(\mathrm{M}+\mathrm{Na})^{+}$475.0861, found 475.0866. Anal. Calcd for $\mathrm{C}_{21} \mathrm{H}_{24} \mathrm{O}_{7} \mathrm{~S}_{2}: \mathrm{C}, 55.74 ; \mathrm{H}, 5.35$. Found: $\mathrm{C}$, 55.74; H, 5.09 .

Relevant DPFGSE-nOe data (mixing time $2.0 \mathrm{~s}$ ): (the peaks in the ${ }^{1} \mathrm{H}$ NMR spectra were assigned using ${ }^{1} \mathrm{H} /{ }^{1} \mathrm{H}$ COSY, ${ }^{1} \mathrm{H}$ NMR chemical shifts, and ${ }^{1} \mathrm{H}$ NMR coupling constants)

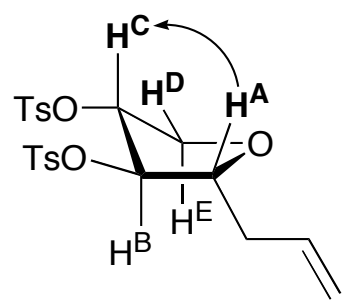

S39, major

(1R,2R,3R)-1-Allyl-2,3-ditosyloxytetrahydrofuran (1,3-cis, major)

nOe experiments were performed for 1,3-cis $\mathbf{S 3 9}$ using a 64:36 mixture of cis:trans isomers in $\mathrm{CD}_{3} \mathrm{OD}$

$\mathbf{H}^{\mathrm{A}}$ irradiated: $\mathrm{H}^{\mathrm{C}}(0.9 \%)$

$\mathbf{H}^{\mathrm{B}}$ irradiated: $\mathrm{H}^{\mathrm{A}}(2.5 \%), \mathrm{H}^{\mathrm{C}}(3.6 \%)$

Note: The observation of nOe between $\mathrm{H}^{\mathrm{A}}$ and $\mathrm{H}^{\mathrm{C}}$ along with similar nOe values for $\mathrm{H}^{\mathrm{B}} \square \mathrm{H}^{\mathrm{C}}$ and $\mathrm{H}^{\mathrm{B}} \square \mathrm{H}^{\mathrm{A}}$ suggests a 1,3-cis configuration.

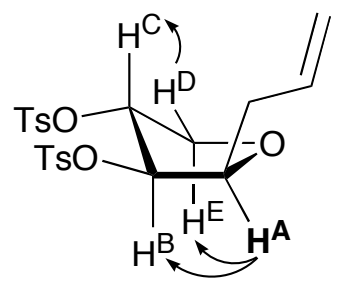

S39, minor

(1S,2R,3R)-1-Allyl-2,3-ditosyloxytetrahydrofuran (1,3-trans, minor)

nOe experiments were performed for 1,3-trans $\mathbf{S 3 9}$ using a 64:36 mixture of cis:trans isomers in $\mathrm{CD}_{3} \mathrm{OD}$

$\mathbf{H}^{\mathbf{A}}$ irradiated: $\mathrm{H}^{\mathrm{E}}(1.7 \%), \mathrm{H}^{\mathrm{B}}(9.7 \%)$

$\mathbf{H}^{\mathrm{D}}$ irradiated: $\mathrm{H}^{\mathrm{C}}(10.1 \%)$

$\mathbf{H}^{\mathrm{E}}$ irradiated: $\mathrm{H}^{\mathrm{A}}(2.6 \%), \mathrm{H}^{\mathrm{C}}(2.6 \%)$

Note: The observation of nOe between $\mathrm{H}^{\mathrm{A}}$ and $\mathrm{H}^{\mathrm{E}}$ along with a large nOe enhancement to $\mathrm{H}^{\mathrm{B}}$ suggests a 1,3-trans configuration.

G. The stereochemistry of alkene $\mathbf{5 1}$ was determined by conversion to $\mathbf{S 4 1}$ and subsequent NMR analysis of the structure (COSY, HMQC, HMBC, and TOCSY). The 1,3-cis configuration promotes cyclization to afford $\mathbf{S} 41$.

Synthetic Scheme. 


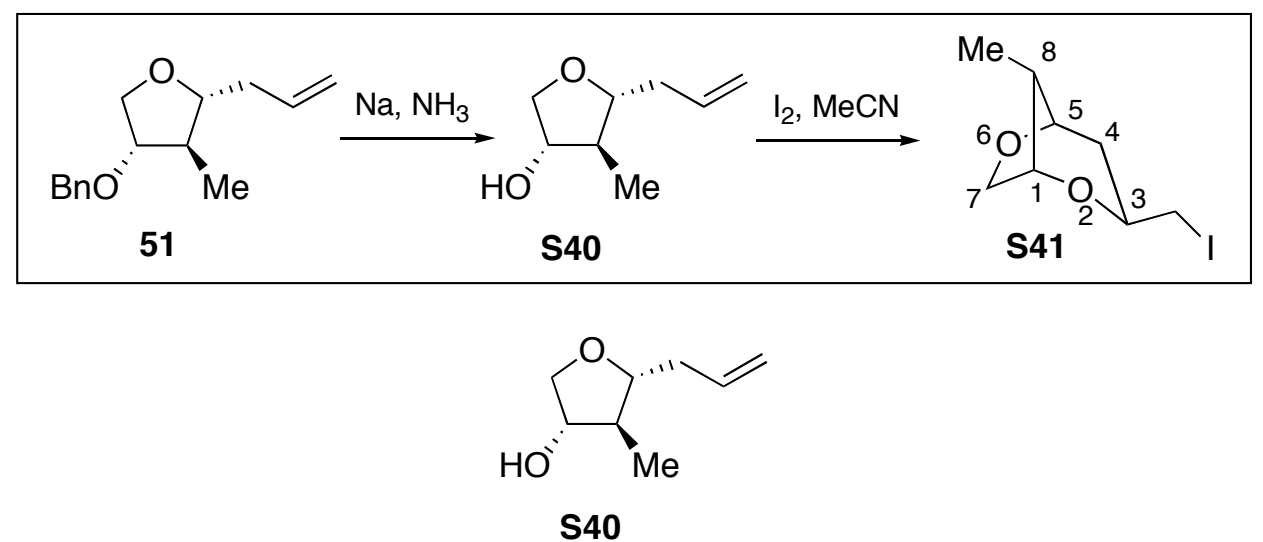

$\left(1 R^{*}, 2 R^{*}, 3 S^{*}\right)$-1-Allyl-3-hydroxy-2-methyltetrahydrofuran ( $\left.\mathbf{S 4 0}\right)$. The standard sodium metal reduction procedure was followed with alkene $51(0.07 \mathrm{~g}, 0.3 \mathrm{mmol})$. Purification by flash chromatography (10:9030:70 EtOAc/hexanes) provided the product as an oil $(0.03 \mathrm{~g}, 70 \%):{ }^{1} \mathrm{H}$ NMR $\left(500 \mathrm{MHz}, \mathrm{CDCl}_{3}\right) \square 5.86$ (ddt, $J$ $=17.2,10.2,7.0,1 \mathrm{H}), 5.14(\mathrm{ddd}, J=17.2,3.4,1.5,1 \mathrm{H}), 5.10(\mathrm{ddt}, J=10.2,2.1,1.1,1 \mathrm{H}), 3.96(\mathrm{~m}, 1 \mathrm{H}), 3.83$ (dd, $J=9.7,5.1,1 \mathrm{H}), 3.74(\mathrm{dd}, J=9.7,3.3,1 \mathrm{H}), 3.45(\mathrm{td}, J=7.1,5.2,1 \mathrm{H}), 2.39(\mathrm{~m}, 2 \mathrm{H}), 2.12(\mathrm{~s}, 1 \mathrm{H}), 1.82(\mathrm{~m}$, $1 \mathrm{H}), 1.06(\mathrm{~d}, J=7.0,3 \mathrm{H}) ;{ }^{13} \mathrm{C}$ NMR $\left(125 \mathrm{MHz}, \mathrm{CDCl}_{3}\right) \square 134.5,117.3,85.1,79.3,73.7,47.5,38.8,15.9$; IR (thin film) $3398,3077,2928,2873,1642 \mathrm{~cm}^{-1}$; HRMS (GC-MS, EI) $\mathrm{m} / z$ calcd for $\mathrm{C}_{5} \mathrm{H}_{9} \mathrm{O}_{2}\left(\mathrm{M}-\mathrm{C}_{3} \mathrm{H}_{5}\right)^{+}$ 101.0602, found 101.0605.

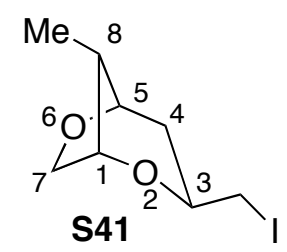

$\left(1 S^{*}, 5 R^{*}, 8 S^{*}\right)$-3-Iodomethyl-8-methyl-2,6-dioxabicyclo[3.2.1]octane (S41). To a cooled $\left(0{ }^{\circ} \mathrm{C}\right)$ solution of alcohol $\mathbf{S 4 0}(0.036 \mathrm{~g}, 0.25 \mathrm{mmol})$ in $\mathrm{MeCN}(15 \mathrm{~mL})$ was added $\mathrm{I}_{2}(0.200 \mathrm{~g}, 0.788 \mathrm{mmol})$. The flask was protected from light and the solution was stirred for $12 \mathrm{~h}$. Saturated aqueous $\mathrm{Na}_{2} \mathrm{~S}_{2} \mathrm{O}_{3}(10 \mathrm{~mL})$ was added and the mixture was vigorously stirred until colorless. $\mathrm{Et}_{2} \mathrm{O}(30 \mathrm{~mL})$ was added and the layers were separated. The aqueous layer was extracted with $2 \square 10 \mathrm{~mL}$ of $\mathrm{Et}_{2} \mathrm{O}$. The combined organic layers were dried $\left(\mathrm{Na}_{2} \mathrm{SO}_{4}\right)$ and concentrated in vacuo. ${ }^{1} \mathrm{H}$ NMR spectroscopic analysis of the unpurified product showed a single diastereomer. Purification by flash chromatography (10:90 - 30:70 EtOAc/hexanes) provided the product as a colorless oil $(0.051 \mathrm{~g}, 75 \%):{ }^{1} \mathrm{H}$ NMR $\left(500 \mathrm{MHz}, \mathrm{CDCl}_{3}\right) \square 4.21(\mathrm{~d}, J=2.7,1 \mathrm{H}), 4.18(\mathrm{~d}, J=4.7,1 \mathrm{H}), 4.08(\mathrm{~d}, J=10.4$, $1 \mathrm{H}), 3.92(\mathrm{dd}, J=10.4,3.1,1 \mathrm{H}), 3.79(\mathrm{~m}, 1 \mathrm{H}), 3.30(\mathrm{dd}, J=10.3,4.6,1 \mathrm{H}), 3.19(\mathrm{dd}, J=10.3,5.3,1 \mathrm{H}), 2.04$ $(\mathrm{m}, 1 \mathrm{H}), 1.97(\mathrm{dt}, J=12.9,4.6,1 \mathrm{H}), 1.44(\mathrm{~m}, 1 \mathrm{H}), 0.90(\mathrm{~d}, J=5.0,3 \mathrm{H}) ;{ }^{13} \mathrm{C}$ NMR $\left(125 \mathrm{MHz}, \mathrm{CDCl}_{3}\right) \square 80.4$, 78.9, 69.4, 68.4, 43.3, 39.6, 14.3, 10.9; IR (thin film) 2960, 2881, $1071 \mathrm{~cm}^{-1}$; HRMS (GC-MS, EI) $\mathrm{m} / \mathrm{z}$ calcd for $\mathrm{C}_{8} \mathrm{H}_{13} \mathrm{O}_{2} \mathrm{I}(\mathrm{M})^{+} 267.9962$, found 267.9960 .

H. The stereochemistry of alkene $\mathbf{5 3}$ was determined by conversion to $\mathbf{S 4 3}$ and NMR analysis of the structure (COSY, HMQC, HMBC, and TOCSY). The 1,3-cis configuration promotes cyclization to afford $\mathbf{S 4 3}$.

Synthetic Scheme. 


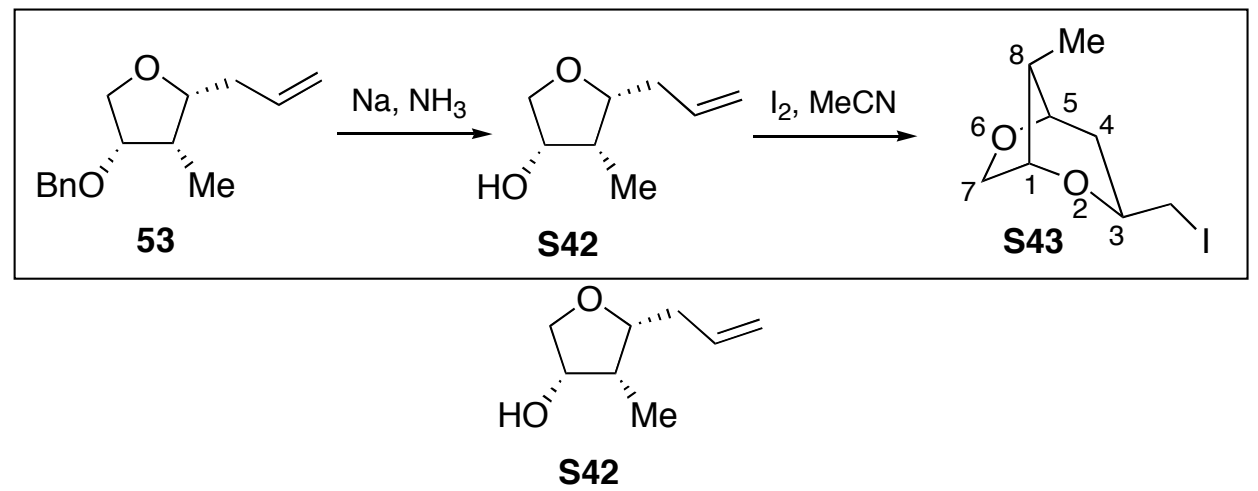

$\left(1 R^{*}, 2 S *, 3 S^{*}\right)$-1-Allyl-3-hydroxy-2-methyltetrahydrofuran (S42). The standard sodium metal reduction procedure was followed with alkene $53(0.115 \mathrm{~g}, 0.496 \mathrm{mmol})$. Purification by flash chromatography (20:80 EtOAc/hexanes) provided the product as an oil $(0.031 \mathrm{~g}, 44 \%):{ }^{1} \mathrm{H} \mathrm{NMR}\left(500 \mathrm{MHz}, \mathrm{CDCl}_{3}\right) \square 5.86(\mathrm{ddt}, J=$ $17.2,10.2,6.8,1 \mathrm{H}), 5.12(\mathrm{ddd}, J=17.2,3.2,1.6,1 \mathrm{H}), 5.07(\mathrm{dd}, J=10.2,1.2,1 \mathrm{H}), 4.35(\mathrm{~m}, 1 \mathrm{H}), 3.99(\mathrm{ddd}, J=$ 9.0, 7.1, 4.7, 1H), $3.89(\mathrm{dd}, J=9.5,5.3,1 \mathrm{H}), 3.76(\mathrm{dd}, J=9.5,3.3,1 \mathrm{H}), 2.33(\mathrm{~m}, 2 \mathrm{H}), 2.23(\mathrm{~m}, 1 \mathrm{H}), 2.00(\mathrm{~d}, J$ $=4.6,1 \mathrm{H}), 1.02(\mathrm{~d}, J=7.3,3 \mathrm{H}) ;{ }^{13} \mathrm{C} \mathrm{NMR}\left(125 \mathrm{MHz}, \mathrm{CDCl}_{3}\right) \square$ 135.6, 116.5, 80.5, 74.1, 73.4, 40.3, 36.0, 7.9; IR (thin film) 3417, 3076, 2939, 2882, $1643 \mathrm{~cm}^{-1}$; HRMS (GC-MS, EI) $m / z$ calcd for $\mathrm{C}_{5} \mathrm{H}_{9} \mathrm{O}_{2}\left(\mathrm{M}-\mathrm{C}_{3} \mathrm{H}_{5}\right)^{+}$ 101.0602, found 101.0602 .

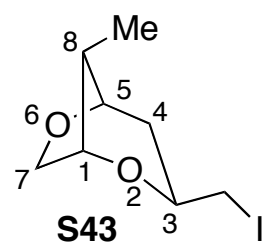

$\left(1 S^{*}, 5 R^{*}, 8 R^{*}\right)$-3-Iodomethyl-8-methyl-2,6-dioxabicyclo[3.2.1]octane $(\mathbf{S 4 3})$. To a cooled $\left(0{ }^{\circ} \mathrm{C}\right)$ solution of alcohol S42 $(0.031 \mathrm{~g}, 0.22 \mathrm{mmol})$ in $\mathrm{MeCN}(15 \mathrm{~mL})$ was added $\mathrm{I}_{2}(0.18 \mathrm{~g}, 0.70 \mathrm{mmol})$. The flask was protected from light and the solution was stirred for $12 \mathrm{~h}$. Saturated aqueous $\mathrm{Na}_{2} \mathrm{~S}_{2} \mathrm{O}_{3}(10 \mathrm{~mL})$ was added and the mixture was vigorously stirred until colorless. $\mathrm{Et}_{2} \mathrm{O}(30 \mathrm{~mL})$ was added and the layers were separated. The aqueous layer was extracted with $2 \square 10 \mathrm{~mL}$ of $\mathrm{Et}_{2} \mathrm{O}$. The combined organic layers were dried $\left(\mathrm{Na}_{2} \mathrm{SO}_{4}\right)$ and concentrated in vacuo. Purification by flash chromatography (10:90 - 30:70 EtOAc/hexanes) provided the product as a colorless oil $(0.051 \mathrm{~g}, 75 \%)$ and as a 75:25 ratio of separated diastereomers (determined by isolated yields after chromatography).

Major diastereomer of S43: ${ }^{1} \mathrm{H}$ NMR $\left(500 \mathrm{MHz} \mathrm{CDCl}_{3}\right) \square 4.29(\mathrm{t}, J=5.2,1 \mathrm{H}), 4.20(\mathrm{~s}, 1 \mathrm{H}), 4.19(\mathrm{~d}, J=13.8$, $1 \mathrm{H}), 3.91(\mathrm{~m}, 1 \mathrm{H}), 3.88(\mathrm{~m}, 1 \mathrm{H}), 3.31(\mathrm{dd}, J=10.4,4.8,1 \mathrm{H}), 3.25(\mathrm{dd}, J=10.4,5.6,1 \mathrm{H}), 2.00(\mathrm{~m}, 1 \mathrm{H}), 1.74$ $(\mathrm{dt}, J=13.4,4.6,1 \mathrm{H}), 1.60(\mathrm{dd}, J=13.2,10.7,1 \mathrm{H}), 1.23(\mathrm{~d}, J=6.9,3 \mathrm{H}) ;{ }^{13} \mathrm{C} \mathrm{NMR}\left(125 \mathrm{MHz}, \mathrm{CDCl}_{3}\right) \square 77.8$, 76.9, 71.3, 69.2 , 39.0, 33.3, 9.9, 9.4; IR (thin film) 2955, 2878, $1082 \mathrm{~cm}^{-1}$; HRMS (GC-MS, EI) $\mathrm{m} / \mathrm{z}$ calcd for $\mathrm{C}_{8} \mathrm{H}_{13} \mathrm{O}_{2} \mathrm{I}(\mathrm{M})^{+} 267.9962$, found 267.9950.

Minor diastereomer of S43: ${ }^{1} \mathrm{H}$ NMR $\left(500 \mathrm{MHz}, \mathrm{CDCl}_{3}\right) \square 4.33(\mathrm{~m}, 1 \mathrm{H}), 4.17(\mathrm{~m}, 2 \mathrm{H}), 3.93(\mathrm{~d}, J=10.2,1 \mathrm{H})$, $3.82(\mathrm{~m}, 1 \mathrm{H}), 3.44(\mathrm{~m}, 1 \mathrm{H}), 3.35(\mathrm{~m}, 1 \mathrm{H}), 1.95(\mathrm{~m}, 3 \mathrm{H}), 1.15(\mathrm{~d}, J=6.8,3 \mathrm{H}) ;{ }^{13} \mathrm{C} \mathrm{NMR}\left(125 \mathrm{MHz}, \mathrm{CDCl}_{3}\right) \square$ $76.7,72.9,70.3,36.9,29.6,14.8,9.8$.

\section{Bibliography}

1. Pangborn, A. B.; Giardello, M. A.; Grubbs, R. H.; Rosen, R. K.; Timmers, F. J. Organometallics 1996, 15, $1518-1520$.

2. Dahlman, O.; Garegg, P. J.; Mayer, H.; Schramek, S. Acta Chem. Scand. B. 1986, 40, 15-20. 
3. Stang, P. J.; Dueber, T. E. Organic Syntheses 1974, 54, 79-84.

4. Dahanukar, V. H.; Rychnovsky, S. D. J. Org. Chem. 1996, 61, 8317-8320.

5. Ishii, Y.; Osakada, K.; Ikariya, T.; Saburi, M.; Yoshikawa, S. J. Org. Chem. 1986, 51, 2034-2039.

6. Burke, S. D.; Ng, R. A.; Morrison, J. A.; Alberti, M. J. J. Org. Chem. 1998, 63, 3160-3161.

7. Burke, S. D.; Fobare, W. F.; Pacofsky, G. J. J. Org. Chem. 1983, 48, 5221-5228.

8. Basha, A.; Lipton, M.; Weinreb, S. M. Tetrahedron Lett. 1977, 18, 4171-4174.

9. Nahm, S.; Weinreb, S. M. Tetrahedron Lett. 1981, 22, 3815-3818.

10. Pikul, S.; Raczko, J.; Ankner, K.; Jurczak, J. J. Am. Chem. Soc. 1981, 109, 3981-3987.

11. Chong, J. M.; Mar, E. K. Tetrahedron 1989, 45, 7709-7716.

12. Castro, B. Bull. Soc. Chim. Fr. 1967, 1540-1547.

13. Nudelman, A.; Keiner, R.; Broida, N.; Gottlieb, H. G. Synthesis 1989, 387-388.

14. Danheiser, R. L.; Miller, R. F.; Brisbois, R. G.; Park, S. Z. J. Org. Chem. 1990, 55, 1959-1964.

15. Taber, D. F.; Ruckle, R. E., Jr.; Hennessy, M. J. J. Org. Chem. 1986, 51, 4077-4078.

16. Doyle, M. P.; Dyatkin, A. D.; Tedrow, J. S. Tetrahedron Lett. 1994, 35, 3853-3856.

17. Hanessian, S.; Murray, P. J. Tetrahedron 1987, 43, 5055-5072.

18. Aquino, M.; Cardini, S.; Fronza, G.; Fuganti, C. Tetrahedron 1991, 47, 7887-7896.

19. Dauban, P.; Chiaroni, A.; Riche, C.; Dodd, R. H. J. Org. Chem. 1996, 61, 2488-2496.

20. Marshall, J. A.; Seletsky, B. M.; Luke, G. P. J. Org. Chem. 1994, 59, 3413-3420.

21. Nemoto, H.; Takamatsu, S.; Yamamoto, Y. J. Org. Chem. 1991, 56, 1321-1322.

22. Fowler, P. A.; Haines, A. H.; Taylor, R. J. K.; Chrystal, E. J. T.; Gravestock, M. B. J. Chem. Soc., Perkin Trans. 1 1993, 1003-1005.

23. Chamberlin, A. R.; Dezube, M. Tetrahedron Lett. 1982, 23, 3055-3058.

24. Wessel, H. P.; Iversen, T.; Bundle, D. R. J. Chem. Soc., Perkin Trans. 1 1985, 2247-2250.

25. Shibata, I.; Matsuo, F.; Baba, A.; Matsuda, H. J. Org. Chem. 1991, 56, 475-476.

26. Silverstein, R. M.; Webster, F. X. Spectrometric Identification of Organic Compounds; sixth ed.; John Wiley \& Sons: New York, 1998, p 212.

27. Mukai, C.; Sugimoto, Y.; Ikeda, Y.; Hanaoka, M. Chem. Commun. 1994, 1161-1162.

28. Uenishi, J.; Sohma, A.; Yonemitsu, O. Chem. Lett. 1996, 595-596.

29. Bernstein, M. A.; Morton, H. E.; Guindon, Y. J. Chem. Soc., Perkin Trans. 2 1986, 1155-1163. 


\section{Analytical Data}

A. GC traces and ${ }^{1} \mathrm{H}$ NMR spectra of unpurified isomer ratios

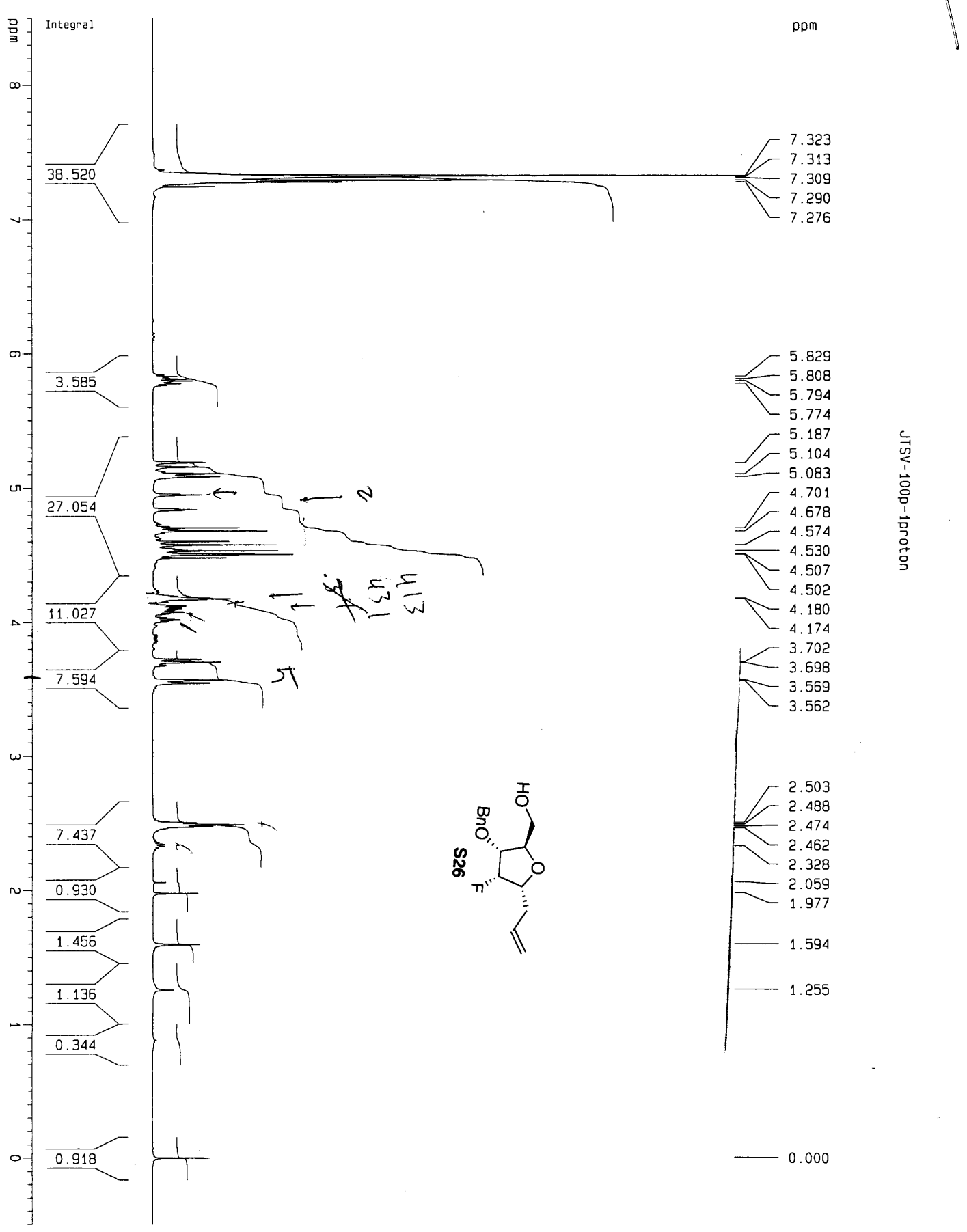




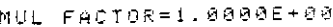

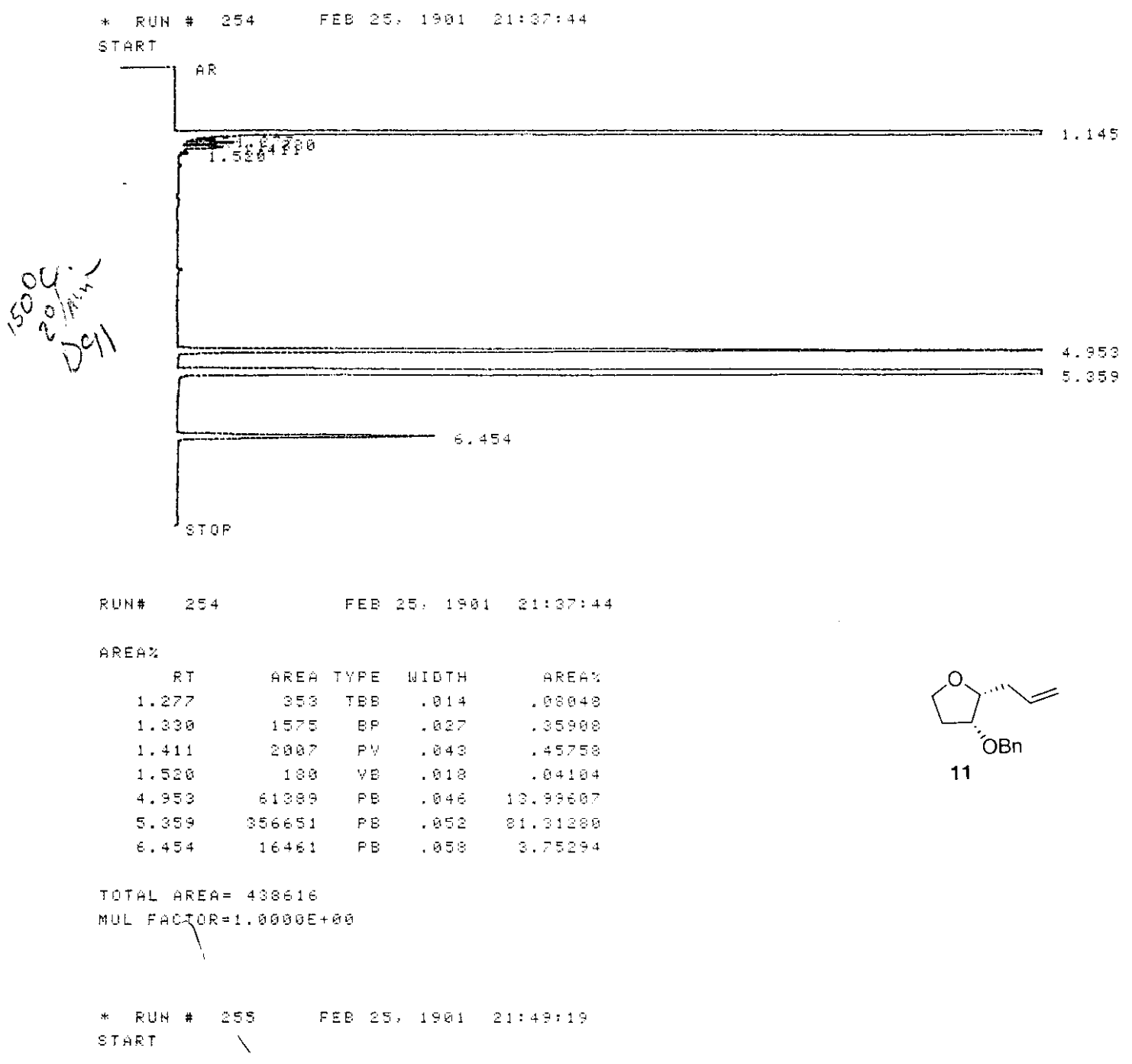




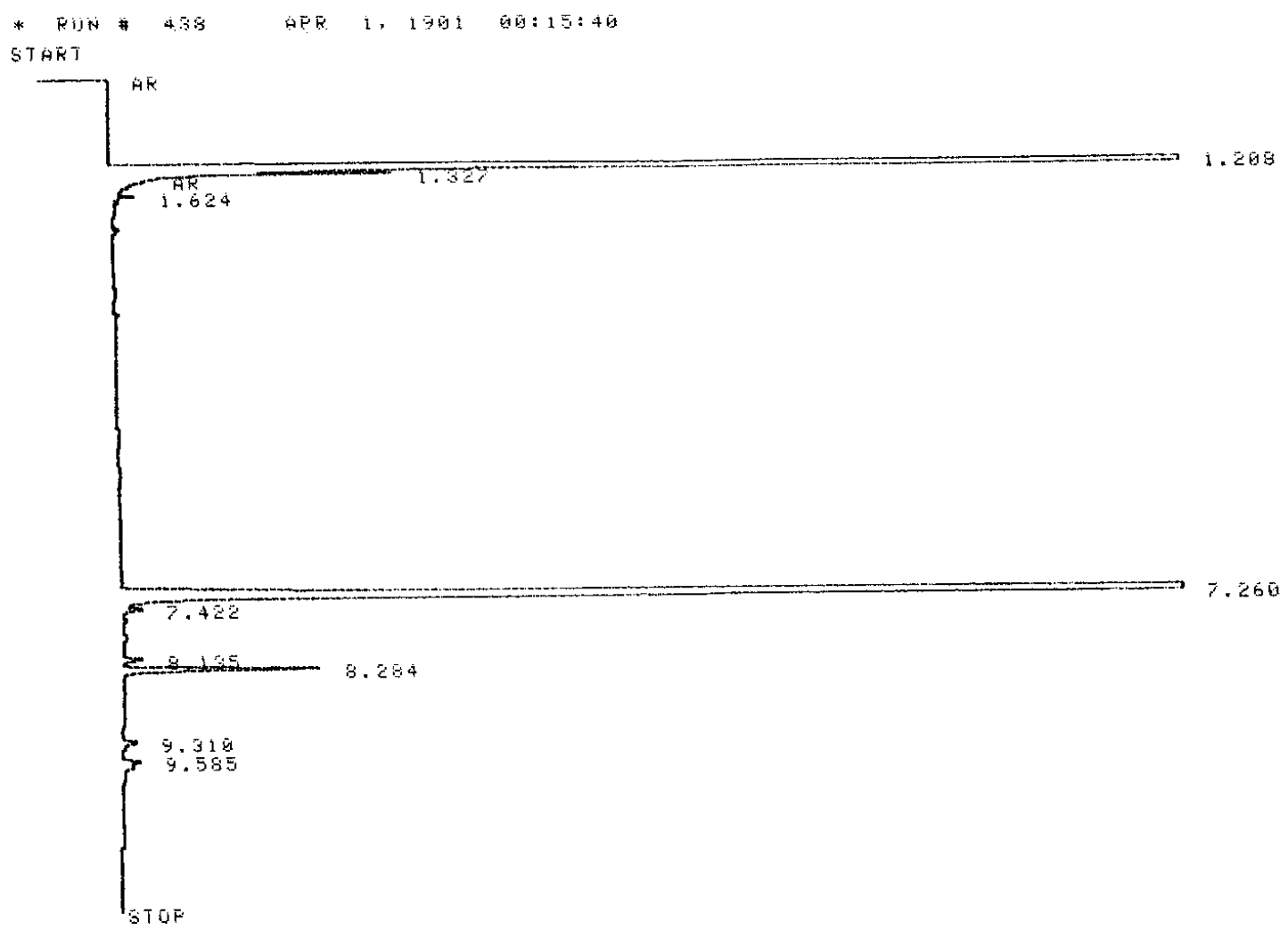

\begin{tabular}{|c|c|c|c|c|}
\hline $\mathrm{RUN} \#$ & & $A F F$ & $1, \quad 15 \overline{1} 1$ & 6日: $15: 46$ \\
\hline \multicolumn{5}{|l|}{ AEEA\% } \\
\hline RT & $A F E A$ & TYFE & WIOTH & AREM\% \\
\hline 1.624 & 137 & $B F$ & .017 & .95437 \\
\hline 7.256 & 245554 & $F E$ & .044 & 47.44557 \\
\hline 7.422 & 244 & $\overline{B F}$ & .035 & .59683 \\
\hline 8.135 & 513 & Wu & .054 & .29350 \\
\hline$\therefore .284$ & 4532 & Qu & .651 & 1.75848 \\
\hline 9.310 & 465 & $B F$ & .066 & .18493 \\
\hline 9.535 & 545 & $F$ & .061 & .21628 \\
\hline
\end{tabular}

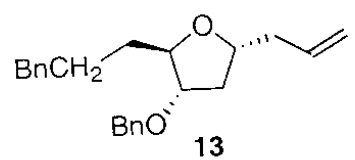

THTAL AKEM $=251991$

MUL FACTOR=1. 

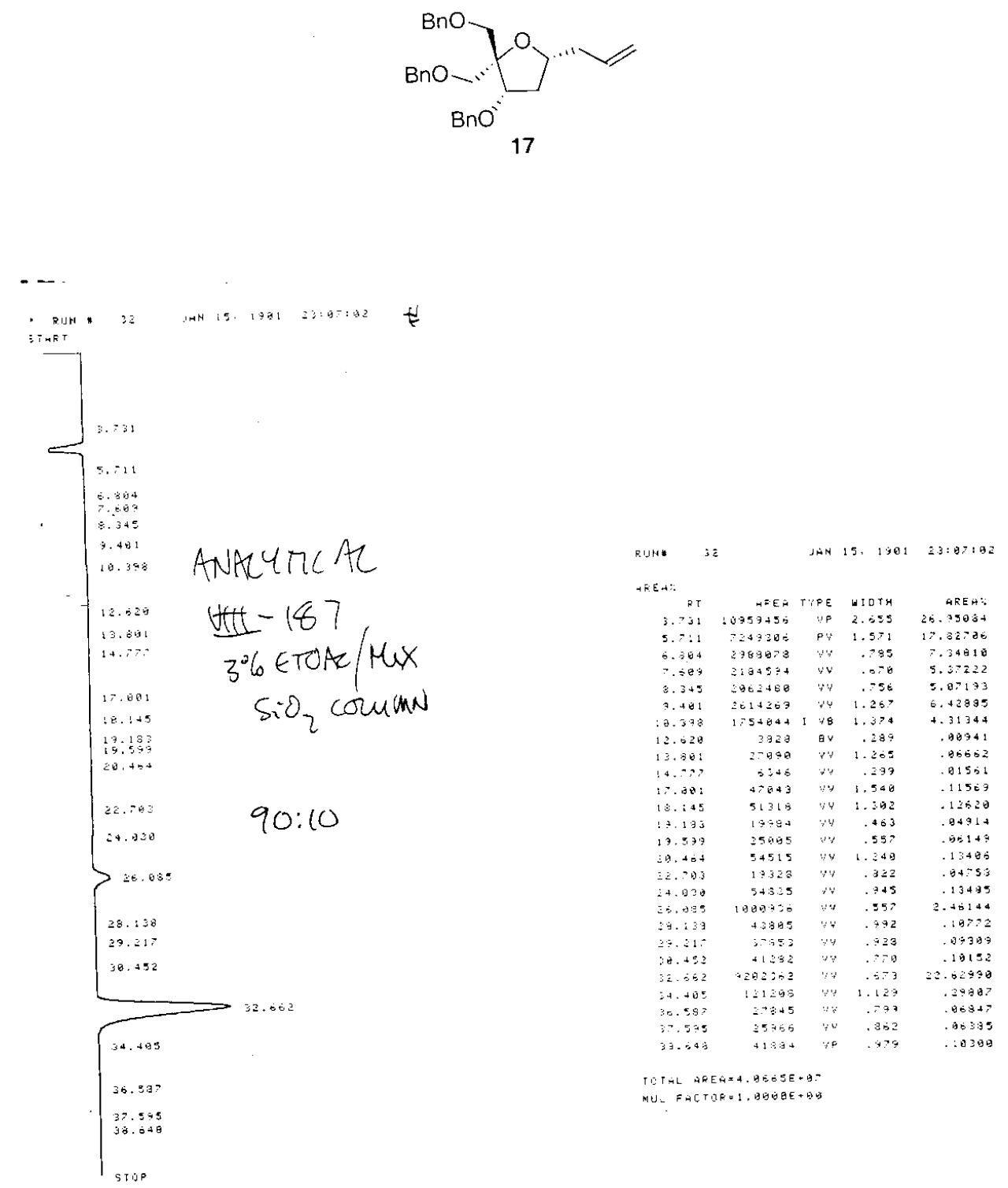


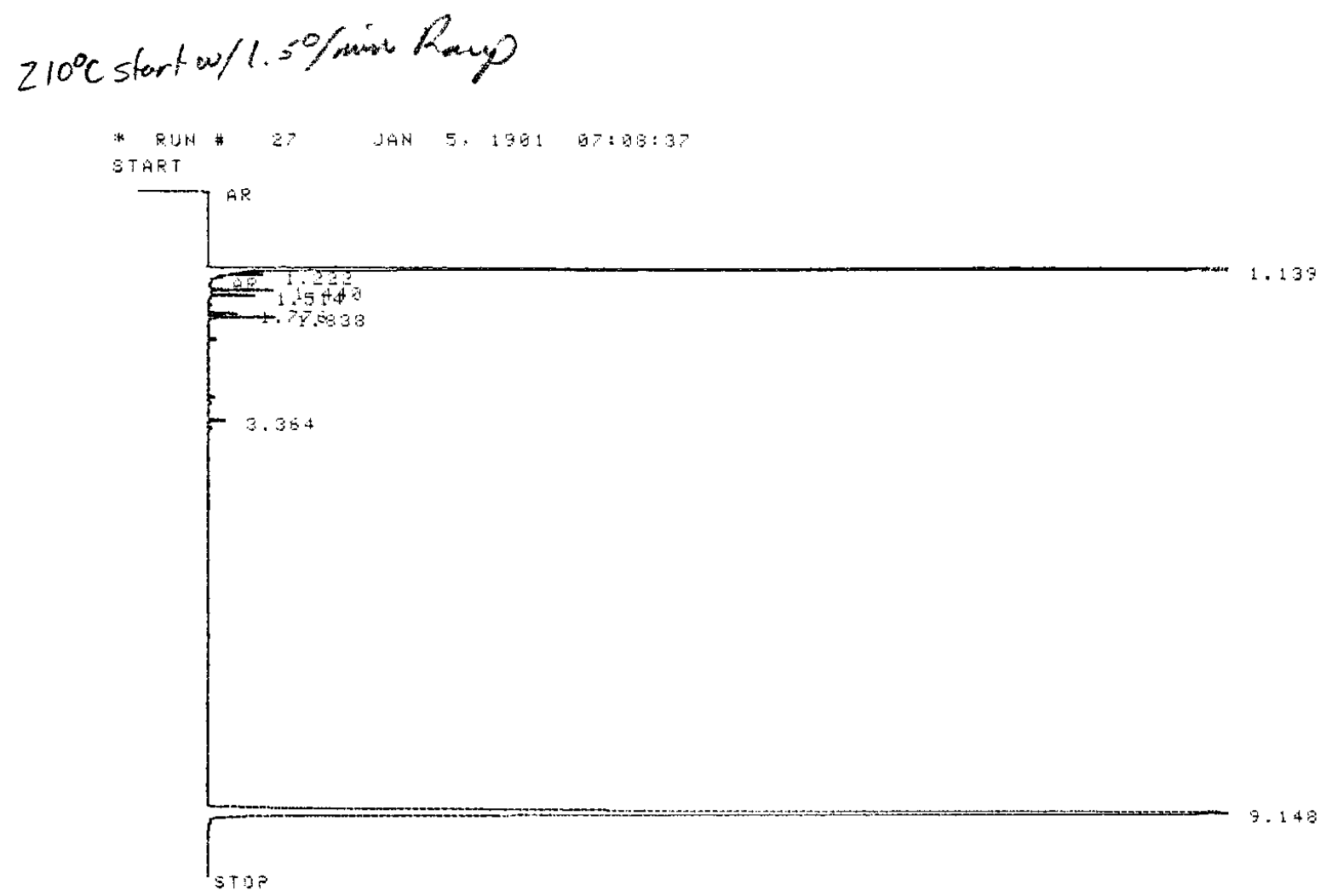

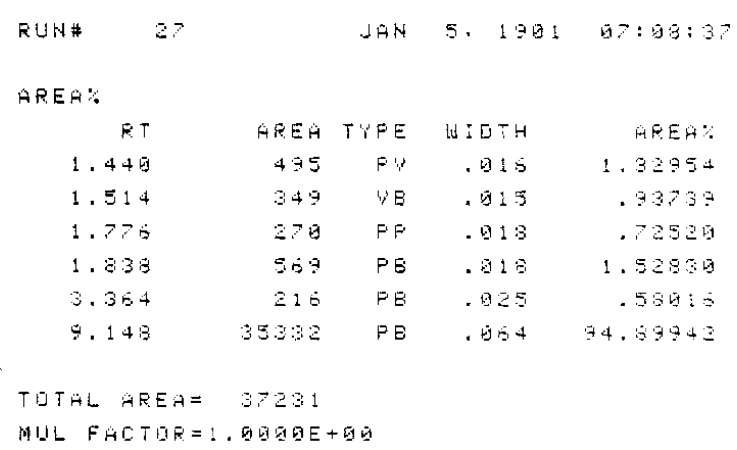

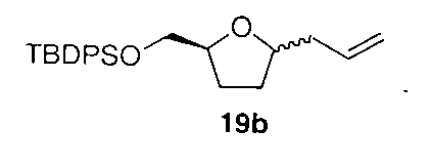




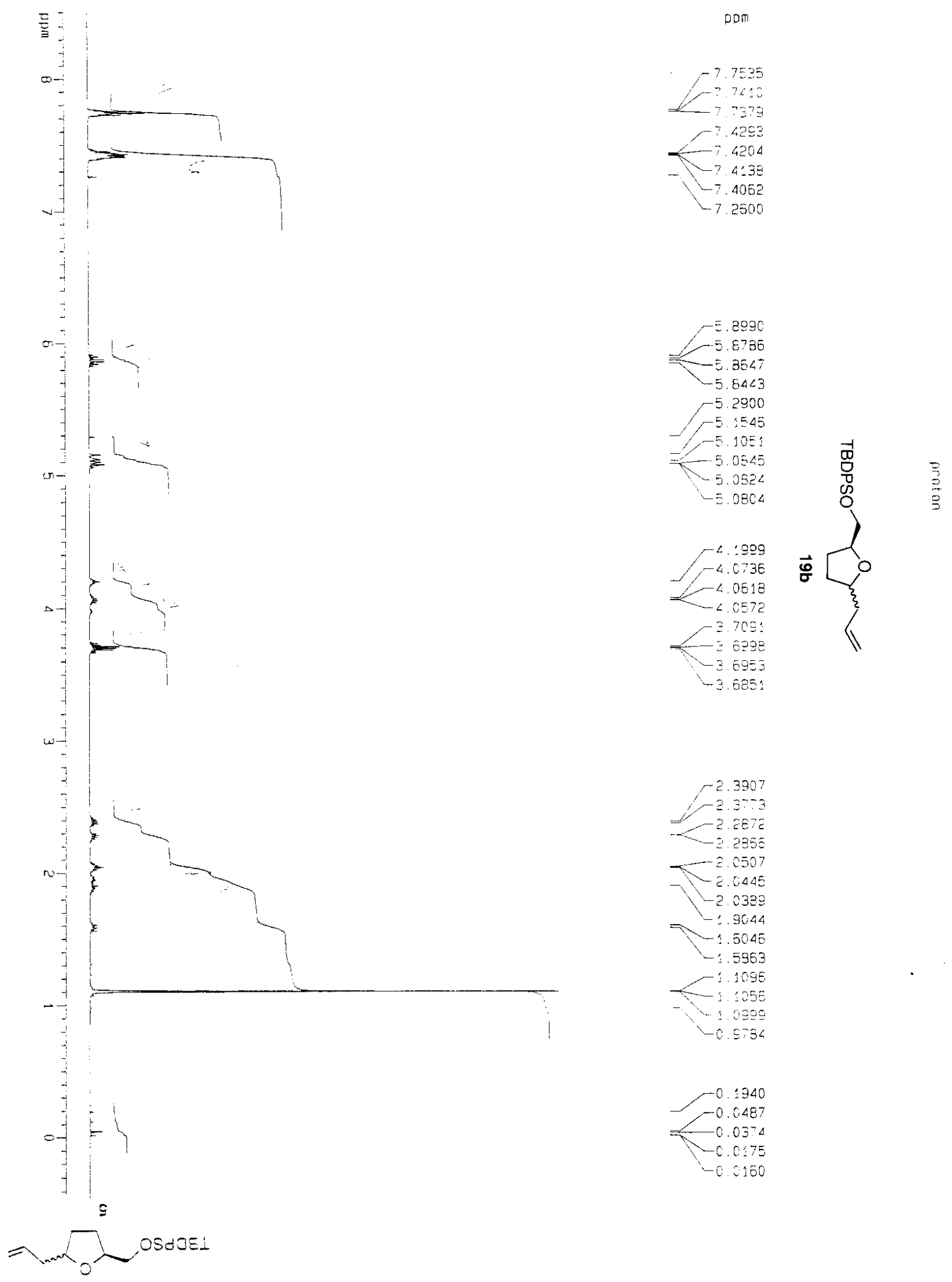




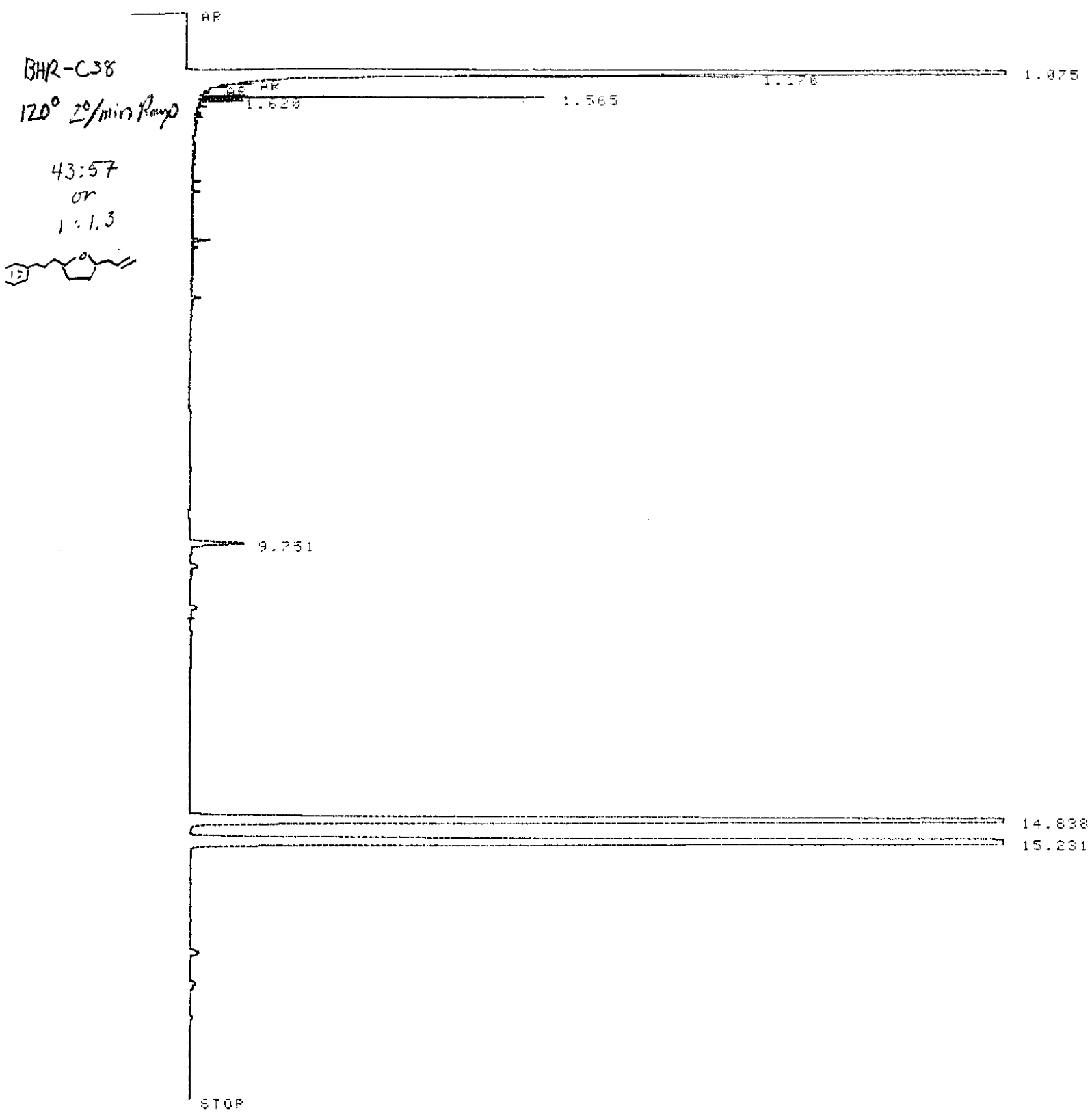

\begin{tabular}{|c|c|c|c|c|}
\hline RULH & & FEE: & $15 . \quad 1905$ & $92: 94: 28$ \\
\hline \multicolumn{5}{|l|}{ AFEA } \\
\hline $\mathrm{RT}$ & $A P E A$ & TYPE & WI D TH & AFEA: \\
\hline 1.565 & 1349 & $F F$ & .813 & 1. 2712 \\
\hline 1.68 & $2: 9$ & $F$ & .517 & .20530 \\
\hline 9.751 & 1058 & E.E & . 105 & $.99-15$ \\
\hline 14.830 & 44588 & FE & .015 & 42.62455 \\
\hline 15.231 & 58896 & E: & .81 & 55.50487 \\
\hline
\end{tabular}

TOTAL APEA = 100119

MLIL FACTQR=1. GEEEE+GG

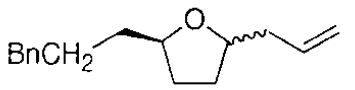

$19 c$ 


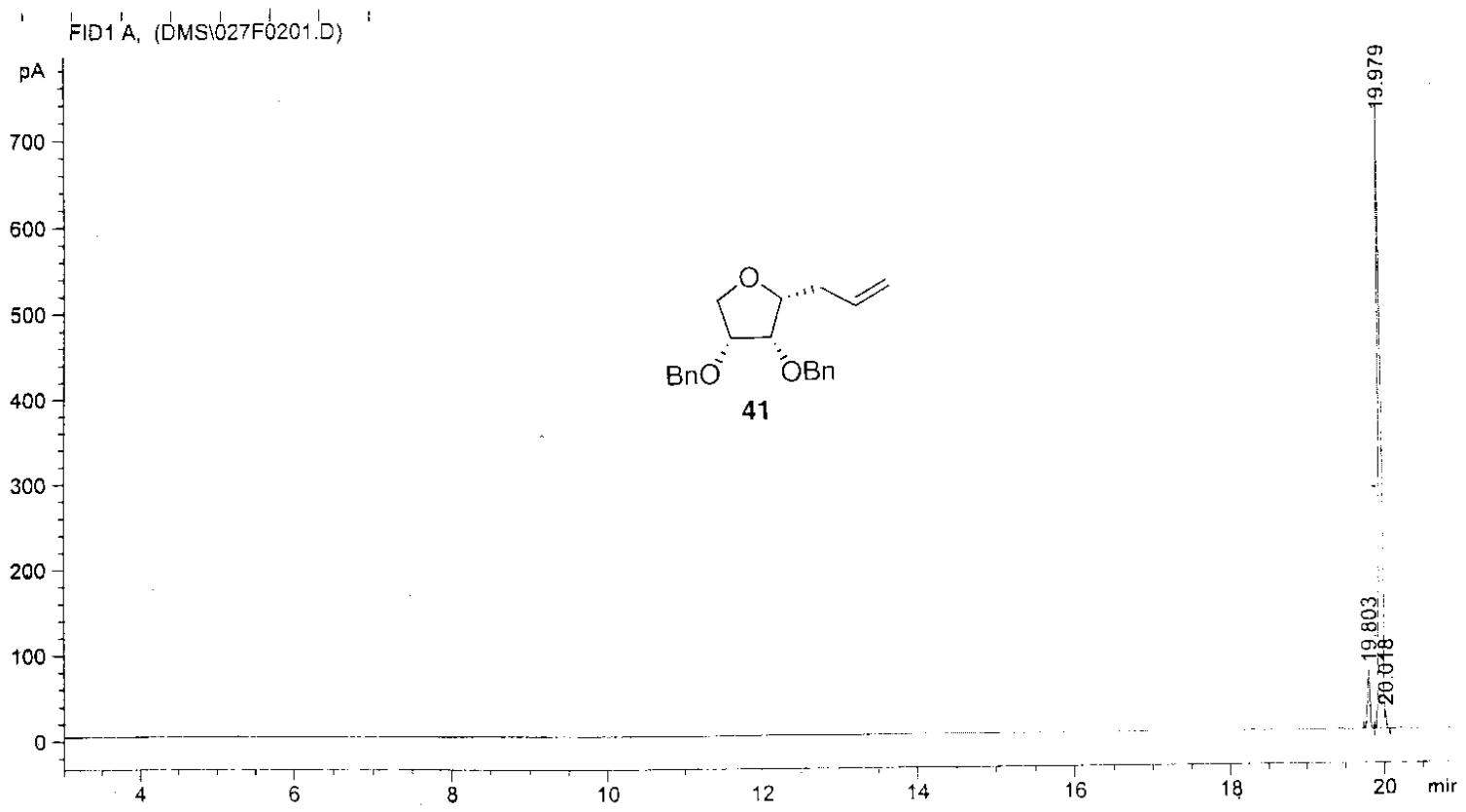

\begin{tabular}{|c|c|c|c|c|c|}
\hline $\begin{array}{c}\text { Peak } \\
\#\end{array}$ & $\begin{array}{c}\text { Retiime } \\
\text { [min] }\end{array}$ & Type & $\begin{array}{l}\text { Width } \\
\text { [min] }\end{array}$ & $\begin{array}{r}\text { Area } \\
{\left[p A^{*} \mathrm{~s}\right]}\end{array}$ & $\begin{array}{c}\text { Area } \\
\frac{a}{6}\end{array}$ \\
\hline & & & & $\ldots \ldots$ & $\ldots \ldots$ \\
\hline 1 & 0.685 & $B P$ & $4.91 e-3$ & 1.35592 & 0.00117 \\
\hline 2 & 0.707 & VV & $6.34 e-3$ & 13.85377 & 0.01195 \\
\hline 3 & 0.756 & VV & $7.38 e-3$ & 141.97148 & 0.12243 \\
\hline 4 & 0.790 & & 0.0000 & 0.00000 & 0.00000 \\
\hline 5 & 0.830 & VV & $6.88 e-3$ & 2.92838 & 0.00253 \\
\hline 6 & 0.855 & & 0.0000 & 0.00000 & 0.00000 \\
\hline 7 & 0.879 & VB S & 0.0227 & $1.13639 e 5$ & 97.99864 \\
\hline 8 & 0.914 & BB $x$ & $7.12 e-3$ & 16.42007 & 0.01416 \\
\hline 9 & 1.119 & VB $\mathrm{X}$ & $8.54 e-3$ & 4.40571 & 0.00380 \\
\hline 10 & 1.217 & $B P$ & $9.80 e-3$ & 8.82983 & 0.00761 \\
\hline 11 & 1.679 & $\mathrm{~PB}$ & 0.0142 & 21.35434 & 0.01842 \\
\hline 12 & 19.803 & $\mathrm{BV}$ & 0.0283 & 150.58685 & 0.12986 \\
\hline 13 & 19.979 & VV & 0.0310 & 1920.46521 & 1.65615 \\
\hline 14 & 20.018 & VB & 0.0276 & 27.39649 & 0.02363 \\
\hline 15 & 21.133 & $\mathrm{VB}$ & 0.0299 & 11.20213 & 0.00966 \\
\hline
\end{tabular}

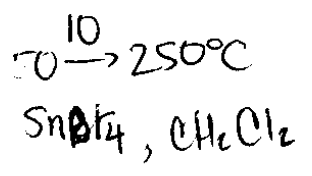




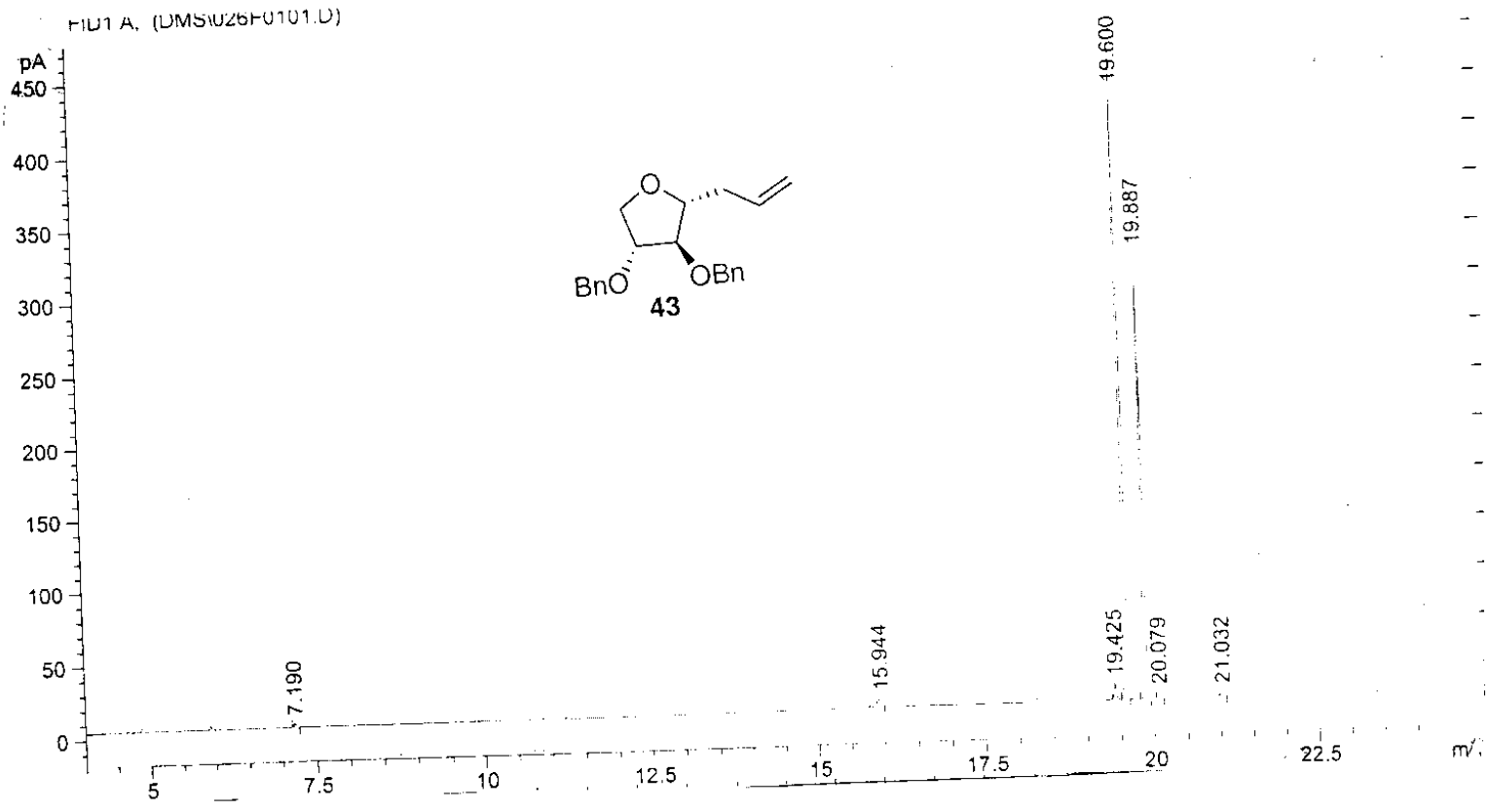

\begin{tabular}{|c|c|c|c|c|c|c|}
\hline eak & RetTime & Type & $\begin{array}{c}\text { width } \\
{[\mathrm{min}]}\end{array}$ & $\begin{array}{r}\text { Area } \\
{[p A * S]}\end{array}$ & $\begin{array}{c}\text { He } \text { y:2t } \\
\text { ph: }\end{array}$ & $\begin{array}{c}\text { Area } \\
\frac{\circ}{6}\end{array}$ \\
\hline & $-\cdots$ & & - . - & & & \\
\hline 11 & 19.425 & BV & 0.0275 & 25.40044 & 21.86240 & 0.01959 \\
\hline 12 & 19.600 & VV & 0.0294 & 984.28204 & 448.51312 & 0.75916 \\
\hline 13 & 19.887 & $\mathrm{VB}$ & 0.0257 & 640.65869 & 309.09216 & 0.49413 \\
\hline 14 & 20.079 & VB & 0.0267 & 11.94623 & 5.57966 & 0.00921 \\
\hline 15 & 21.032 & $\mathrm{BB}$ & 0.0265 & 9.76351 & 4.50729 & 0.00753 \\
\hline
\end{tabular}




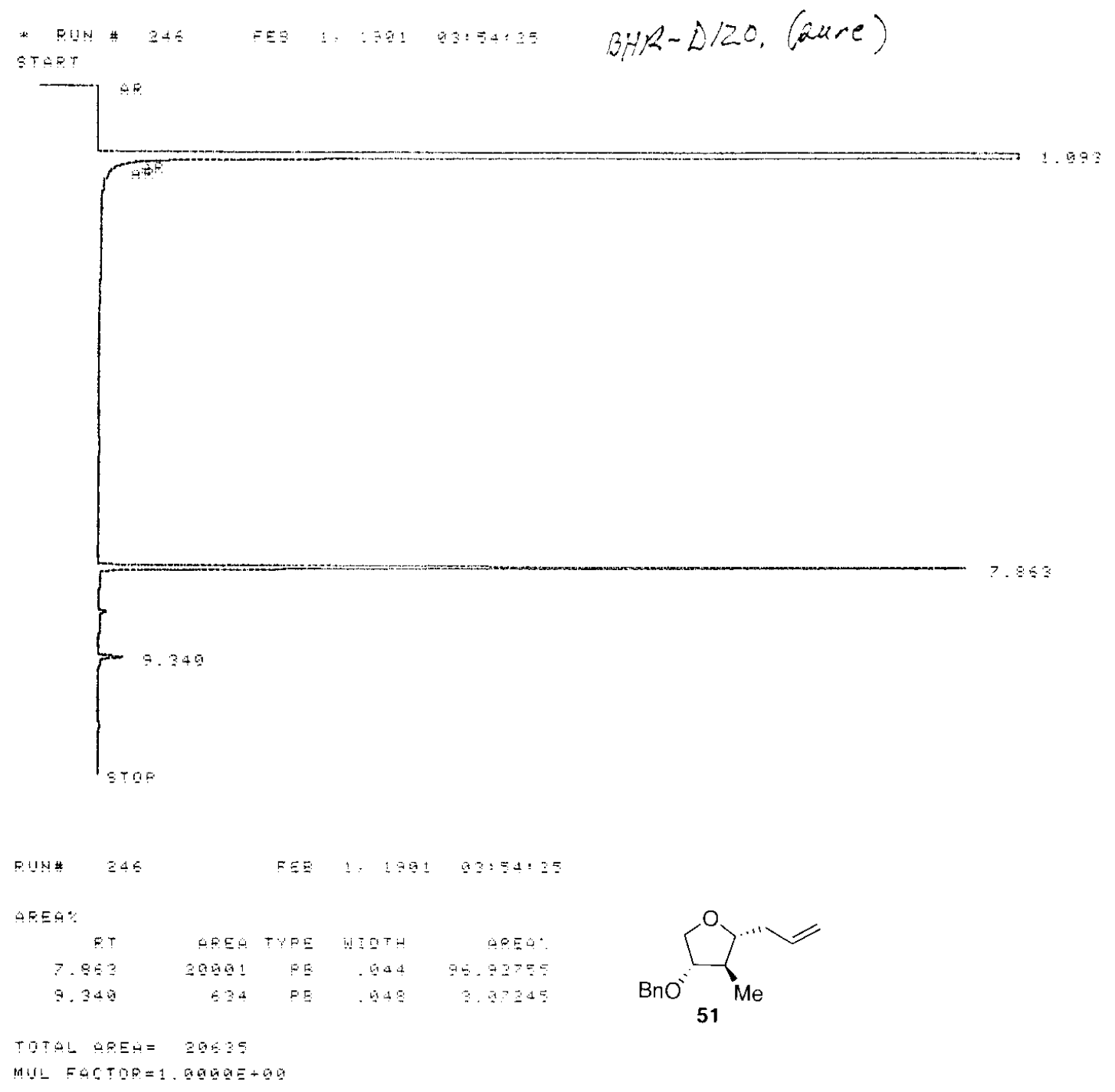




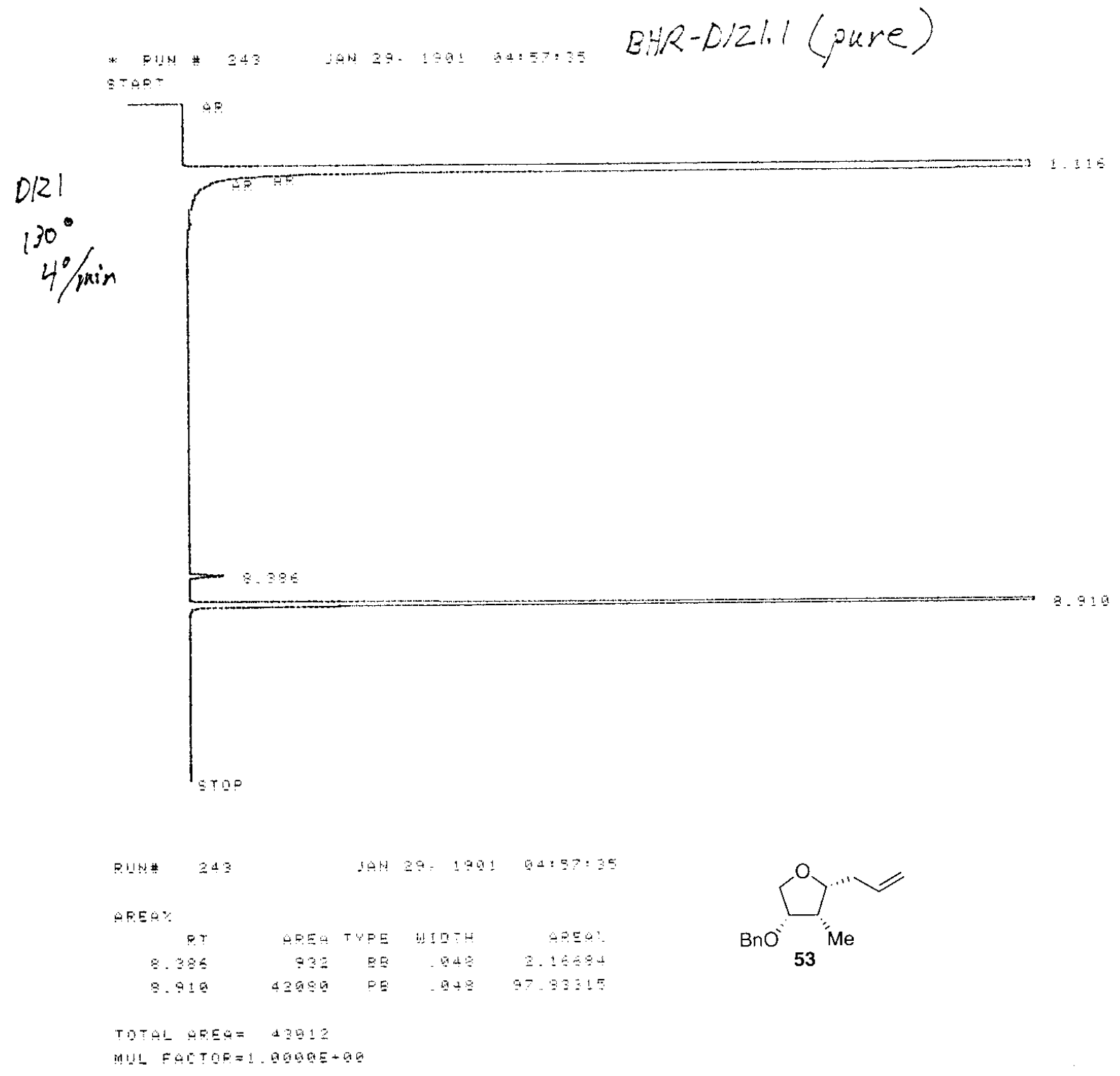




\section{B. Selected ${ }^{1}$ H NMR spectra}
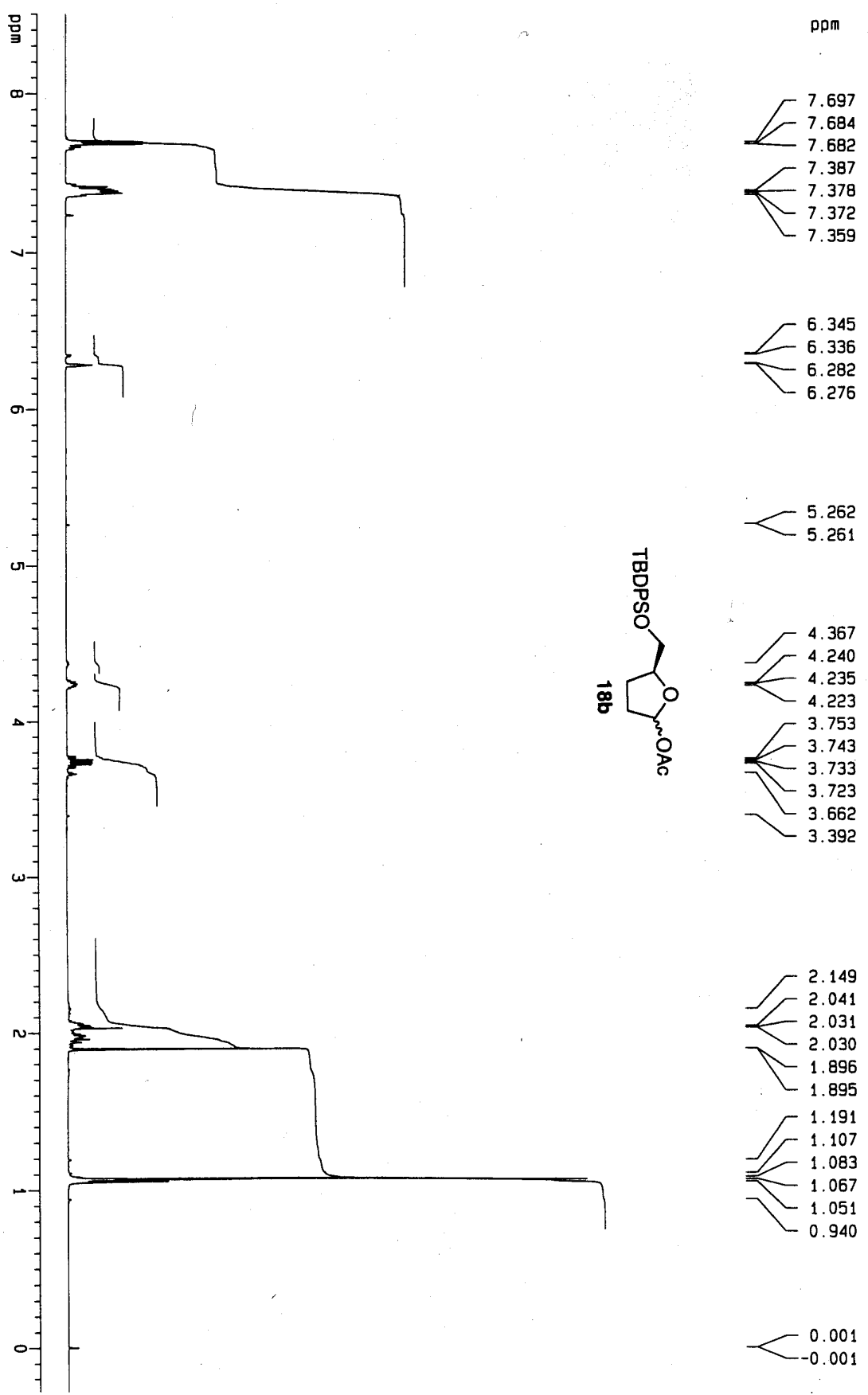


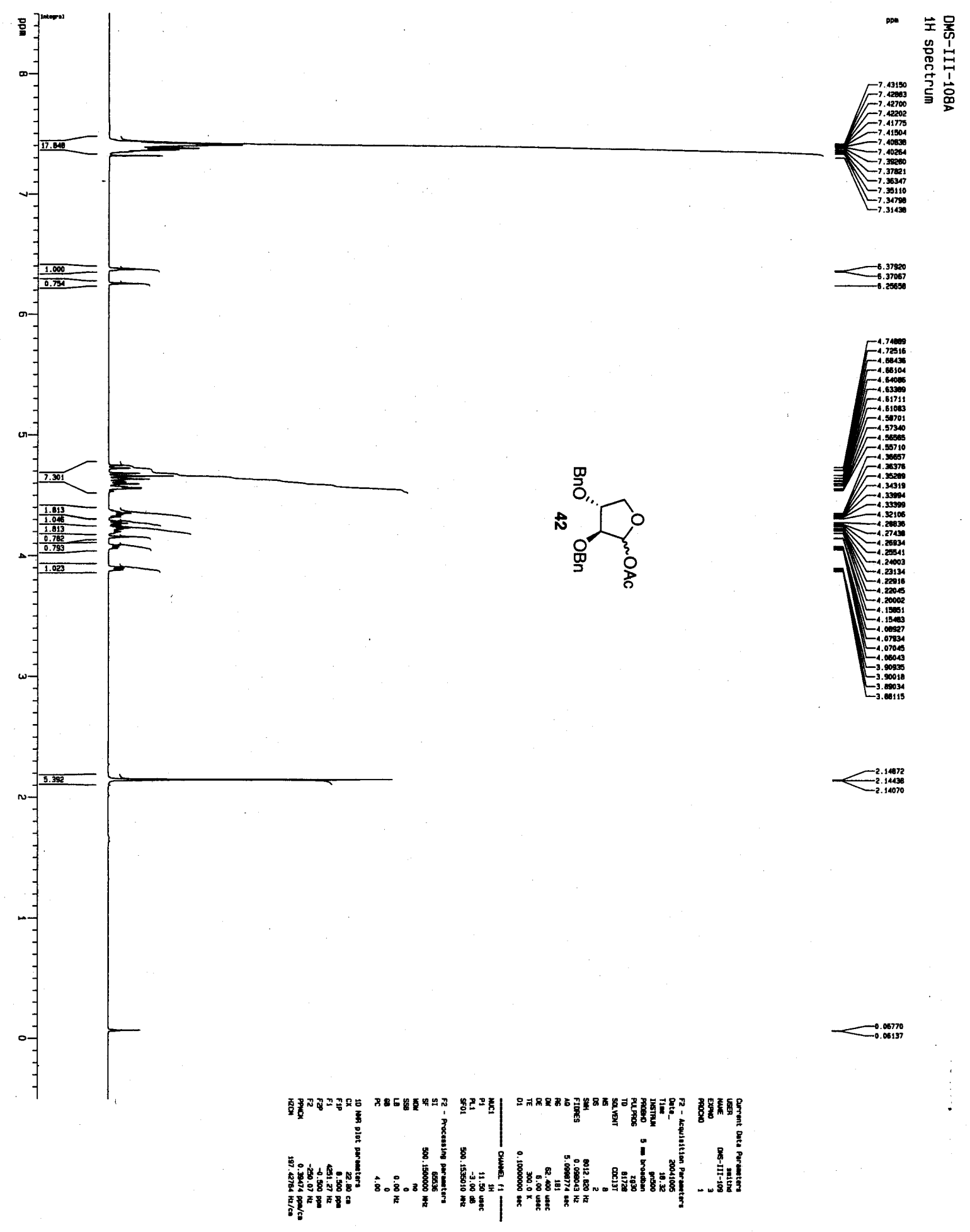




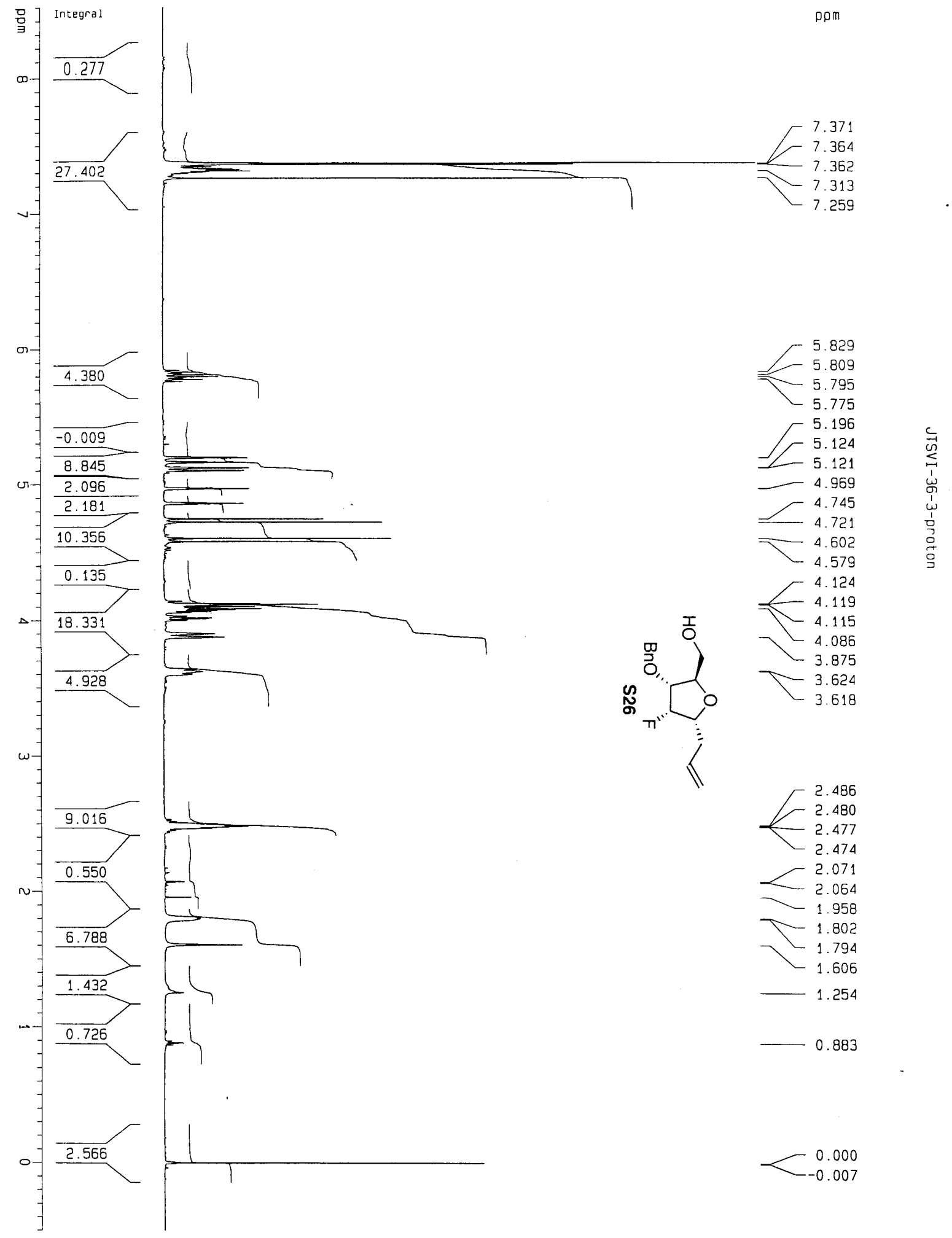




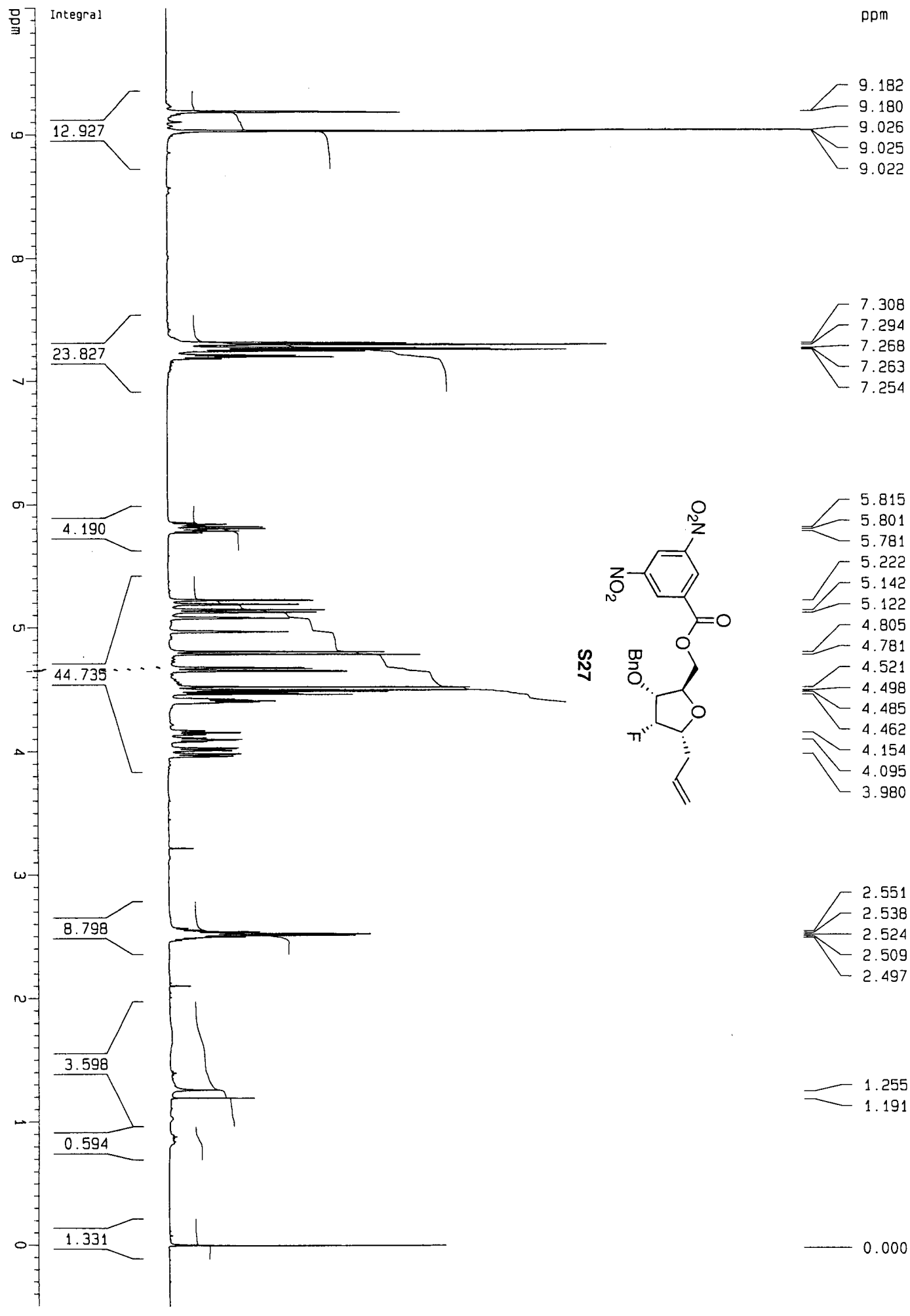




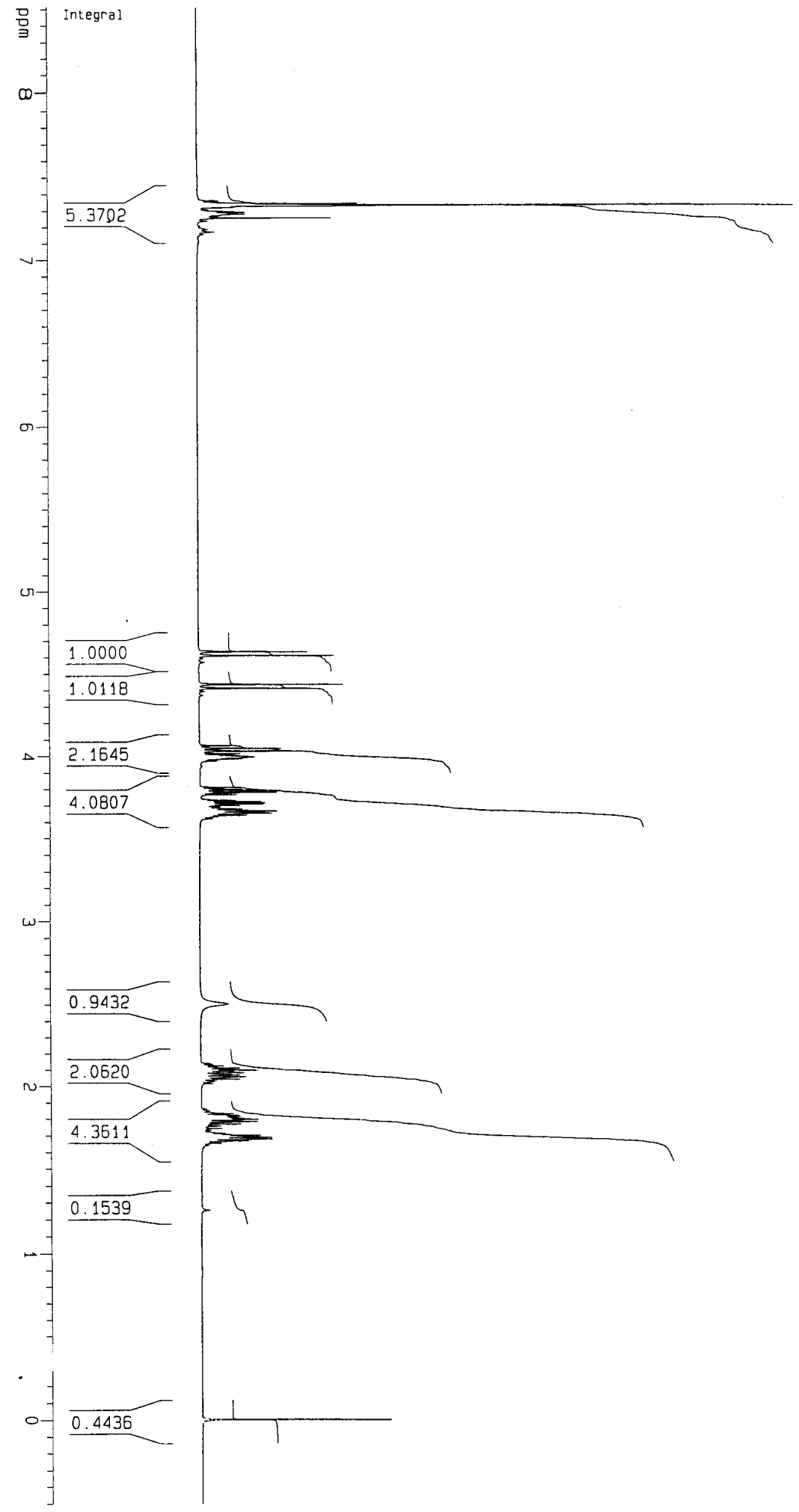

ppm
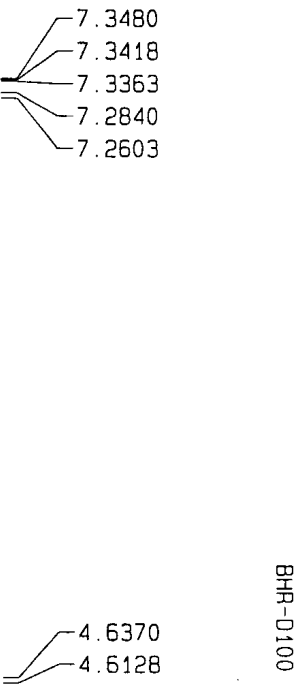

$-4.6128$

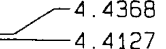

4.0488
-4.0327

$\begin{array}{r}3.7954 \\ -3.7850 \\ \hline\end{array}$

$=3.7190$

$-3.6704$

$-3.6568$

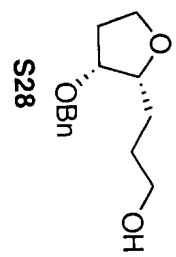

$-2.5061$

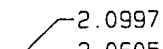

$-2.0605$

$-1.8010$

$-1.6942$

1.6909
1.6831

$-1.6796$

$-1.2542$

$-0.0000$ 


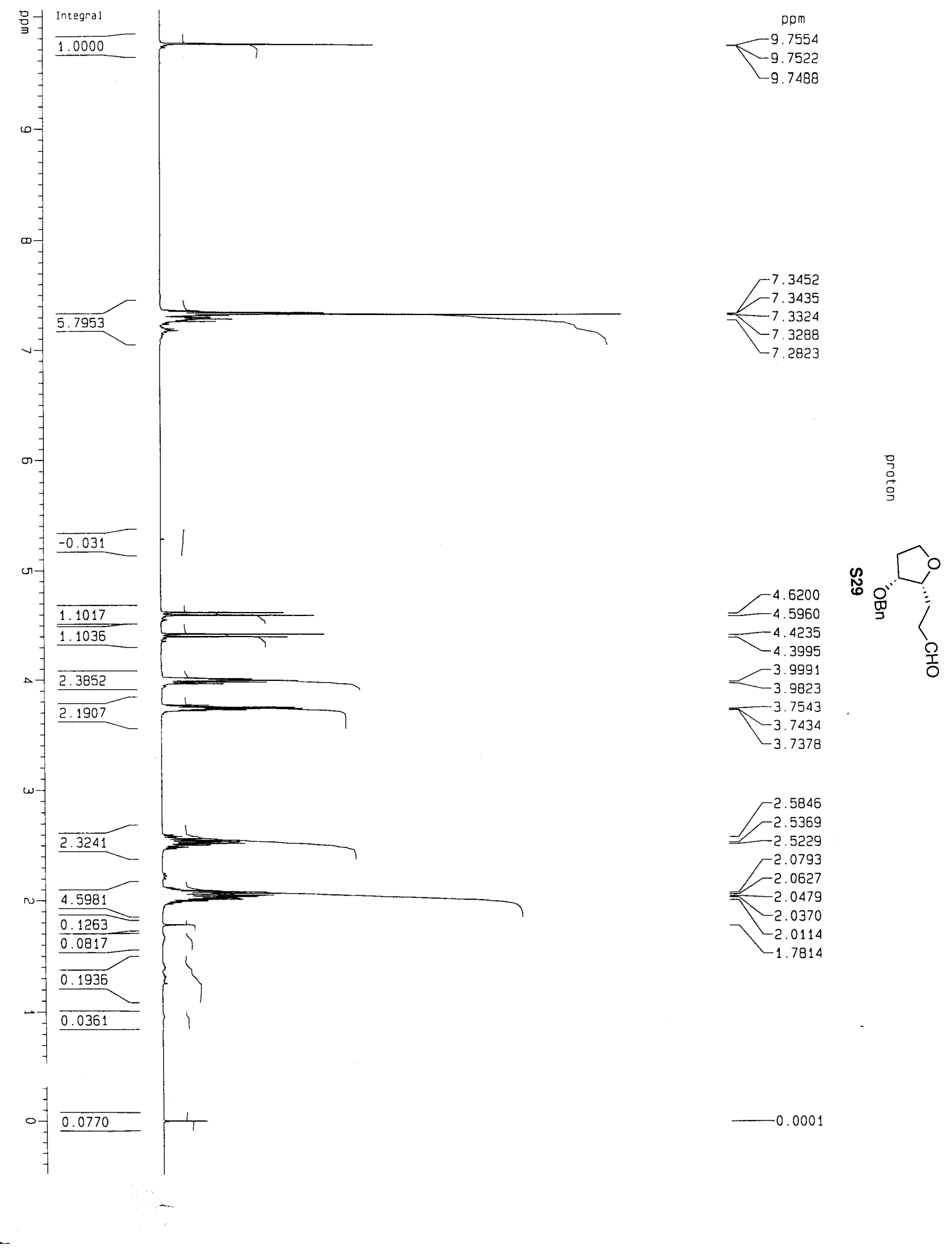




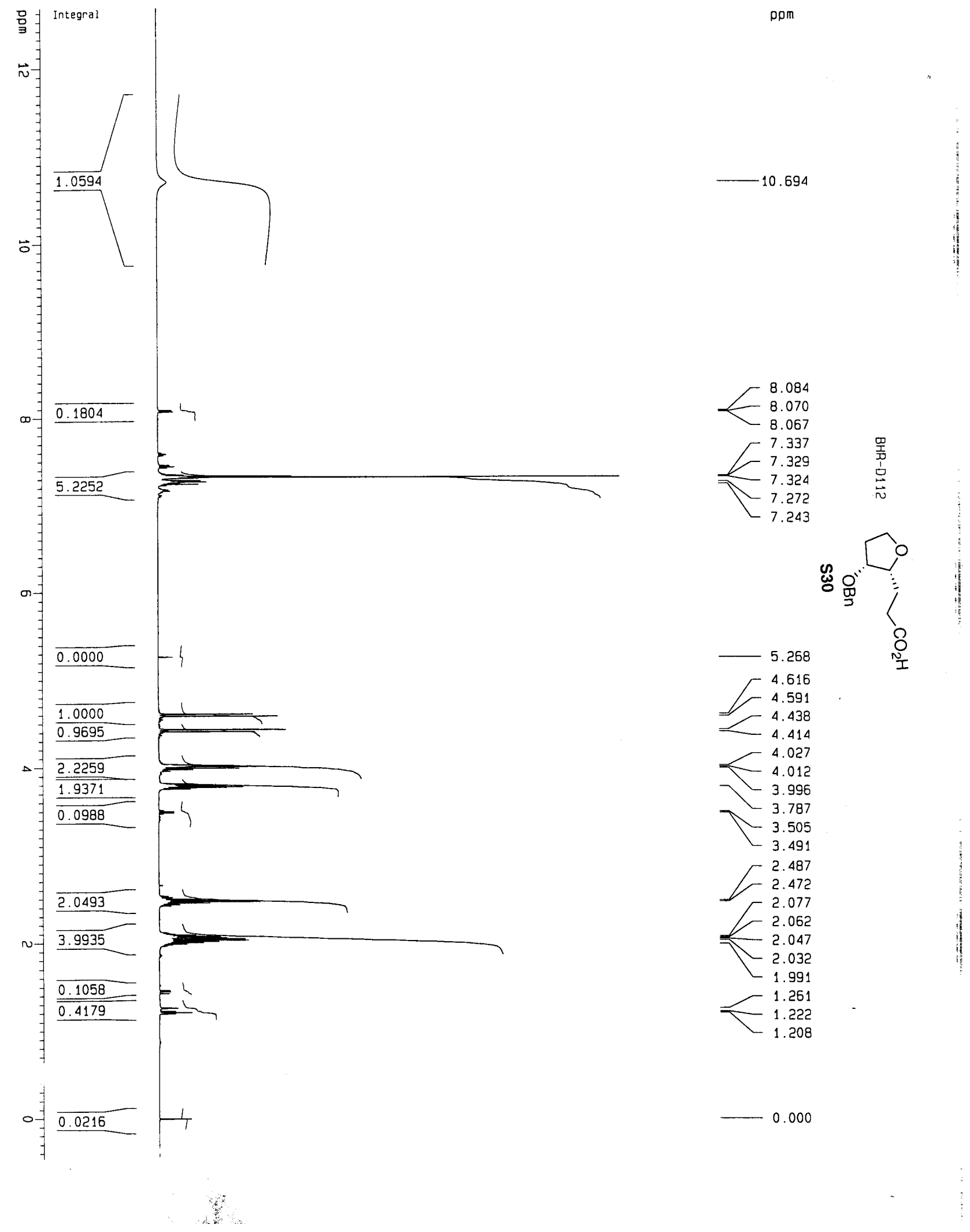




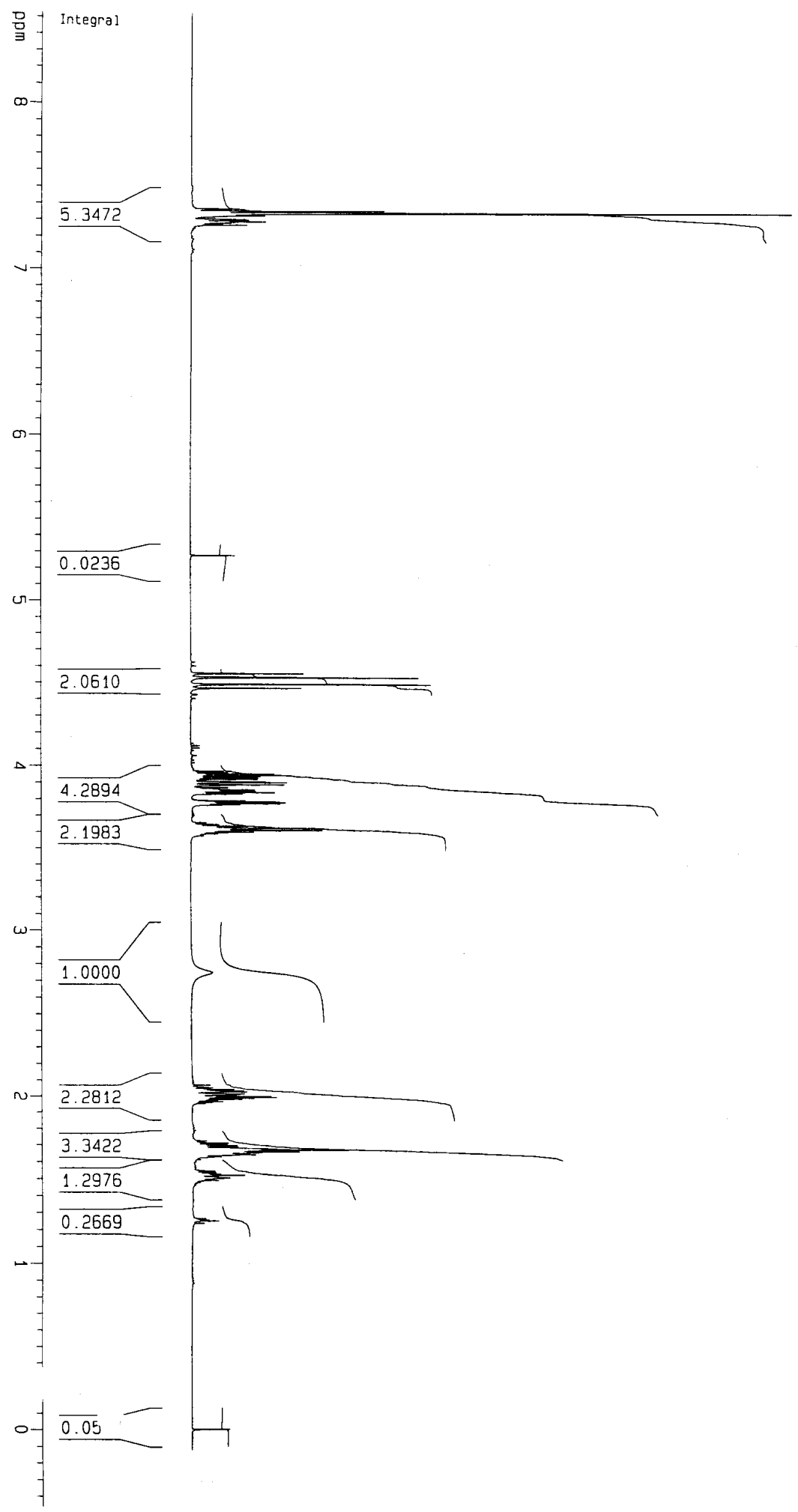

ppm
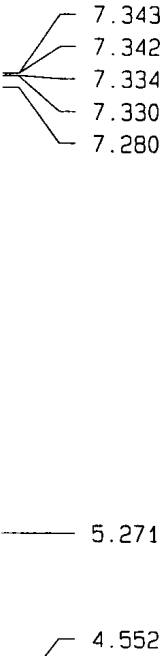

.552

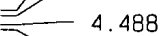

3.948

$-3.941$

$=3.898$

$-3.885$

$-3.775$

3.619

3.607

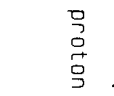

赵

2.746

Г 1.997

$-1.991$

1.985

1.676

$\sim 1.672$

$\checkmark \begin{array}{r}1.667 \\ 1.657\end{array}$

1.503

1.249

0.000 


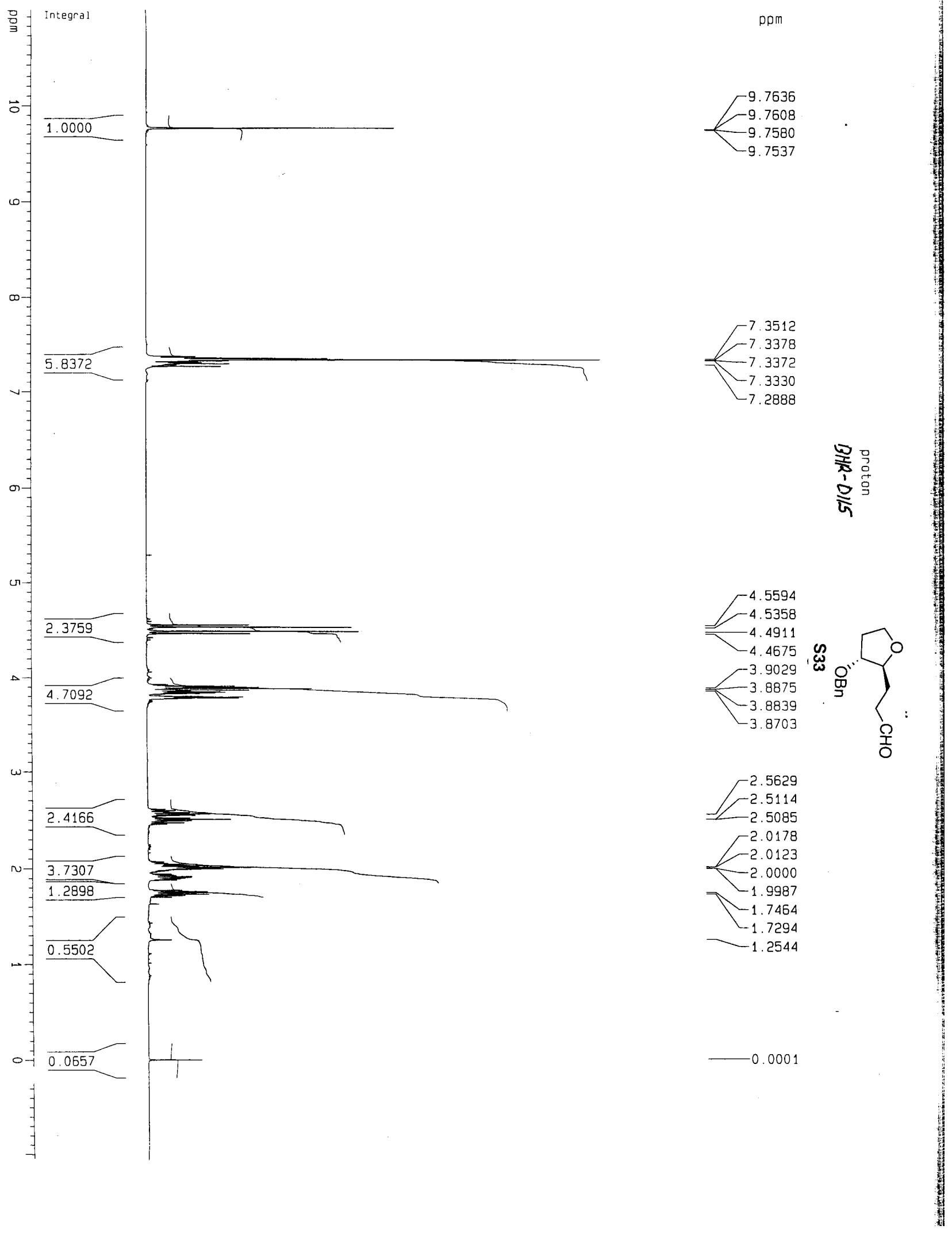




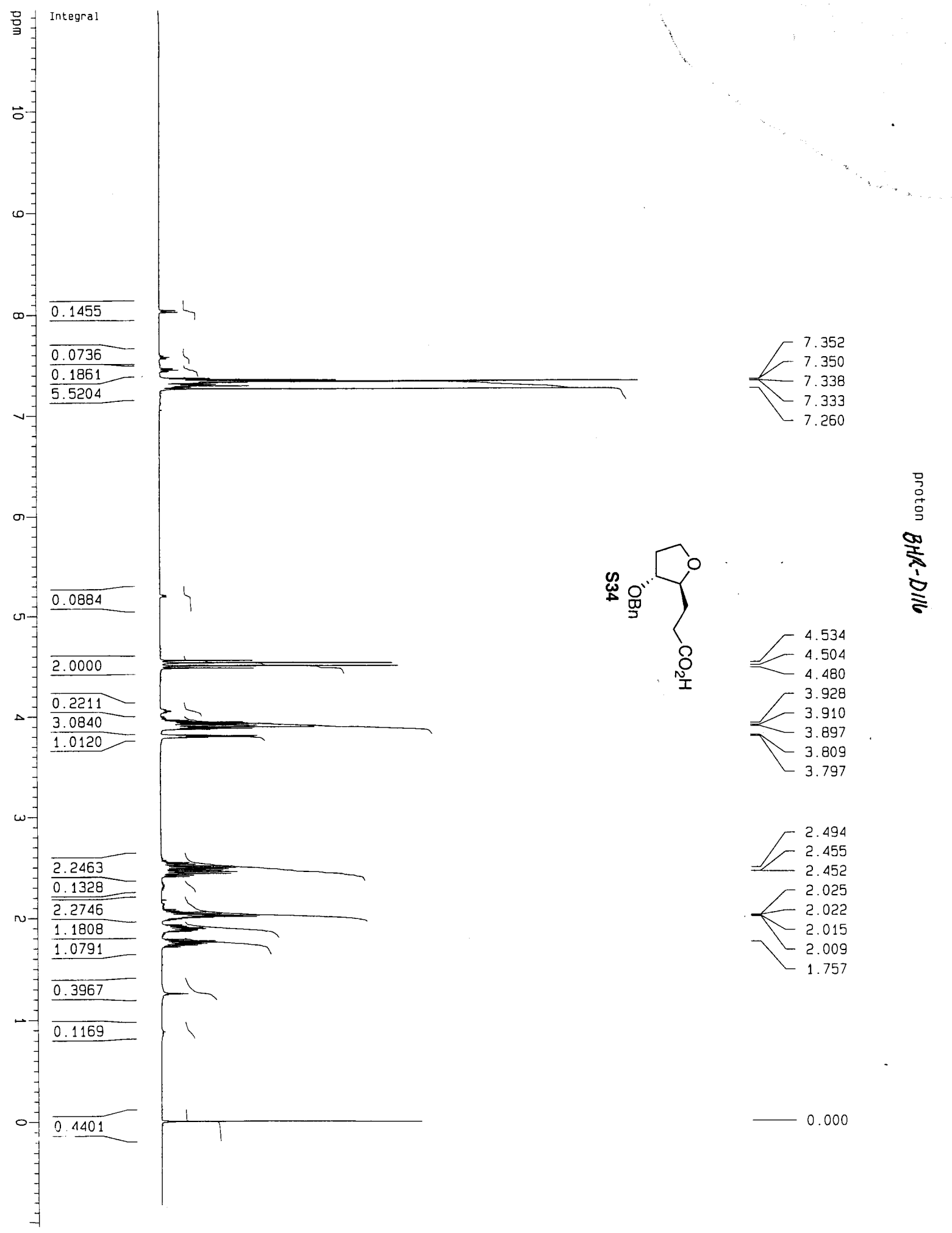




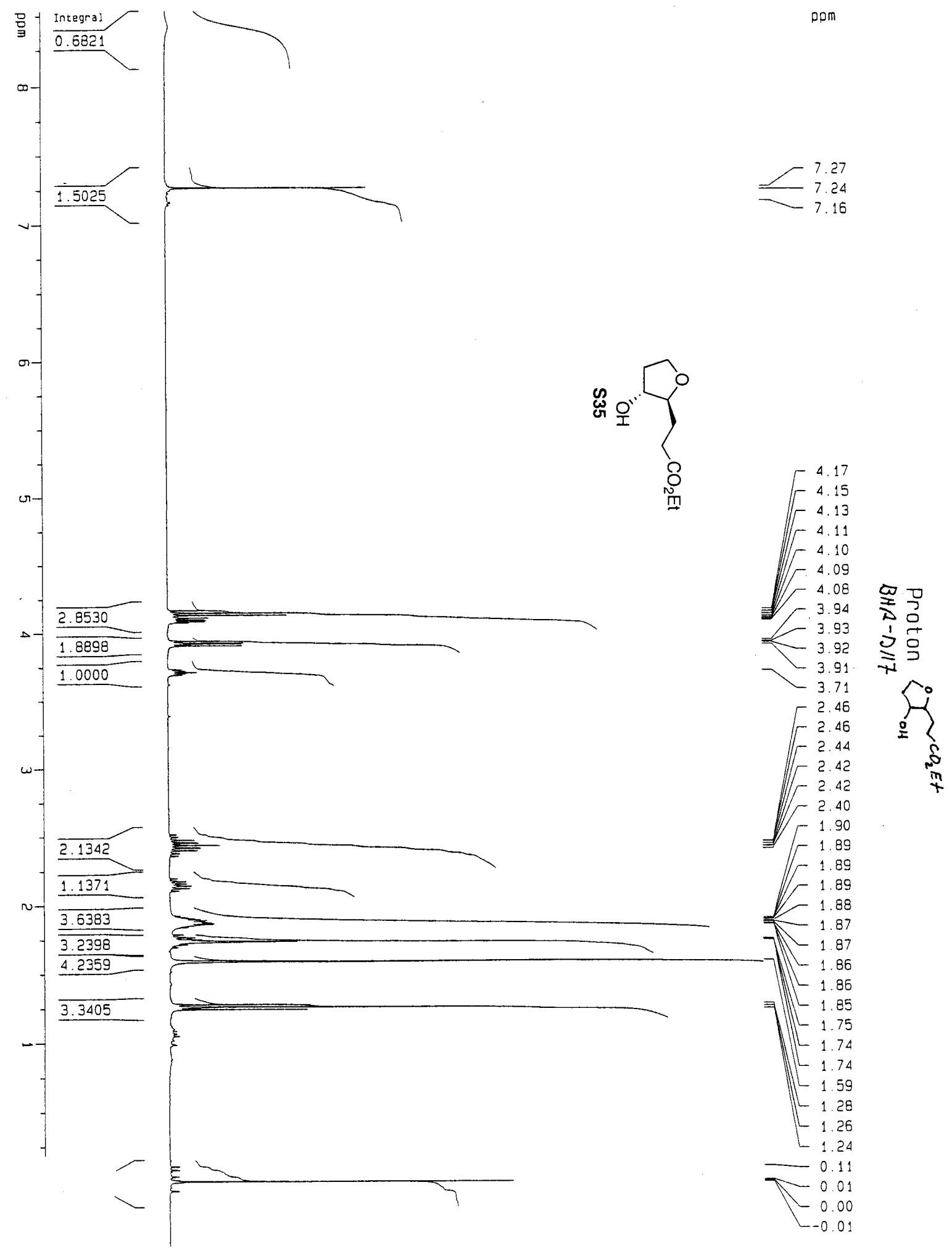




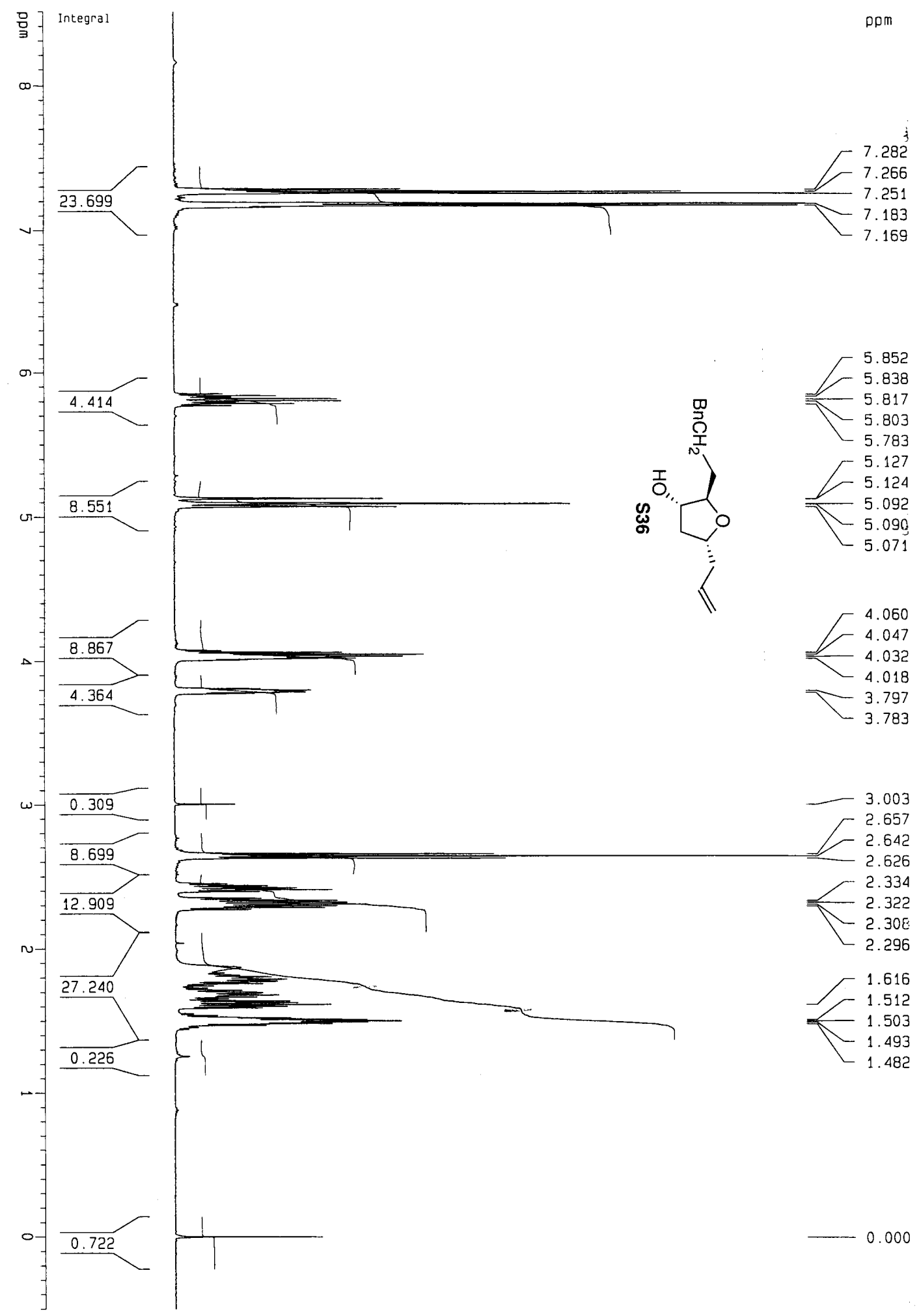




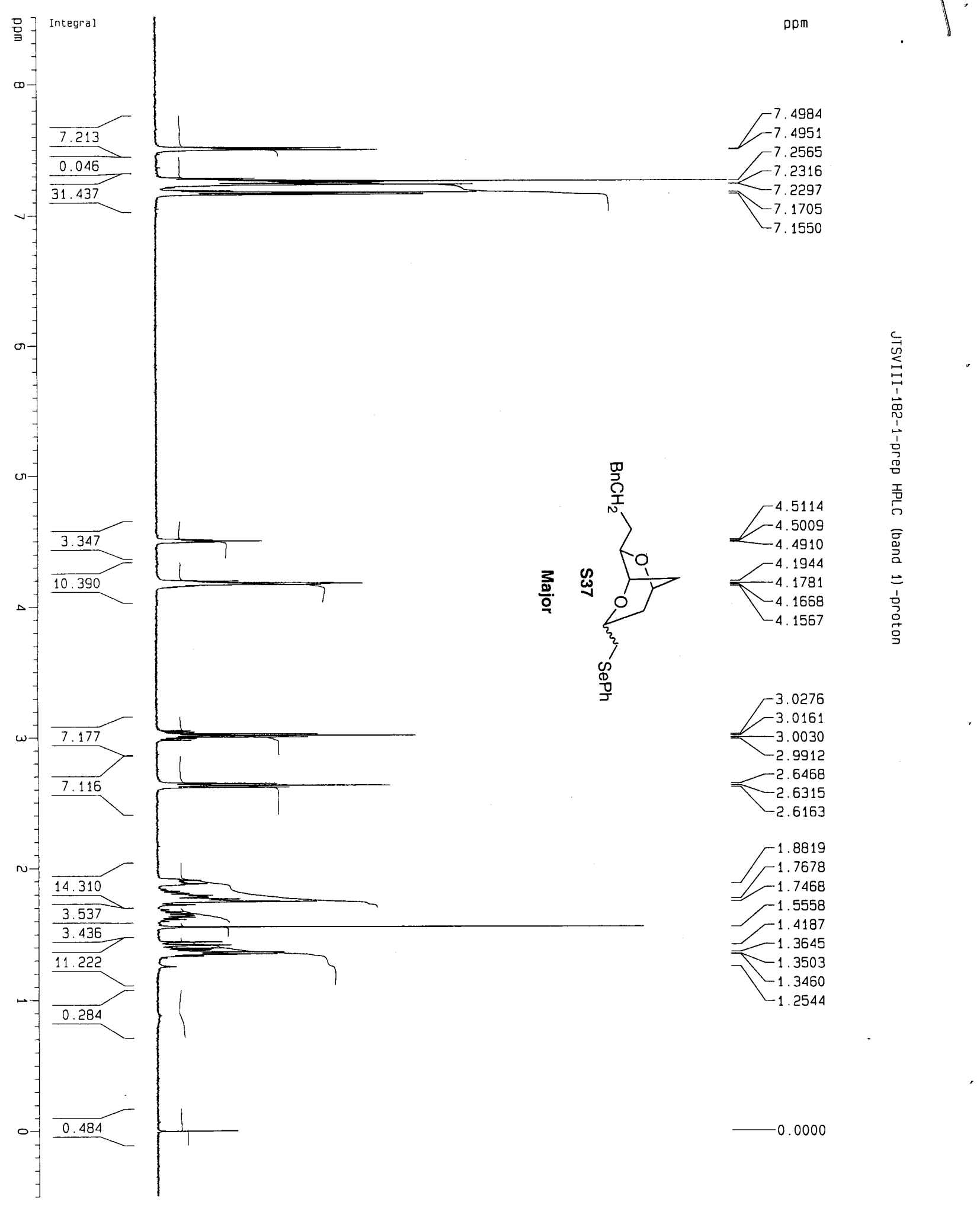




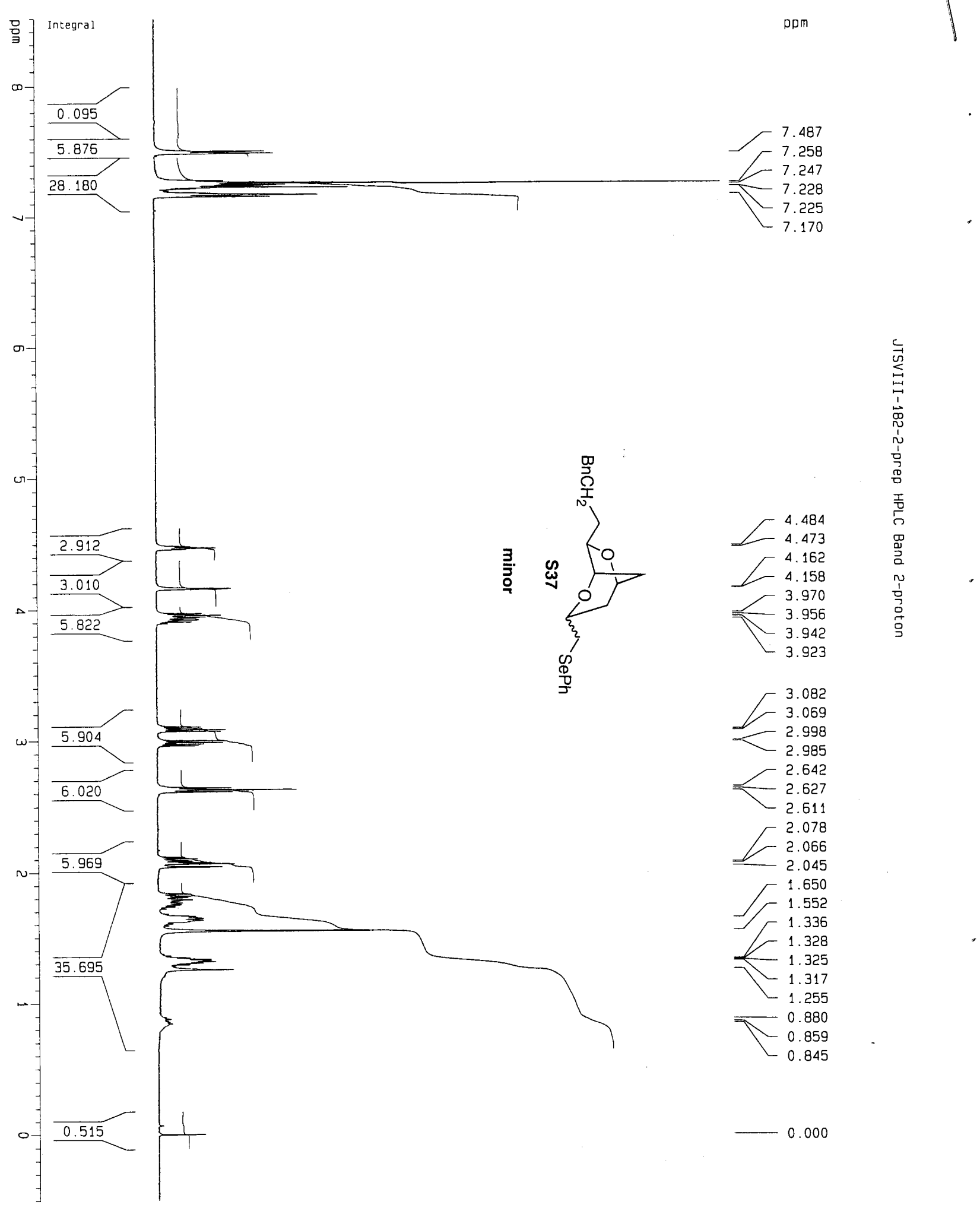



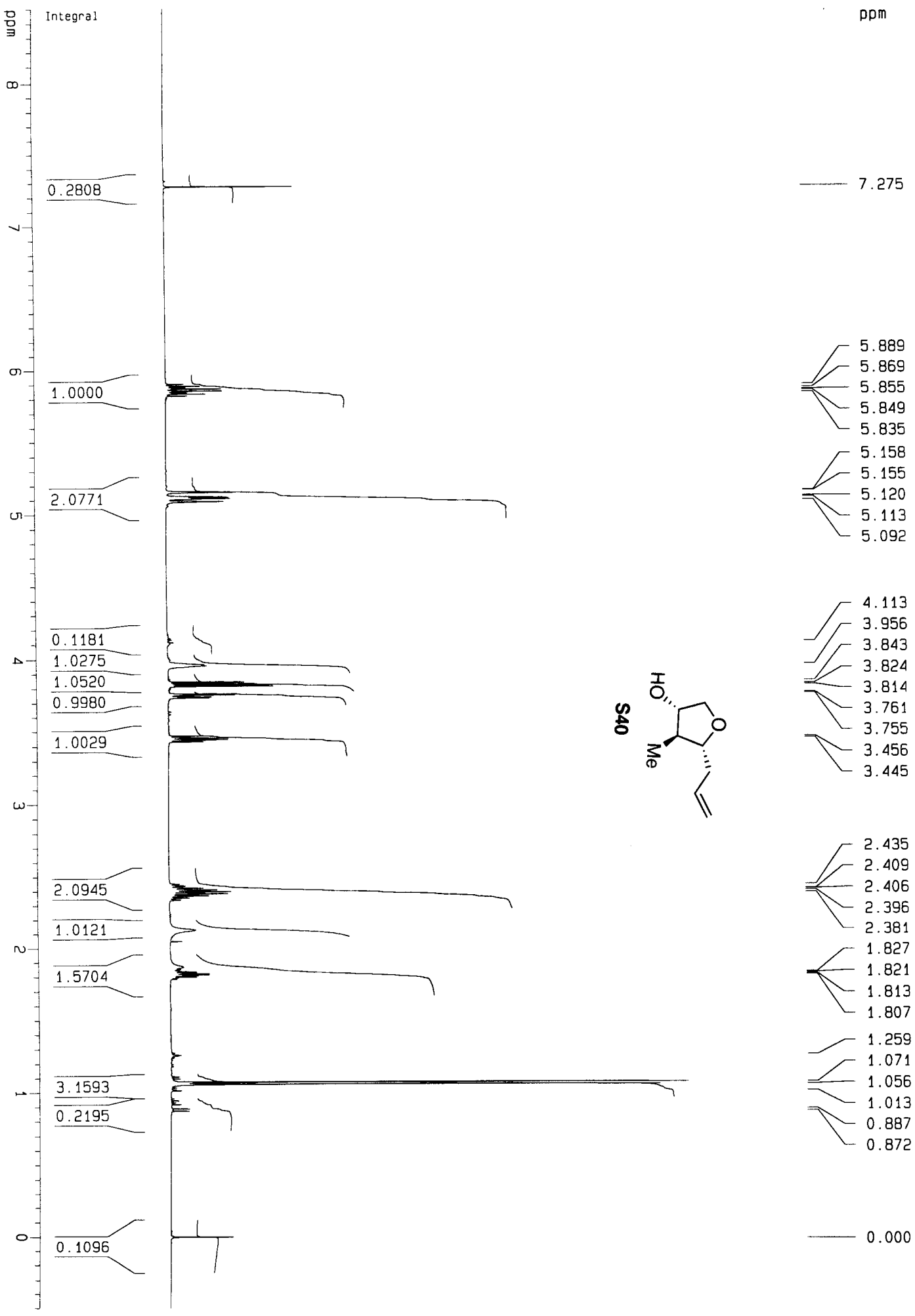

F 3.956

$-3.843$

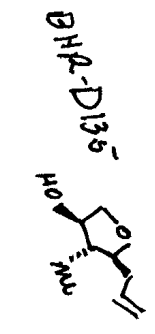

3.814
3.761

3.755

$\checkmark 3.456$

3.445

2.435

$-2.409$

2.406

2. 381

1.827

$\checkmark \begin{array}{r}1.821 \\ 1.813\end{array}$

ᄂ 1.807

1.259

$-1.071$

$-1.056$

$\sim 1.013$

$-0.887$

0.872

0.000 

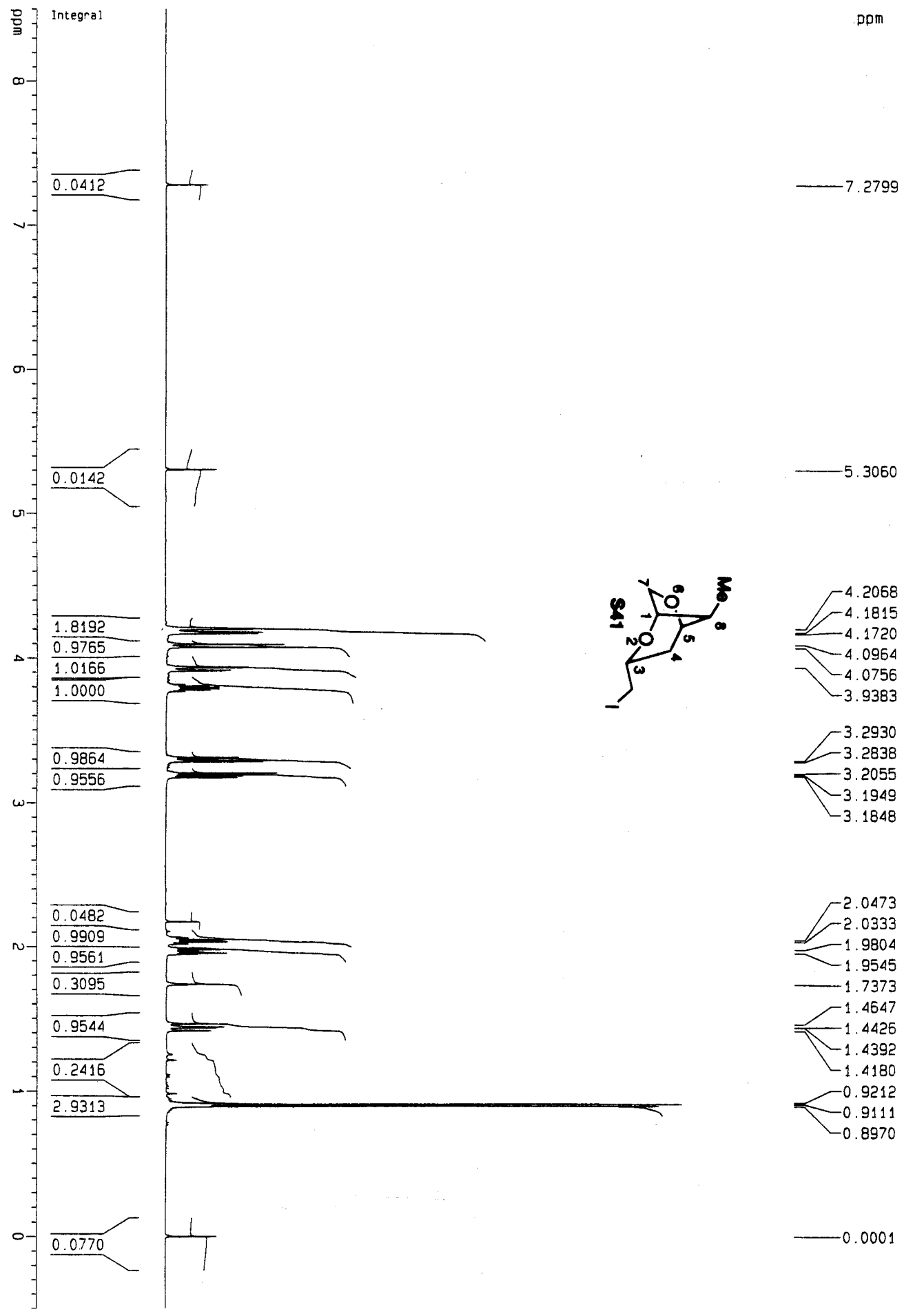

$-0.0001$ 

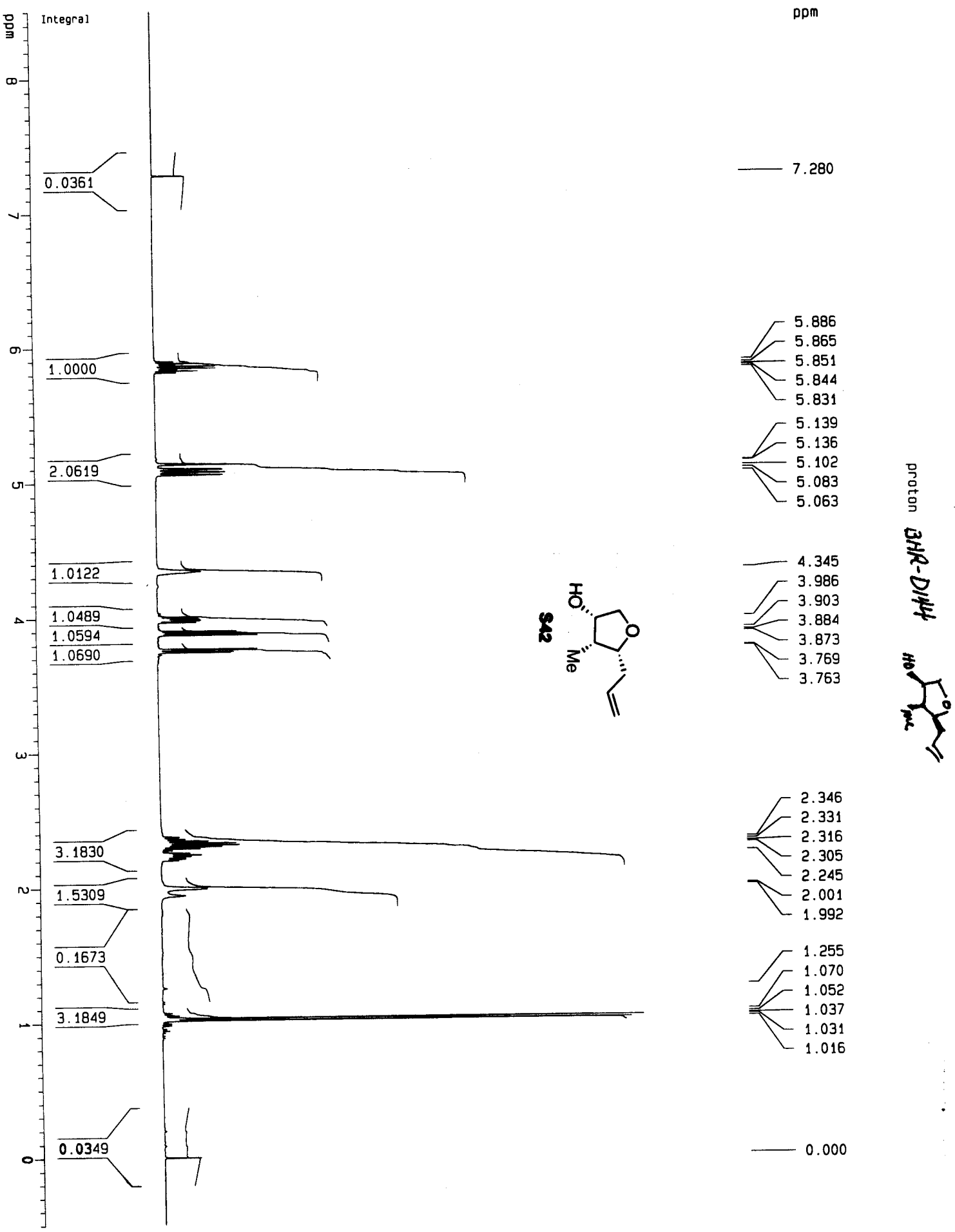


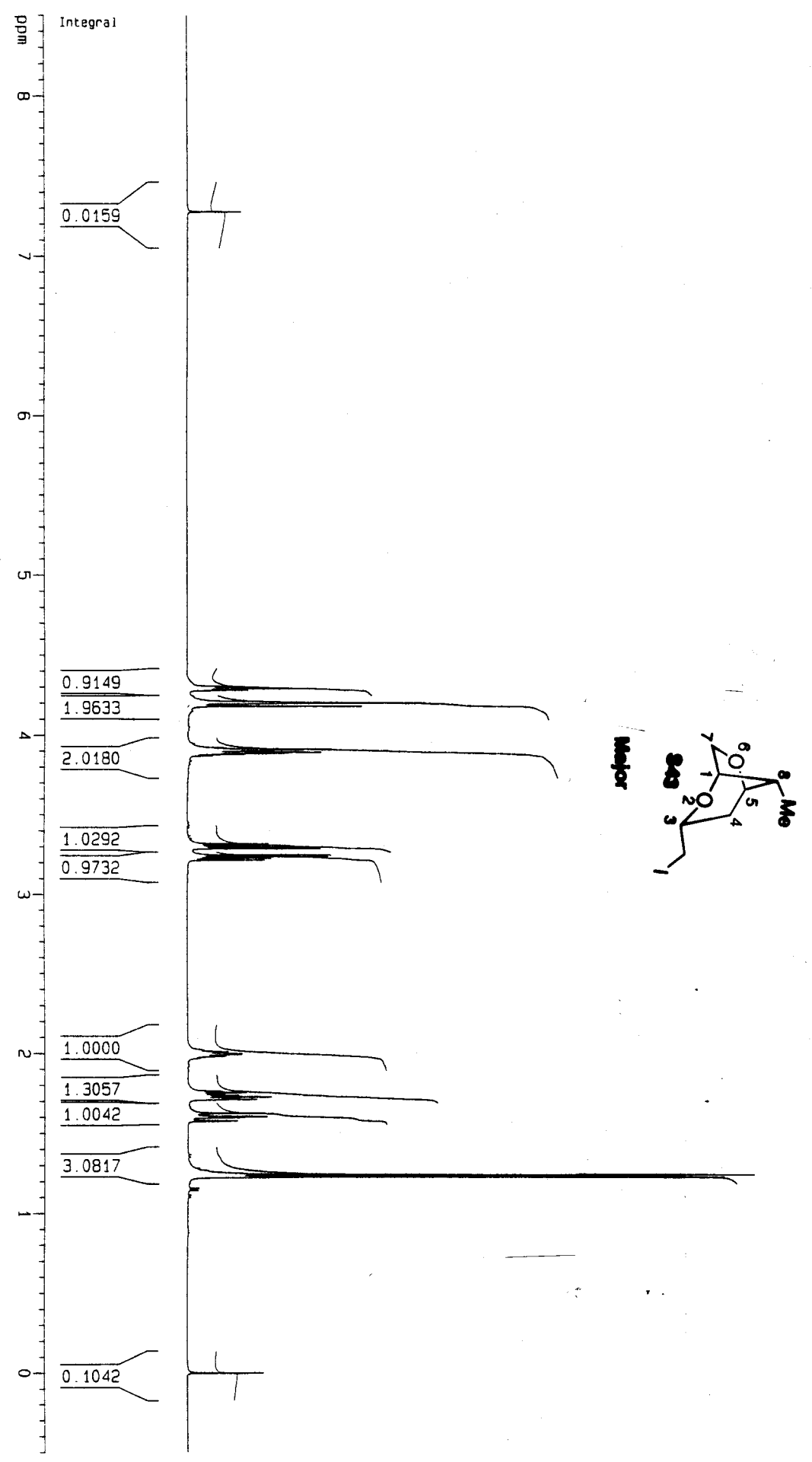

ppm
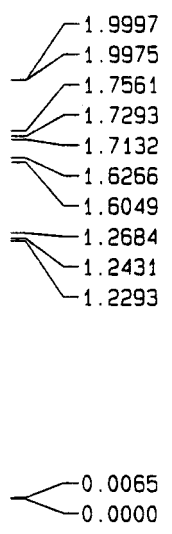